\title{
Stochastic variational formulas for solutions to linear diffusion equations
}

\author{
Joseph G. Conlon and Mohar Guha
}

\begin{abstract}
This paper is concerned with solutions to a one-dimensional linear diffusion equation and their relation to some problems in stochastic control theory. A stochastic variational formula is obtained for the logarithm of the solution to the diffusion equation, with terminal data which is the characteristic function of a set. In this case the terminal data for the control problem is singular, and hence standard theory does not apply. The variational formula is used to prove convergence, in the zero noise limit, of the cost function for the stochastic control problem and its first derivatives, to the corresponding quantities for a classical control problem.
\end{abstract}

\section{Introduction}

In this paper we shall be concerned with solutions to a linear diffusion equation and their relation to some problems in stochastic control theory. For $T>0$ let $b(y, t), y \in \mathbb{R}, t \leq T$, be a function with a partial derivative in $y$ that is continuous in $(y, t)$ and satisfies the uniform bound

$$
\sup \{|\partial b(y, t) / \partial y|: y \in \mathbb{R}, t \leq T\} \leq A,
$$

for some constant $A \geq 0$. We shall be interested in solutions $u_{\varepsilon}(x, y, t)$ to the partial differential equation (PDE)

$$
\frac{\partial u_{\varepsilon}}{\partial t}+b(y, t) \frac{\partial u_{\varepsilon}}{\partial y}+\frac{\varepsilon}{2} \frac{\partial^{2} u_{\varepsilon}}{\partial y^{2}}=0, \quad y \in \mathbb{R}, t<T,
$$

with terminal condition

$$
\begin{array}{ll}
\lim _{t \rightarrow T} u_{\varepsilon}(x, y, t)=0 & \text { for } y<x, \\
\lim _{t \rightarrow T} u_{\varepsilon}(x, y, t)=1 & \text { for } \quad y>x .
\end{array}
$$

Mathematics Subject Classification (2010): Primary 60J60, 93E20; Secondary 35K55. Keywords: Hamilton-Jacobi partial differential equation, stochastic control. 
It follows from standard methods [7] that $u_{\varepsilon}(x, y, t)$ is a continuous function of $(x, y, t)$ for $x, y \in \mathbb{R}, t<T$, and that also the first derivative $u_{\varepsilon}(x, y, t)$ in $t$ and second derivatives in $(x, y)$ exist and are continuous in $(x, y, t)$. Evidently $u_{\varepsilon}(x, y, t)$ is given in terms of the fundamental solution $G_{\varepsilon}\left(y, y^{\prime}, t, T\right)$ for (1.2) by the formula

$$
u_{\varepsilon}(x, y, t)=\int_{x}^{\infty} G_{\varepsilon}\left(y, y^{\prime}, t, T\right) d y^{\prime} .
$$

It is well known [12] that if $b(\cdot, \cdot)$ satisfies $(1.1)$ then the stochastic differential equation (SDE)

$$
d Y_{\varepsilon}(s)=b\left(Y_{\varepsilon}(s), s\right) d s+\sqrt{\varepsilon} d W(s),
$$

where $W(\cdot)$ is Brownian motion, has a unique solution in the interval $t \leq s \leq T$ with given initial condition $Y_{\varepsilon}(t)=y$. Furthermore, $u_{\varepsilon}(x, y, t)$ is related to solutions of (1.5) by the identity

$$
u_{\varepsilon}(x, y, t)=P\left(Y_{\varepsilon}(T)>x \mid Y_{\varepsilon}(t)=y\right), \quad t<T .
$$

The connection between solutions of (1.2)-(1.3) and control theory comes via the function $q_{\varepsilon}(x, y, t)$ defined by

$$
u_{\varepsilon}(x, y, t)=\exp \left[-q_{\varepsilon}(x, y, t) / \varepsilon\right] .
$$

In view of (1.6) the function $q_{\varepsilon}$ is positive, and by virtue of (1.2) and (1.3) it satisfies the PDE

$$
\frac{\partial q_{\varepsilon}}{\partial t}+b(y, t) \frac{\partial q_{\varepsilon}}{\partial y}-\frac{1}{2}\left(\frac{\partial q_{\varepsilon}}{\partial y}\right)^{2}+\frac{\varepsilon}{2} \frac{\partial^{2} q_{\varepsilon}}{\partial y^{2}}=0, \quad y \in \mathbb{R}, t<T,
$$

with terminal condition

$$
\begin{array}{ll}
\lim _{t \rightarrow T} q_{\varepsilon}(x, y, t)=\infty & \text { for } y<x, \\
\lim _{t \rightarrow T} q_{\varepsilon}(x, y, t)=0 & \text { for } y>x .
\end{array}
$$

If we let $\varepsilon \rightarrow 0$ in (1.8) we obtain a Hamilton-Jacobi equation, and therefore should expect that the limit of $q_{\varepsilon}(x, y, t)$ as $\varepsilon \rightarrow 0$ is given by the solution of a variational problem. This turns out to be the case. Let $q(x, y, t)$ be defined by

$$
q(x, y, t)=\min \left\{\frac{1}{2} \int_{t}^{T}\left[\frac{d y(s)}{d s}-b(y(s), s)\right]^{2} d s \mid y(t)=y, y(T)>x\right\}
$$

The functional in (1.10) is minimized over all paths $y(s), t \leq s \leq T$, with initial point $y(t)=y$ and terminal point $y(T)>x$. For $x \in \mathbb{R}$ and $t \leq T$, define the function $F(x, t)$ by $F(x, t)=y(t)$, where $y(\cdot)$ is the solution to the terminal value problem

$$
\frac{d y(s)}{d s}=b(y(s), s), \quad s \leq T, y(T)=x .
$$

Then one easily sees that $q(x, y, t)=0$ if $y \geq F(x, t)$, whence the function $q(x, y, t)$ is nontrivial only for sufficiently large negative values of $y$. In Section 3 we prove the following theorem showing that $q_{\varepsilon}$ converges to $q$ as $\varepsilon \rightarrow 0$. 
Theorem 1.1. Assume $b(\cdot, \cdot)$ satisfies (1.1). Then for $x, y \in \mathbb{R}, t<T$ and $0<\varepsilon<1$, there is a constant $C$ depending only on $x, y, t, T$ and $A$, such that

$$
\left|q_{\varepsilon}(x, y, t)-q(x, y, t)\right| \leq C \sqrt{\varepsilon}
$$

The inequality (1.12) implies, via (1.6), the large deviation result for solutions to the stochastic equation (1.5),

$$
\lim _{\varepsilon \rightarrow 0} \varepsilon \log \left[P\left(Y_{\varepsilon}(T)>x \mid Y_{\varepsilon}(t)=y\right)\right]=-q(x, y, t),
$$

a result which also follows from Theorem 1.2 of Chapter 4 of [8].

Inequalities of the type (1.12) for terminal data which is not singular, unlike in the case of (1.9), have been known for many years [2], [4], [5]. Theorem 5.1 of Crandall-Lions [2] implies that, in the case of constant $b(\cdot, \cdot)$, the inequality $(1.12)$ holds for $q_{\varepsilon}(x, y, t)$ satisfying (1.8) with terminal data $q_{\varepsilon}(x, \cdot, T)$ that is bounded and uniformly Lipschitz. A short elegant proof of the Crandall-Lions theorem has recently been given by Evans [3]. The techniques used in [2] and [3] are pure PDE methods. The approach of Fleming [4] is closer to the one we use in the present paper since it combines methods of stochastic analysis with PDE methods. However as in [2], terminal data is assumed to be Lipschitz, and his result applies for the PDE (1.8) only in the case when the function $b(\cdot, \cdot)$ is constant.

In proving Theorem 1.1 we take the approach of showing that in some sense $q_{\varepsilon}(x, y, t)$ is the cost function of a stochastic control problem. The formal limit as $\varepsilon \rightarrow 0$ of this stochastic control problem is a classical control problem with cost function $q(x, y, t)$ given by (1.10). The stochastic control problem can be described as follows: Let $y_{\varepsilon}(\cdot)$ be the solution to the stochastic differential equation,

$$
d y_{\varepsilon}(s)=\lambda_{\varepsilon}(\cdot, s) d s+\sqrt{\varepsilon} d W(s)
$$

where $\lambda_{\varepsilon}(\cdot, s)$ is a nonanticipating function. The cost function for the problem is given by the formula,

$$
q_{\varepsilon}(x, y, t)=\min _{\lambda_{\varepsilon}} E\left[\frac{1}{2} \int_{t}^{T}\left[\lambda_{\varepsilon}(\cdot, s)-b\left(y_{\varepsilon}(s), s\right)\right]^{2} d s \mid y_{\varepsilon}(t)=y, y_{\varepsilon}(T)>x\right] .
$$

Thus the minimum in (1.15) is to be taken over all nonanticipating $\lambda_{\varepsilon}(\cdot, s), t \leq$ $s<T$, which have the property that the solutions of (1.14) with initial condition $y_{\varepsilon}(t)=y$ satisfy the terminal condition $y_{\varepsilon}(T)>x$ with probability 1 . One expects that the function $q_{\varepsilon}(x, y, t)$ of $(1.15)$ is identical to the function $q_{\varepsilon}(x, y, t)$ of (1.7), and the optimal controller $\lambda_{\varepsilon}(\cdot, s)$ is given by the formula

$$
\lambda_{\varepsilon}(\cdot, s)=\lambda_{\varepsilon}^{*}\left(x, y_{\varepsilon}(s), s\right)=b\left(y_{\varepsilon}(s), s\right)-\frac{\partial q_{\varepsilon}}{\partial y}\left(x, y_{\varepsilon}(s), s\right)
$$

This is not so easy to prove. An immediate question that arises is how to define a suitable space of nonanticipating functions $\lambda_{\varepsilon}(\cdot, s), t \leq s<T$, which have the property that solutions of (1.14) with initial condition $y_{\varepsilon}(t)=y$ satisfy $y_{\varepsilon}(T)>x$ with probability 1 . 
For the purposes of proving Theorem 1.1, it is actually only necessary to show that equality in (1.15) holds in the approximate sense

$$
q_{\varepsilon}(x, y, t)=E\left[\frac{1}{2} \int_{t}^{T-\sqrt{\varepsilon}}\left[\lambda_{\varepsilon}(\cdot, s)-b\left(y_{\varepsilon}(s), s\right)\right]^{2} d s \mid y_{\varepsilon}(t)=y\right]+O(\sqrt{\varepsilon}),
$$

where $\lambda_{\varepsilon}(\cdot, s)$ is a controller chosen suitably close to the formal optimizer (1.16). The identity (1.17) turns out to be much easier to establish than the equality in (1.15) when $\lambda_{\varepsilon}(\cdot, s), t \leq s<T$, is given by (1.16). The reason is that the function $q_{\varepsilon}(x, \cdot, T-\sqrt{\varepsilon})$ can be shown to have good Lipschitz properties by using PDE methods. The original stochastic control problem on the time interval $t<s<T$ is then replaced by a stochastic control problem on the interval $t<s<T-\sqrt{\varepsilon}$, and hence becomes closer to the situation studied in [2], [3], [4]. In Section 3 we obtain upper and lower bounds on $q_{\varepsilon}(x, y, t)$ of the form $q(x, y, t)+O(\sqrt{\varepsilon})$ by exploiting the variational formulation of the stochastic control problem on the interval $t<s<T-\sqrt{\varepsilon}$. To get the upper bound for $q_{\varepsilon}(x, y, t)$ we choose $\lambda_{\varepsilon}(\cdot, s)$ to be the optimal controller for the corresponding classical control problem (1.10). To get the lower bound on $q_{\varepsilon}(x, y, t)$ we choose $\lambda_{\varepsilon}(\cdot, s)$ to be given by $(1.16)$.

In Section 4 and Section 6 we address the issue of understanding in what sense (1.15) holds when $q_{\varepsilon}(x, y, t)$ is given by (1.7). Instead of attempting to establish the formula (1.15) we shall confine ourselves to the simpler problem of showing that the expectation on the right-hand side of (1.15) is greater than or equal to $q_{\varepsilon}(x, y, t)$ for certain nonanticipating functions $\lambda_{\varepsilon}(\cdot, s), t \leq s<T$, and that there is equality when the controller $\lambda_{\varepsilon}$ is taken to be the formal optimal controller $\lambda_{\varepsilon}^{*}$ of (1.16).

In Section 4 we show that the solution $y_{\varepsilon}(s), t \leq s<T$, of (1.14) with initial condition $y_{\varepsilon}(t)=y$ and $\lambda_{\varepsilon}(\cdot, s)$ given by the optimal controller (1.16), has the property that

$$
\liminf _{t \rightarrow T} y_{\varepsilon}(t)>x \quad \text { with probability } 1 .
$$

The proof of (1.18) depends crucially on obtaining a lower bound on the derivative of the function $q_{\varepsilon}$ of $(1.7)$,

$$
-\frac{\partial q_{\varepsilon}}{\partial y}(x, y, t) \geq \frac{x-y}{T-t}[1-\eta(\delta)], \quad 0<T-t<\delta, x-y<\gamma,
$$

where $\gamma$ is independent of $\delta$ and $\lim _{\delta \rightarrow 0} \eta(\delta)=0$. Observe that the inequality (1.19) is only nontrivial for $y<x$ since $-\partial q_{\varepsilon}(x, y, t) / \partial y \geq 0, y \in \mathbb{R}$, by the maximum principle. The proof of (1.19) relies on the use of the Cameron-Martin formula [21] applied to the diffusion $Y_{\varepsilon}(\cdot)$ of (1.5). One can see from (1.4) that the inequality (1.19) gives some information about the short time asymptotics of fundamental solutions to diffusion equations. Over several decades there has been much research [11], [17], [18], [22] devoted to this subject. In particular, Molchanov [18] has obtained short time asymptotic formulas for diffusions with bounded drift. These results have been used by Fleming and Sheu [6] to prove a representation formula analogous to (1.15) for the logarithm of the fundamental solution. 
In order to establish that the expectation on the right-hand side of (1.15) with $\lambda_{\varepsilon}(\cdot, s), t \leq s<T$, given by (1.16) is equal to the left-hand side, one needs to prove that the inequality (1.19) holds uniformly for $y \in \mathbb{R}$, i.e., $\gamma=\infty$. This turns out to be a considerably more difficult task than proving (1.19) for some $\gamma>0$. It is not possible to obtain estimates by means of the Cameron-Martin formula, and instead one uses an induction argument. The problem of obtaining a uniform lower bound (1.19) is closely related to the problem of estimating probabilities for the diffusion $Y_{\varepsilon}(\cdot)$ of $(1.5)$ tied at 2 different times. In Section 5 we prove the following:

Theorem 1.2. Suppose $b(\cdot, s), 0 \leq s \leq T$, satisfies (1.1) and suppose, in addition, $b(0, s)=0,0 \leq s \leq T$. Then there exist positive universal constants $\eta, C_{1}, C_{2}, \gamma_{1}$ and $\gamma_{2}$, such that

$$
\begin{aligned}
& P\left(Y_{\varepsilon}(t)<\frac{C_{1}(T-t) y}{T} \mid Y_{\varepsilon}(0)=y, Y_{\varepsilon}(T)=0\right) \\
& \leq \exp \left[-\frac{\gamma_{1}(T-t) y^{2}}{\varepsilon T^{2}}\right], \quad y<-T \sqrt{\varepsilon /(T-t)}, \\
& \begin{aligned}
P\left(Y_{\varepsilon}(t)>\frac{C_{2}(T-t) y}{T} \mid Y_{\varepsilon}(0)=y, Y_{\varepsilon}(T)=0\right) \\
\leq \exp \left[-\frac{\gamma_{2}(T-t) y^{2}}{\varepsilon T^{2}}\right], \quad y<-T \sqrt{\varepsilon /(T-t)},
\end{aligned}
\end{aligned}
$$

provided $A T<\eta$ and $T-t<T / 2$.

In Section 6 we not only show that the expectation on the right-hand side of $(1.15)$ with $\lambda_{\varepsilon}(\cdot, s)$ given by $(1.16)$ equals the left-hand side. We also obtain corresponding formulas for the first derivatives of $q_{\varepsilon}(x, y, t)$ in $x$ and $y$. An immediate consequence of this, Corollary 6.3 , is that the fundamental solution $G_{\varepsilon}$ for (1.2) satisfies the inequality

$$
G_{\varepsilon}(y, x, t, T) \leq[1+(T-t) A] u_{\varepsilon}(x, y, t)\left[\frac{-2 \log u_{\varepsilon}(x, y, t)}{\varepsilon(T-t)}\right]^{1 / 2},
$$

where $A$ is the constant in (1.1) and $u_{\varepsilon}(x, y, t)$ is given by (1.4). The inequality (1.22) appears to be nontrivial even in the case $b \equiv 0$, where it states that the cumulative distribution function $N(\cdot)$ for the standard normal variable,

$$
N(z)=\frac{1}{\sqrt{2 \pi}} \int_{-\infty}^{z} \exp \left(-\rho^{2} / 2\right) d \rho=\frac{1}{2}+\frac{1}{2} \operatorname{sign}(z) \operatorname{erf}\left(\frac{|z|}{\sqrt{2}}\right),
$$

satisfies the inequality

$$
\exp \left(-z^{2} / 2\right) \leq 2 \sqrt{\pi} N(z)[-\log N(z)]^{1 / 2}, \quad z \in \mathbb{R} .
$$

Let us assume now that the function $b(y, t)$, in addition to satisfying (1.1), is also concave in $y$ for each $t \leq T$. In Section 2 we show that in this case the function 
$q(x, y, t)$ of $(1.10)$ is $C^{1}$ in $(x, y, t)$ and is a classical solution of the $\varepsilon=0$ HamiltonJacobi equation (1.8). Furthermore, for any $t<T$ the function $q(x, y, t)$ is convex in $(x, y)$ and its second derivatives in $(x, y)$ exist and are continuous on the set $\{(x, y, t): x, y \in \mathbb{R}, t<T, y \neq F(x, t)\}$, where $F(x, t)$ is the function defined by (1.11). In the appendix we prove, using the method of Korevaar [9], [10], [14], that the function $q_{\varepsilon}(x, y, t)$ defined by $(1.7)$ is also convex in $(x, y)$ for any $t<T$. Although Korevaar's method is simple in concept, considerable difficulty arises here in its implementation due to the fact that we need to approximate solutions of the linear equation (1.2) by solutions of a quasi-linear equation (A.34). Hence we need regularity theory, Proposition A.4, for solutions to quasi-linear equations [7], [15]. Alternative approaches [1], [16] to Korevaar's method seem to also give rise to comparable technical difficulties in the implementation.

The proof that for fixed $(x, t)$ the function $q_{\varepsilon}(x, y, t)$ is convex in $y$, Theorem A.2, is much easier to establish than the joint convexity in $(x, y)$. Using this fact and the representation theorem of Section 6 we prove in Section 7 convergence of first derivatives of $q_{\varepsilon}(x, y, t)$ in $(x, y)$ to first derivatives of $q(x, y, t)$ as $\varepsilon \rightarrow 0$.

Theorem 1.3. Assume $b(\cdot, \cdot)$ satisfies (1.1) and in addition that $b(y, t)$ is concave in $y$ for each $t \leq T$. Then $q(x, y, t)$ is $C^{1}$ in $(x, y, t)$ for $t<T$ and

$$
\begin{aligned}
& \lim _{\varepsilon \rightarrow 0} \frac{\partial q_{\varepsilon}}{\partial x}(x, y, t)=\frac{\partial q}{\partial x}(x, y, t), \quad x, y \in \mathbb{R}, t<T, \\
& \lim _{\varepsilon \rightarrow 0} \frac{\partial q_{\varepsilon}}{\partial y}(x, y, t)=\frac{\partial q}{\partial y}(x, y, t), \quad x, y \in \mathbb{R}, t<T .
\end{aligned}
$$

Theorem 1.3 gives no rate of convergence as $\varepsilon \rightarrow 0$ like in Theorem 1.1, but if one assumes some Hölder continuity of $\partial b(y, t) / \partial y$ in $y$, then the proof of the theorem yields a rate of convergence which is a power of $\varepsilon$. It is of some interest to compare Theorem 1.3 to the results of Kifer [13] on the asymptotics of the fundamental solution $G_{\varepsilon}(y, x, t, T)$ defined by (1.4) as $\varepsilon \rightarrow 0$. In that paper asymptotic formulas are established by using the fact that $G_{\varepsilon}(y, \cdot, t, T)$ is the probability density function for the random variable $Y_{\varepsilon}(T)$ conditioned on $Y_{\varepsilon}(t)=y$. Estimates on the probability density are then obtained by using large deviation techniques [8]. Emphasis in the paper is placed on the local nature of the result. Thus the behavior of the drift $b(\cdot, \cdot)$ far from the minimizing trajectory in $(1.10)$ is shown to be largely irrelevant.

\section{A classical control problem}

Let $b(y, s), y \in \mathbb{R}$, and $s \leq T$ satisfy (1.1) and consider the control dynamics

$$
\frac{d y}{d s}=\lambda(s), \quad t \leq s \leq T, \quad y(t)=y
$$

where the controller $\lambda(s), t \leq s \leq T$, is assumed to be piecewise continuous. 
We shall be interested in the optimal control problem with cost function $q(x, y, t)$, $x, y \in \mathbb{R}, t<T$, defined by

$$
q(x, y, t)=\min _{\lambda(\cdot)}\left\{\frac{1}{2} \int_{t}^{T}[\lambda(s)-b(y(s), s)]^{2} d s \mid y(t)=y, y(T)>x\right\} .
$$

Formally the function $q(x, y, t)$ of $(2.2)$ satisfies the Hamilton-Jacobi equation

$$
\frac{\partial q}{\partial t}+b(y, t) \frac{\partial q}{\partial y}-\frac{1}{2}\left(\frac{\partial q}{\partial y}\right)^{2}=0 \text {. }
$$

Since the minimum in (2.2) is over paths $y(s), t \leq s \leq T$, satisfying $y(T)>x$, the terminal condition on the PDE (2.3) is given by

$$
\begin{aligned}
& \lim _{t \rightarrow T} q(x, y, t)=\infty, \quad y<x, \\
& \lim _{t \rightarrow T} q(x, y, t)=0, \quad y>x .
\end{aligned}
$$

The optimal controller $\lambda(s)$ for $(2.2)$ is given by the formula

$$
\lambda(s)=\lambda^{*}(x, y(s), s)=b(y(s), s)-\partial q(x, y(s), s) / \partial y, \quad t \leq s \leq T,
$$

and the Euler-Lagrange equation for the minimizing trajectory is given by

$$
\frac{d}{d s}\left[\frac{d y}{d s}-b(y(s), s)\right]+\frac{\partial b}{\partial y}(y(s), s)\left[\frac{d y}{d s}-b(y(s), s)\right]=0, \quad t \leq s \leq T .
$$

Our first goal is to prove that there exists a minimizer for the variational problem. We have already observed that if $F(\cdot, \cdot)$ is the function defined by (1.11), then $q(x, y, t)=0$ if $y \geq F(x, t)$. Evidently in this case there is a unique minimizer $y(\cdot)$ for $(2.2)$, which is the solution to the differential equation (1.11) with initial condition $y(t)=y$. For $y<F(x, t)$ we need to define a space of functions $y(s), t \leq s \leq T$, over which to minimize the expression in (2.2). For any $f \in L^{2}[t, T]$, let $y(\cdot)$ be determined from $f$ by

$$
y(s)=y+\int_{t}^{s} f\left(s^{\prime}\right) d s^{\prime} .
$$

Thus $y(\cdot)$ is Hölder continuous of order $1 / 2$ on $[t, T]$ and $y(t)=y$. We define $E_{x, y, t}$ to be the space of all such functions $y(s), t \leq s \leq T$, with $f \in L^{2}[t, T]$ and $y(T) \geq x$. The distance between two functions $y_{1}, y_{2} \in E_{x, y, t}$ is given by the norm $\left\|y_{1}-y_{2}\right\|=\left\|f_{1}-f_{2}\right\|_{2}$, where $y_{1}$ corresponds to $f_{1}$ and $y_{2}$ to $f_{2}$ in (2.7). Evidently the space $E_{x, y, t}$ is complete under this distance function. Now (2.6) indicates that on a minimizer $y(s), t \leq s \leq T$, for $(2.2)$ the expression $y^{\prime}(s)-b(y(s), s)$ does not change sign for $s$ in the interval $[t, T]$. We shall show that if $y<F(x, t)$ the sign is in fact positive.

Proposition 2.1. Assume the function $b(\cdot, \cdot)$ satisfies (1.1). Then there exists a minimizer $y(\cdot) \in E_{x, y, t}$ of the variational problem (2.2). Any minimizer $y(\cdot)$ has the property that $y(\cdot)$ is $C^{1}$ in $[t, T]$. If $y<F(x, t)$ then $y^{\prime}(s)>b(y(s), s)$, for $t \leq s \leq T$, and $y(T)=x$. The function $q(x, y, t)$ of $(2.2)$ is continuous for $(x, y) \in \mathbb{R}^{2}$, and $t<T$. 
Proof. We define a functional $\mathcal{F}[y(\cdot)]$ on $E_{x, y, t}$ by

$$
\mathcal{F}[y(\cdot)]=\frac{1}{2} \int_{t}^{T}\left[\frac{d y}{d s}-b(y(s), s)\right]^{2} d s .
$$

Following the standard method $[20]$ we show that $\mathcal{F}[\cdot]$ is weakly lower semicontinuous on $E_{x, y, t}$. To this end let $y_{N}(\cdot), N \geq 1$, be a sequence in $E_{x, y, t}$ converging weakly to $y_{\infty}(\cdot) \in E_{x, y, t}$. Hence if $f_{N}, N \geq 1, f_{\infty}$ in $L^{2}[t, T]$ are associated with $y_{N}(\cdot), N \geq 1$, and $y_{\infty}(\cdot)$ respectively, we have that

$$
\lim _{N \rightarrow \infty}\left\langle f, f_{N}\right\rangle=\left\langle f, f_{\infty}\right\rangle, \quad f \in L^{2}[t, T] .
$$

From the uniform boundedness principle [20] it follows that $\sup _{N>1}\left\|f_{N}\right\|_{2}<\infty$. It also follows from (2.9) that $\lim _{N \rightarrow \infty} y_{N}(s)=y_{\infty}(s), t \leq s \leq T$, and that $\sup \left\{\left|y_{N}(s)\right|: N \geq 1, t \leq s \leq T\right\}<\infty$. Hence by the dominated convergence theorem one has that

$$
\lim _{N \rightarrow \infty} \int_{t}^{T} b\left(y_{N}(s), s\right)^{2} d s=\int_{t}^{T} b\left(y_{\infty}(s), s\right)^{2} d s .
$$

Using the uniform boundedness of the $f_{N}, N \geq 1$, we also have that

$$
\lim _{N \rightarrow \infty} \int_{t}^{T}\left[b\left(y_{N}(s), s\right)-b\left(y_{\infty}(s), s\right)\right] f_{N}(s) d s=0 .
$$

Hence using (2.9) again we conclude that

$$
\lim _{N \rightarrow \infty} \int_{t}^{T} b\left(y_{N}(s), s\right) f_{N}(s) d s=\int_{t}^{T} b\left(y_{\infty}(s), s\right) f_{\infty}(s) d s .
$$

Now (2.10) and (2.11) imply that

$$
\begin{aligned}
\liminf _{N \rightarrow \infty} \mathcal{F}\left[y_{N}(\cdot)\right]= & \frac{1}{2} \liminf _{N \rightarrow \infty} \int_{t}^{T}\left[\frac{d y_{N}(s)}{d s}\right]^{2} d s \\
& -\int_{t}^{T} b\left(y_{\infty}(s), s\right) \frac{d y_{\infty}(s)}{d s} d s+\frac{1}{2} \int_{t}^{T} b\left(y_{\infty}(s), s\right)^{2} d s .
\end{aligned}
$$

The lower semicontinuity of $\mathcal{F}[\cdot]$ on $E_{x, y, t}$ follows from the inequality

$$
\frac{1}{2} \int_{t}^{T}\left[\frac{d y_{\infty}(s)}{d s}\right]^{2} d s \leq \frac{1}{2} \liminf _{N \rightarrow \infty} \int_{t}^{T}\left[\frac{d y_{N}(s)}{d s}\right]^{2} d s
$$

which is a consequence of the convexity of the Dirichlet form [20]. One easily concludes from the lower semicontinuity of $\mathcal{F}[\cdot]$ the existence of a minimizer $y(\cdot) \in E_{x, y, t}$.

Suppose now $y(\cdot) \in E_{x, y, t}$ is a minimizer for $\mathcal{F}[\cdot]$. Then the first variation of $\mathcal{F}[\cdot]$ about $y(\cdot)$ must be 0 , whence

$$
\int_{t}^{T}\left[\frac{d \varphi(s)}{d s}-\frac{\partial b}{\partial y}(y(s), s) \varphi(s)\right]\left[\frac{d y(s)}{d s}-b(y(s), s)\right] d s=0
$$


provided $\varphi(\cdot)$ is a $C^{1}$ function satisfying $\varphi(t)=0$ and $\varphi(T)=0$. Setting

$$
\varphi(s)=\psi(s) \exp \left[\int_{t}^{s} \frac{\partial b}{\partial y}\left(y\left(s^{\prime}\right), s^{\prime}\right) d s^{\prime}\right]=\psi(s) V(s),
$$

it follows from (2.12) that

$$
\int_{t}^{T} \frac{d \psi}{d s}\left[\frac{d y}{d s}-b(y(s), s)\right] V(s) d s=0,
$$

for all $C^{1}$ functions $\psi:[t, T] \rightarrow \mathbb{R}$ with $\psi(t)=\psi(T)=0$. Equation (2.13) implies that

$$
\left[\frac{d y}{d s}-b(y(s), s)\right] V(s)=\text { constant }, \quad t \leq s \leq T
$$

from which we can conclude that if $y<F(x, t)$ then $y^{\prime}(s)>b(y(s), s)$ for all $s$, $t \leq s \leq T$, and $y(\cdot)$ is $C^{1}$. It also follows that $y(T)=x$, for if $y(T)>x$ then there exists $t_{1}<T$ such that if $y_{1}(s), t_{1} \leq s \leq T$, satisfies $y_{1}\left(t_{1}\right)=y\left(t_{1}\right)$ and $y_{1}^{\prime}(s)=$ $b\left(y_{1}(s), s\right), t_{1} \leq s \leq T$, then $y_{1}(T)>x$. Evidently the function $y^{*}(s), t \leq s \leq T$, defined by $y^{*}(s)=y(s), t \leq s \leq t_{1}$ and $y^{*}(s)=y_{1}(s), t_{1} \leq s \leq T$, is in $E_{x, y, t}$ and satisfies $\mathcal{F}\left[y^{*}(\cdot)\right]<\mathcal{F}[y(\cdot)]$, yielding a contradiction. One can argue in a similar way to prove the continuity of the function $q(x, y, t),(x, y) \in \mathbb{R}^{2}, t<T$.

We have already observed that for $y \geq F(x, t)$ there is a unique minimizer $y(\cdot) \in E_{x, y, t}$ for the variational expression (2.2) and it is given by the solution $y(\cdot)$ of equation (1.11) with initial condition $y(t)=y$. For $y<F(x, t)$ we need to impose some condition on the function $b(\cdot, \cdot)$ beyond $(1.1)$ to guarantee a unique minimizer. To see what such a condition should be, suppose that $y(s), t \leq s \leq T$, is a solution of the Euler-Lagrange equation (2.6) with initial conditions satisfying

$$
y(t)=y, \quad y^{\prime}(t)>b(y, t) .
$$

Hence (2.6) implies that $y^{\prime}(s)>b(y(s), s), t \leq s \leq T$. Suppose now that $y(s)+\varphi(s), t \leq s \leq T$, is also a solution to (2.6) with $\varphi(t)=0$ and $\varphi^{\prime}(t)=\varepsilon$. Then, to first order in $\varepsilon$, the function $\varphi(s), t \leq s \leq T$, satisfies the linear equation

$$
\begin{aligned}
\frac{d^{2} \varphi}{d s^{2}} & -\frac{d}{d s}\left[\frac{\partial b}{\partial y}(y(s), s) \varphi(s)\right]+\frac{\partial b}{\partial y}(y(s), s) \frac{d \varphi(s)}{d s} \\
& -\left[\frac{\partial b}{\partial y}(y(s), s)\right]^{2} \varphi(s)+\frac{\partial^{2} b}{\partial y^{2}}(y(s), s)\left[\frac{d y}{d s}-b(y(s), s)\right] \varphi(s)=0 .
\end{aligned}
$$

Suppose now that $\varphi(\tau)=0$ for some $\tau, t<\tau \leq T$. Then, on multiplying (2.16) by $\varphi(s)$ and integrating over the interval $t \leq s \leq \tau$, we get

$$
\begin{aligned}
-\int_{t}^{\tau}\left[\frac{d \varphi(s)}{d s}\right]^{2} d s & +2 \int_{t}^{\tau} \frac{\partial b}{\partial y}(y(s), s) \varphi(s) \frac{d \varphi(s)}{d s} d s \\
& -\int_{t}^{\tau}\left[\frac{\partial b}{\partial y}(y(s), s)\right]^{2} \varphi(s)^{2} d s-\int_{t}^{\tau} V(s) \varphi(s)^{2} d s=0,
\end{aligned}
$$


where $V(s)$ is given by the formula

$$
V(s)=-\frac{\partial^{2} b}{\partial y^{2}}(y(s), s)\left[\frac{d y}{d s}-b(y(s), s)\right] .
$$

Observe that by the Schwarz inequality we have

$$
2 \int_{t}^{\tau} \frac{\partial b}{\partial y}(y(s), s) \varphi(s) \frac{d \varphi(s)}{d s} d s \leq \int_{t}^{\tau}\left(\frac{d \varphi(s)}{d s}\right)^{2}+\int_{t}^{\tau}\left[\frac{\partial b}{\partial y}(y(s), s)\right]^{2} \varphi(s)^{2} d s,
$$

with strict inequality in general. Thus if $V(\cdot)$ in $(2.18)$ is nonnegative the expression (2.17) is strictly negative in general. Since $V(\cdot)$ is nonnegative if the function $b(y, s)$ is concave in $y$, it appears that one gets a contradiction to the fact that $(2.17)$ is zero when one assumes that $b(y, s)$ is concave in $y, t \leq s \leq T$. We conclude therefore that the trajectories $y(\cdot)$ of the Euler-Lagrange equation (2.6) which satisfy (2.15) are nonintersecting. In particular, for $y<F(x, t)$ there is exactly one which has the property that $y(t)=y$ and $y(T)=x$. We make this argument rigorous in the following proposition.

Proposition 2.2. Assume the function $b(\cdot, \cdot)$ satisfies (1.1) and that $b(y, s)$ is concave in $y$ for $y \in \mathbb{R}$ and $s \in[t, T]$. Then the minimizer $y(\cdot) \in E_{x, y, t}$ of the variational problem (2.2) is unique for all $(x, y) \in \mathbb{R}^{2}$. Furthermore the function $q(x, y, t)$ of $(2.2)$ is $C^{1}$ for $(x, y) \in \mathbb{R}^{2}$ and $t<T$.

Proof. Since the minimizer is clearly unique for $y \geq F(x, t)$ we assume $y<F(x, t)$. We show that the functional $\mathcal{F}[\cdot]$ of $(2.8)$ has a convexity property provided $b(y, s)$ is concave in $y, t \leq s \leq T$. Let $E$ be the set of $C^{1}$ functions $y(\cdot)$ on $[t, T]$ which satisfy $y^{\prime}(s) \geq b(y(s), s), t \leq s \leq T$. It is evident that $E$ is convex in $y(\cdot)$ for $t \leq s \leq T$, in the sense that

$$
y_{1}(\cdot), \quad y_{2}(\cdot), \quad \lambda y_{1}(\cdot)+(1-\lambda) y_{2}(\cdot) \in E, \quad 0 \leq \lambda \leq 1,
$$

implies

$$
\mathcal{F}\left[\lambda y_{1}(\cdot)+(1-\lambda) y_{2}(\cdot)\right] \leq \lambda \mathcal{F}\left[y_{1}(\cdot)\right]+(1-\lambda) \mathcal{F}\left[y_{2}(\cdot)\right]
$$

To prove (2.19) we write

$$
\begin{aligned}
\mathcal{F}[\lambda & \left.y_{1}(\cdot)+(1-\lambda) y_{2}(\cdot)\right] \\
= & \frac{1}{2} \int_{t}^{T}\left[\lambda\left\{\frac{d y_{1}}{d s}-b\left(y_{1}(s), s\right)\right\}+(1-\lambda)\left\{\frac{d y_{2}}{d s}-b\left(y_{2}(s), s\right)\right\}\right. \\
& \left.-\left\{b\left(\lambda y_{1}(s)+(1-\lambda) y_{2}(s), s\right)-\lambda b\left(y_{1}(s), s\right)-(1-\lambda) b\left(y_{2}(s), s\right)\right\}\right]^{2} d s .
\end{aligned}
$$

Since $y_{1}(\cdot), y_{2}(\cdot) \in E$ and $b(y, s)$ is concave in $y, t \leq s \leq T$, each term in the last expression inside curly braces is nonnegative. Assuming also that $\lambda y_{1}(\cdot)+$ $(1-\lambda) y_{2}(\cdot) \in E$, we have that

$$
\begin{aligned}
0 & \leq b\left(\lambda y_{1}(s)+(1-\lambda) y_{2}(s), s\right)-\lambda b\left(y_{1}(s), s\right)-(1-\lambda) b\left(y_{2}(s), s\right) \\
& \leq 2\left[\lambda\left\{\frac{d y_{1}}{d s}-b\left(y_{1}(s), s\right)\right\}+(1-\lambda)\left\{\frac{d y_{2}}{d s}-b\left(y_{2}(s), s\right)\right\}\right], \quad t \leq s \leq T .
\end{aligned}
$$


We conclude therefore that

$$
\begin{aligned}
\mathcal{F}\left[\lambda y_{1}(\cdot)\right. & \left.+(1-\lambda) y_{2}(\cdot)\right] \\
& \leq \frac{1}{2} \int_{t}^{T}\left[\lambda\left\{\frac{d y_{1}}{d s}-b(y(s), s)\right\}+(1-\lambda)\left\{\frac{d y_{2}}{d s}-b(y(s), s)\right\}\right]^{2} d s \\
& \leq \lambda \mathcal{F}\left[y_{1}(\cdot)\right]+(1-\lambda) \mathcal{F}\left[y_{2}(\cdot)\right],
\end{aligned}
$$

and hence (2.19) holds.

The uniqueness of the minimizer $y(\cdot) \in E_{x, y, t}$ follows from the strict convexity of $\mathcal{F}[\cdot]$ in the sense of (2.19). Let us assume $y_{1}(\cdot), y_{2}(\cdot) \in E_{x, y, t}$ are two minimizers where $y<F(x, t)$. Then by Proposition 2.1 the functions $y_{1}(\cdot)$ and $y_{2}(\cdot)$ are in the set $E$ and for sufficiently small $\lambda>0$ the function $\lambda y_{1}(\cdot)+(1-\lambda) y_{2}(\cdot)$ is also in $E$, whence $(2.19)$ implies that $\lambda y_{1}(\cdot)+(1-\lambda) y_{2}(\cdot)$ is a minimizer. From the strict convexity of $\mathcal{F}[\cdot]$ we have then that

$$
\frac{d y_{1}}{d s}-b\left(y_{1}(s), s\right)=\frac{d y_{2}}{d s}-b\left(y_{2}(s), s\right), \quad t \leq s \leq T
$$

Since $y_{1}(t)=y_{2}(t)=y$, we conclude from this last identity that $y_{1}(s)=$ $y_{2}(s), t \leq s \leq T$, and so obtain the uniqueness of the minimizer.

To show that the function $q(x, y, t)$ is $C^{1}$ we consider the optimal control $\lambda^{*}(x, y, t)=y^{\prime}(t)$ where $y(\cdot) \in E_{x, y, t}$ is the unique minimizer for the variational problem (2.2). Evidently $\lambda^{*}(x, y, t)=b(y, t)$ if $y \geq F(x, t)$. We first prove that $\lambda^{*}(x, y, t)$ is continuous in $(x, y, t)$ for $(x, y) \in \mathbb{R}^{2}, t<T$. To do this let $D_{x, y}(\delta) \subset \mathbb{R}^{2}$ be the disc of radius $\delta>0$ centered at $(x, y)$. Then there exists a constant $K(\delta)>0$ depending only on $\delta$ such that

$$
\int_{t}^{T}\left[\frac{d z(s)}{d s}\right]^{2} \leq K(\delta), \quad z(\cdot) \in E_{x^{\prime}, y^{\prime}, t}, \quad\left(x^{\prime}, y^{\prime}\right) \in D_{x, y}(\delta),
$$

where $z(\cdot)$ is the minimizer of the variational problem. To see $(2.20)$, observe that

$$
\mathcal{F}[z(\cdot)] \geq \frac{1}{4} \int_{t}^{T}\left[\frac{d z(s)}{d s}\right]^{2} d s-\frac{1}{2} \int_{t}^{T} b(z(s), s)^{2} d s .
$$

Now from (1.1) one has that

$$
|b(z(s), s)| \leq|b(z(t), s)|+A \int_{t}^{T}\left|\frac{d z}{d s^{\prime}}\right| d s^{\prime}, \quad t \leq s \leq T .
$$

Hence from the Schwarz inequality we have that

$$
\mathcal{F}[z(\cdot)] \geq \frac{1}{8} \int_{t}^{T}\left[\frac{d z(s)}{d s}\right]^{2} d s-K^{\prime}(\delta),
$$

where $K^{\prime}(\delta)$ is a constant depending on $\delta$. Now (2.20) follows from this last inequality and the continuity of the function $q(\cdot, \cdot, t)$ on $D_{x, y}(\delta)$. 
Next we show that for any $\varepsilon>0$ there exists $\delta>0$ such that

$$
\int_{t}^{T}\left[\frac{d y}{d s}-\frac{d z}{d s}\right]^{2} d s<\varepsilon, \quad z(\cdot) \in E_{x^{\prime}, y^{\prime}, t}, \quad\left(x^{\prime}, y^{\prime}\right) \in D_{x, y}(\delta),
$$

where $y(\cdot) \in E_{x, y, t}$ is the minimizer for $(2.2)$ and $z(\cdot) \in E_{x^{\prime}, y^{\prime}, t}$ is also the minimizer. The inequality (2.21) follows from the convexity $(2.19)$ of the functional $\mathcal{F}[\cdot]$. We first consider the situation $y \geq F(x, t)$, where the minimizer $y(\cdot) \in E_{x, y, t}$ satisfies $y^{\prime}(s)=b(y(s), s)$ and $q(x, y, t)=0$. Thus for $\varepsilon_{1}>0$ there exists $\delta_{1}>0$ such that

$$
\mathcal{F}[z(\cdot)]<\varepsilon_{1}, \quad z(\cdot) \in E_{x^{\prime}, y^{\prime}, t}, \quad\left(x^{\prime}, y^{\prime}\right) \in D_{x, y}\left(\delta_{1}\right) .
$$

We can restate $(2.22)$ as $z(\cdot)$ satisfies the initial value problem

$$
\frac{d z}{d s}=b(z(s), s)+f(s), \quad t \leq s \leq T, \quad z(t)=y^{\prime},
$$

where $\|f\|_{2}<\sqrt{2 \varepsilon_{1}}$. Putting now $\varphi(s)=z(s)-y(s)$ it follows from (1.1) that $\varphi(s)$ satisfies the initial value problem

$$
\frac{d \varphi}{d s}=a(s) \varphi(s)+f(s), \quad t \leq s \leq T, \quad \varphi(t)=y^{\prime}-y,
$$

where $\sup _{t \leq s \leq T}|a(s)| \leq A$. It follows that there are positive constants $C_{1}$ and $C_{2}$ such that

$$
\sup _{t \leq s \leq T}|z(s)-y(s)| \leq C_{1}\left|y^{\prime}-y\right|+C_{2} \sqrt{\varepsilon_{1}} .
$$

We write the left-hand side of $(2.21)$ as

$$
\begin{aligned}
& \int_{t}^{T}\{[b(y(s), s)-\left.b(z(s), s)]+\left[b(z(s), s)-\frac{d z}{d s}\right]\right\}^{2} d s \\
& \leq 2 \int_{t}^{T}[b(y(s), s)-b(z(s), s)]^{2}+4 \mathcal{F}[z(\cdot)] .
\end{aligned}
$$

The inequality (2.21) follows from this last inequality, (2.22) and (2.24).

We prove (2.21) for $y<F(x, t)$. First let $\delta_{1}>0$ be such that closure of $D_{x, y}\left(\delta_{1}\right)$ lies in the set $\left\{\left(x^{\prime}, y^{\prime}\right) \in \mathbb{R}^{2}: y^{\prime}<F\left(x^{\prime}, t\right)\right\}$. Then it follows from (2.20) that there exists $\lambda_{0}, 0<\lambda_{0}<1$, such that

$$
\lambda_{0} z(\cdot)+\left(1-\lambda_{0}\right) y(\cdot) \in E, \quad z(\cdot) \in E_{x^{\prime}, y^{\prime}, t}, \quad\left(x^{\prime}, y^{\prime}\right) \in D_{x, y}\left(\delta_{1}\right),
$$

where $y(\cdot) \in E_{x, y, t}$ and $z(\cdot) \in E_{x^{\prime}, y^{\prime}, t}$ are the minimizers for (2.2). Since $z(\cdot)$ and $y(\cdot)$ are also in $E$ we may use the convexity $(2.19)$ of the functional $\mathcal{F}[\cdot]$. In particular we have that

$$
\begin{aligned}
\mathcal{F}\left[\lambda_{0} z(\cdot)+\left(1-\lambda_{0}\right)\right. & y(\cdot)] \leq \lambda_{0} \mathcal{F}[z(\cdot)]+\left(1-\lambda_{0}\right) \mathcal{F}[y(\cdot)] \\
& -\frac{\lambda_{0}\left(1-\lambda_{0}\right)}{2} \int_{t}^{T}\left\{\frac{d y}{d s}-\frac{d z}{d s}+b(z(s), s)-b(y(s), s)\right\}^{2} d s .
\end{aligned}
$$


Using the continuity of the function $q(\cdot, \cdot, t)$ at $(x, y)$ we conclude from the last inequality that there exists $\delta_{2}, 0<\delta_{2}<\delta_{1}$, such that

$$
\begin{aligned}
\frac{1}{2} \int_{t}^{T}\left\{\frac{d y}{d s}-\frac{d z}{d s}+b(z(s), s)-b(y(s), s)\right\}^{2} d s<\varepsilon_{2} \\
z(\cdot) \in E_{x^{\prime}, y^{\prime}, t}, \quad\left(x^{\prime}, y^{\prime}\right) \in D_{x, y}\left(\delta_{2}\right),
\end{aligned}
$$

where again $y(\cdot) \in E_{x, y, t}$ and $z(\cdot) \in E_{x^{\prime}, y^{\prime}, t}$ are the minimizers for (2.2). Here $\varepsilon_{2}>0$ can be chosen arbitrarily and $\delta_{2}$ depends on $\varepsilon_{2}$. Now we may argue as for the case when $y \geq F(x, t)$. Thus letting $\varphi(s)=z(s)-y(s)$ we have that $\varphi(s)$ satisfies the equation (2.23) with $\|f\|_{2}<\sqrt{2 \varepsilon_{2}}$. Hence we obtain an inequality analogous to (2.24), which together with (2.25) implies (2.21).

The continuity of $\lambda^{*}(x, y, t)$ in $(x, y)$ follows easily from (2.21) upon using (2.14). Thus for a minimizer of $(2.2), z(\cdot) \in E_{x^{\prime}, y^{\prime}, t}$ one has

$$
\frac{d z}{d s}-b(z(s), s)=A\left(x^{\prime}, y^{\prime}, t\right) \exp \left[-\int_{t}^{s} \frac{\partial b}{\partial y}\left(z\left(s^{\prime}\right), s^{\prime}\right) d s^{\prime}\right], \quad t \leq s \leq T,
$$

where $\lambda^{*}\left(x^{\prime}, y^{\prime}, t\right)=b\left(y^{\prime}, t\right)+A\left(x^{\prime}, y^{\prime}, t\right)$. Evidently (2.21) implies that the function $A(\cdot, \cdot, t)$ is continuous at $(x, y)$. Finally we observe that the continuity of $\lambda^{*}(x, y, t)$ as a function of $(x, y, t)$ for $(x, y) \in \mathbb{R}^{2}$ and $t<T$ follows from (2.26). In fact if $y(\cdot) \in E_{x, y, t}$ is the minimizer for (2.2) then (2.26) implies that for fixed $x$ the function $s \rightarrow \lambda^{*}(x, y(s), s)$ is continuous for $t \leq s<T$. Hence if we combine this with the previous argument on the continuity of $\lambda^{*}(\cdot, \cdot, t)$ for fixed $t$ we obtain the continuity of $\lambda^{*}(\cdot, \cdot, \cdot)$ in all three variables.

We prove the $C^{1}$ property of the function $q(x, y, t)$ for $(x, y) \in \mathbb{R}^{2}$ and $t<T$. First we observe that the function $q$ is differentiable in at least one direction. Thus

$$
-\left.\frac{d}{d s} q(x, y(s), s)\right|_{s=t}=\frac{1}{2}\left[\lambda^{*}(x, y, t)-b(y, t)\right]^{2},
$$

where $y(\cdot) \in E_{x, y, t}$ is the minimizer for (2.2). We use the continuity of the function $\lambda^{*}(\cdot, \cdot, \cdot)$ to show differentiability in the other directions. Let us assume that $y<$ $F(x, t)$ and $\Delta y$ are small enough so that $|\Delta y|<F(x, t)-y$. Then

$$
\begin{aligned}
& q(x, y+\Delta y, t)-q(x, y, t) \leq-\frac{1}{2} \int_{t}^{T}\left[\lambda^{*}(s)-b(y(s), s)\right]^{2} d s \\
& \quad+\frac{1}{2} \int_{t}^{T}\left[\lambda^{*}(s)-\Delta y /(T-t)-b(y(s)+(T-s) \Delta y /(T-t), s)\right]^{2} d s,
\end{aligned}
$$

where $y(\cdot) \in E_{x, y, t}$ is the minimizer for $(2.2)$ and $\lambda^{*}(s)=y^{\prime}(s)$ for $t \leq s \leq T$. Letting $\Delta y \rightarrow 0$ in (2.28) we conclude that

$$
\begin{aligned}
\limsup _{\Delta y \rightarrow 0} & \frac{q(x, y+\Delta y, t)-q(x, y, t)}{\Delta y} \\
& \leq-\frac{1}{T-t} \int_{t}^{T}\left[1+(T-s) \frac{\partial b}{\partial y}(y(s), s)\right]\left[\lambda^{*}(s)-b(y(s), s)\right] d s .
\end{aligned}
$$


Alternatively let $y_{\Delta}(\cdot) \in E_{x, y+\Delta y, t}$ be the minimizer for (2.2) and suppose $\lambda_{\Delta}^{*}(s)=y_{\Delta}^{\prime}(s)$ for $t \leq s \leq T$. Then one also has

$$
\begin{aligned}
& q(x, y+\Delta y, t)-q(x, y, t) \geq \frac{1}{2} \int_{t}^{T}\left[\lambda_{\Delta}^{*}(s)-b\left(y_{\Delta}(s), s\right)\right]^{2} d s \\
& \quad-\frac{1}{2} \int_{t}^{T}\left[\lambda_{\Delta}^{*}(s)+\Delta y /(T-t)-b\left(y_{\Delta}(s)-(T-s) \Delta y /(T-t), s\right)\right]^{2} d s .
\end{aligned}
$$

It follows from (2.30) by using (2.21), (2.26), and the continuity of the function $\lambda^{*}(\cdot, \cdot, \cdot)$ that

$$
\begin{aligned}
\liminf _{\Delta y \rightarrow 0} & \frac{q(x, y+\Delta y, t)-q(x, y, t)}{\Delta y} \\
& \geq-\frac{1}{T-t} \int_{t}^{T}\left[1+(T-s) \frac{\partial b}{\partial y}(y(s), s)\right]\left[\lambda^{*}(s)-b(y(s), s)\right] d s .
\end{aligned}
$$

The differentiability of $q(x, y, t)$ with respect to $y$ follows from (2.29) and (2.31). Using (2.21), (2.26) again we also see from the formula on the right-hand side of $(2.29)$ that $\partial q(x, y, t) / \partial y$ is continuous in $(x, y, t)$ for $y<F(x, t)$ and $t<T$. It is easy to extend this argument to show that $\partial q(x, y, t) / \partial y$ exists for all $y \in \mathbb{R}$ and the derivative is continuous in $(x, y, t)$ for $(x, y) \in \mathbb{R}^{2}$ and $t<T$. This follows from the fact that the formula on the right-hand side of $(2.29)$ is zero if $y=F(x, t)$.

One can see by a similar argument that $q(x, y, t)$ is differentiable with respect to $x$ and that $\partial q(x, y, t) / \partial x$ is continuous for $(x, y) \in \mathbb{R}^{2}$ and $t<T$. Finally (2.27) and the fact that $\partial q(x, y, t) / \partial y$ is continuous shows that $q(x, y, t)$ is differentiable with respect to $t$ and $\partial q(x, y, t) / \partial t$ is continuous in $(x, y, t)$ for $(x, y) \in \mathbb{R}^{2}$ and $t<T$. We have shown that the function $q(x, y, t)$ is $C^{1}$ for $(x, y) \in \mathbb{R}^{2}$ and $t<T$.

Corollary 2.3. Assume $b(\cdot, \cdot)$ satisfies the conditions of Proposition $2.2, q(x, y, t)$ is the function defined by $(2.2)$, and $\lambda^{*}(x, y, t)$ is the corresponding optimal control for $(x, y) \in \mathbb{R}^{2}$ and $t<T$. Then there are the identities

$$
\begin{aligned}
\partial q(x, y, t) / \partial y & =b(y, t)-\lambda^{*}(x, y, t), \\
\partial q(x, y, t) / \partial t & =\frac{1}{2}\left[\lambda^{*}(x, y, t)^{2}-b(y, t)^{2}\right] .
\end{aligned}
$$

Furthermore, for $y<F(x, t)$ there are the inequalities

$$
\frac{\partial q(x, y, t)}{\partial y}<0, \quad \frac{\partial q(x, y, t)}{\partial x}>0 .
$$

Proof. We first show the identity (2.32) for $\partial q(x, y, t) / \partial y$. We assume $y<F(x, t)$ since it is obvious otherwise. Using the fact that $q(x, y, t)$ is the minimizer for the variational problem (2.2) we have that, for $\lambda \in \mathbb{R}$,

$$
q(x, y, t) \leq \frac{1}{2}[\lambda-b(y, t)]^{2} \Delta t+q(x, y+\lambda \Delta t, t+\Delta t)+O\left[(\Delta t)^{2}\right]
$$


Since $q$ is $C^{1}$ this implies that

$$
\frac{1}{2}\left[\lambda^{*}(x, y, t)^{2}-b(y, t)\right]^{2} \leq \frac{1}{2}[\lambda-b(y, t)]^{2}+\left[\lambda-\lambda^{*}(x, y, t)\right] \frac{\partial q}{\partial y}(x, y, t),
$$

$\lambda \in \mathbb{R}$, where we have used (2.27). The inequality (2.34) implies the first identity of (2.32). The second identity follows from the first identity and (2.27).

The first inequality of (2.33) follows from Proposition 2.1. To show that $\partial q(x, y, t) / \partial x>0$ we derive a formula for $\partial q(x, y, t) / \partial x$ similar to the formula (2.32) for $\partial q(x, y, t) / \partial y$. We have already seen that $\partial q(x, y, t) / \partial x$ is given by the expression

$$
\frac{\partial q}{\partial x}(x, y, t)=\frac{1}{T-t} \int_{t}^{T}\left[1-(s-t) \frac{\partial b}{\partial y}(y(s), s)\right]\left[\lambda^{*}(s)-b(y(s), s)\right] d s
$$

similar to the right-hand side of (2.29). Adding (2.29) and (2.35) we conclude that

$$
\frac{\partial q}{\partial y}(x, y, t)+\frac{\partial q}{\partial x}(x, y, t)=-\int_{t}^{T} \frac{\partial b}{\partial y}(y(s), s)\left[\lambda^{*}(s)-b(y(s), s)\right] d s .
$$

If we use now the identity (2.26) we conclude from the previous expression that

$$
\frac{\partial q}{\partial x}(x, y, t)=\left[\lambda^{*}(x, y, t)-b(y, t)\right] \exp \left[-\int_{t}^{T} \frac{\partial b}{\partial y}(y(s), s) d s\right],
$$

where $y(\cdot) \in E_{x, y, t}$ is the minimizer for (2.2). Proposition 2.1 and (2.37) now imply $\partial q(x, y, t) / \partial x>0$.

Remark 2.4. Observe that Proposition 2.2 and Corollary 2.3 imply that $q(x, y, t)$ is a classical solution to the $\varepsilon=0$ Hamilton-Jacobi equation (1.8).

Next we show that $q(x, y, t)$ is twice differentiable in $(x, y)$. Since this is obvious for $y>F(x, t)$ we consider $y<F(x, t)$. Let $\varphi(s), t \leq s \leq T$, be the solution of the first variation equation (2.16) with terminal data $\varphi(T)=0$ and $\varphi^{\prime}(T)=-1$. Then one should have the identity

$$
\partial \lambda^{*}(x, y, t) / \partial y=\varphi^{\prime}(t) / \varphi(t) .
$$

We have already given an argument to show $\varphi(s)>0$ for $t \leq s \leq T$, if we assume $b(\cdot, s)$ is concave for $t \leq s \leq T$. Hence in this case the right-hand side of (2.38) makes sense. Note also that we may write (2.16) in the form

$$
\left[\frac{d}{d s}+\frac{\partial b}{\partial y}(y(s), s)\right]\left[\frac{d \varphi}{d s}-\frac{\partial b}{\partial y}(y(s), s) \varphi(s)\right]-V(s) \varphi(s)=0,
$$

where $V(s) \geq 0$ if $b(\cdot, s)$ is concave for all $s, t \leq s \leq T$. Hence it follows from (2.39) that if we assume the concavity of $b(\cdot, s) t \leq s \leq T$, then $\varphi^{\prime}(t)-\partial b / \partial y(y(t), t) \varphi(t)$ $<0$. Thus from (2.32) and (2.38) we conclude that $\partial^{2} q(x, y, t) / \partial y^{2}>0$. We make this argument rigorous in the following proposition. 
Proposition 2.5. Assume the function $b(\cdot, \cdot)$ satisfies $(1.1)$ and that $b(y, s)$ is concave in $y$ for $y \in \mathbb{R}$ and $s \leq T$. Then the function $q(x, y, t)$ of $(2.2)$ is convex in $(x, y)$ for $(x, y) \in \mathbb{R}^{2}$ and $t<T$. Suppose in addition that $b(y, s)$ is twice differentiable in $y$ for $y \in \mathbb{R}$ and $s \leq T$, and $\partial^{2} b(y, s) / \partial y^{2}$ is continuous in $(y, s)$. Then $q(x, y, t)$ is twice differentiable in $(x, y)$ for $(x, y, t) \in U_{T}=\left\{(x, y, t):(x, y) \in \mathbb{R}^{2}\right.$, $t<T, y<F(x, t)\}$. The second derivatives of $q(x, y, t)$ with respect to $(x, y)$ are continuous in $U_{T}$ and satisfy $\partial^{2} q(x, y, t) / \partial x^{2}>0, \partial^{2} q(x, y, t) / \partial y^{2}>0$, and $\partial^{2} q(x, y, t) / \partial x \partial y<0$. Furthermore, if $\left(x_{0}, y_{0}, t_{0}\right) \in \partial U_{T}$ and $t_{0}<T$ then

$$
\lim _{(x, y, t) \rightarrow\left(x_{0}, y_{0}, t_{0}\right)} \partial^{2} q(x, y, t) / \partial x^{2}>0, \quad \lim _{(x, y, t) \rightarrow\left(x_{0}, y_{0}, t_{0}\right)} \partial^{2} q(x, y, t) / \partial y^{2}>0 .
$$

Proof. Observe that the function $F(x, t), x \in \mathbb{R}, t<T$, defined by (1.11) is a convex function of $x$. In fact one has

$$
\frac{\partial F}{\partial x}(x, t)=\exp \left[-\int_{t}^{T} \frac{\partial b}{\partial y}(y(s), s) d s\right]
$$

where $y(s), s \leq T$, is the solution to (1.11). Hence, by the concavity of $b(\cdot, s)$ for $t \leq s \leq T$, one has that $\partial F(x, t) / \partial x$ is an increasing function of $x$. It follows that the set $V_{t}=\left\{(x, y) \in \mathbb{R}^{2}: y \geq F(x, t)\right\}$ on which $q(\cdot, \cdot, t)$ vanishes is convex. We also have from the argument of Proposition 2.2 that $q(x, y, t)$ is locally convex on the not necessarily convex open set $\mathbb{R}^{2} \backslash V_{t}$. Hence $q(x, y, t)$ is convex in $(x, y)$ for all $(x, y) \in \mathbb{R}^{2}$.

We assume now $b(y, s)$ is twice continuously differentiable in $y$ for $y \in \mathbb{R}$ and $s \leq T$. We can write $(2.39)$ as a system

$$
\begin{aligned}
& \frac{d \varphi}{d s}-\frac{\partial b}{\partial y}(y(s), s) \varphi(s)=-\psi(s), \quad t \leq s \leq T \\
& \frac{d \psi}{d s}+\frac{\partial b}{\partial y}(y(s), s) \psi(s)=-V(s) \varphi(s), \quad t \leq s \leq T,
\end{aligned}
$$

where $y(\cdot) \in E_{x, y, t}$ is the minimizer for (2.2). Evidently (2.42) has a unique solution $[\varphi(s), \psi(s)]$ defined for $t \leq s \leq T$, with terminal data $\varphi(T)=0$ and $\psi(T)=1$. Multiplying the first equation in $(2.42)$ by $\psi(s)$ and the second by $\varphi(s)$ we see, upon integrating, that

$$
\psi(s) \varphi(s)=\int_{s}^{T} \psi\left(s^{\prime}\right)^{2}+V\left(s^{\prime}\right) \varphi\left(s^{\prime}\right)^{2} d s^{\prime}, \quad t \leq s \leq T .
$$

From the terminal conditions on $[\varphi(s), \psi(s)]$ we have that $\varphi(s)>0$ and $\psi(s)>0$ for $s$ close to $T$. It follows then from $(2.43)$ that $\varphi(s)>0$ and $\psi(s)>0$ for $t \leq s \leq T$.

Next we use (2.26) to write the equation for the minimizer $y(\cdot) \in E_{x, y, t}$ of $(2.2)$ in a form similar to $(2.42)$. Thus we have

$$
\begin{aligned}
& \frac{d y}{d s}-b(y(s), s)=-p(s), \quad t \leq s \leq T \\
& \frac{d p}{d s}+\frac{\partial b}{\partial y}(y(s), s) p(s)=0, \quad t \leq s \leq T
\end{aligned}
$$


In (2.44) the first equation is the definition of the Hamiltonian momentum $p(s)$ while the second equation is equivalent to (2.26). Suppose now $z(\cdot) \in E_{x^{\prime}, y^{\prime}, t}$ is also a minimizer for $(2.2)$ and define $\Phi(s)=z(s)-y(s)$ and $\Psi(s)=P(s)-p(s)$, where $P(s)$ is the momentum corresponding to $z(\cdot)$. Then since $z(\cdot)$ satisfies an equation similar to (2.44) we have that

$$
\begin{aligned}
& \frac{d \Phi}{d s}-\Phi(s) \int_{0}^{1} \frac{\partial b}{\partial y}(\mu y(s)+(1-\mu) z(s), s) d \mu=-\Psi(s), t \leq s \leq T \\
& \frac{d \Psi}{d s}+\frac{\partial b}{\partial y}(z(s), s) \Psi(s)=-\Phi(s) p(s) \int_{0}^{1} \frac{\partial^{2} b}{\partial y^{2}}(\mu y(s)+(1-\mu) z(s), s) d \mu, t \leq s \leq T
\end{aligned}
$$

We consider now the situation where $x^{\prime}=x$ so $\Phi(T)=0$. Then if $y^{\prime}=y+\Delta y$ we may write

$$
\Phi(t)=\alpha(\Delta y) \Psi(T), \quad \Psi(t)=\beta(\Delta y) \Psi(T),
$$

where the functions $\alpha(\cdot)$ and $\beta(\cdot)$ satisfy

$$
\lim _{\Delta y \rightarrow 0} \alpha(\Delta y)=\varphi(t), \quad \lim _{\Delta y \rightarrow 0} \beta(\Delta y)=\psi(t)
$$

since the coefficients in the equations (2.45) converge as $\Delta y \rightarrow 0$ to the coefficients in the equations $(2.42)$. Now we have that

$$
\frac{\lambda^{*}(x, y+\Delta y, t)-\lambda^{*}(x, y, t)}{\Delta y}=\frac{1}{\Delta y}\left[\Phi(t) \int_{0}^{1} \frac{\partial b}{\partial y}(\mu y(s)+(1-\mu) z(s), s) d \mu-\Psi(t)\right]
$$

and $\Phi(t)=\Delta y$. Hence it follows from (2.46), (2.47) that $\lambda^{*}(x, y, t)$ is differentiable with respect to $y$ and

$$
\partial \lambda^{*}(x, y, t) / \partial y=\partial b(y, t) / \partial y-\psi(t) / \varphi(t) .
$$

One also sees easily from the representation (2.48) that $\partial \lambda^{*}(x, y, t) / \partial y$ is continuous in $U_{T}$ and that the limit exists as $(x, y, t) \rightarrow\left(x_{0}, y_{0}, t_{0}\right) \in \partial U_{T}$ provided $t_{0}<T$. The fact that $\partial^{2} q(x, y, t) / \partial y^{2}>0$ follows now from (2.32) and the fact that $\psi(t)>0$ and $\varphi(t)>0$.

We can similarly see that $\lambda^{*}(x, y, t)$ is differentiable with respect to $x$, that $\partial \lambda^{*}(x, y, t) / \partial x$ is continuous in $U_{T}$, and that the limit exists as $(x, y, t) \rightarrow\left(x_{0}, y_{0}, t_{0}\right)$ $\in \partial U_{T}$ provided $t_{0}<T$. To see that $\partial^{2} q(x, y, t) / \partial x \partial y<0$ we note that

$$
\partial^{2} q(x, y, t) / \partial x \partial y=\psi(t) / \varphi(T),
$$

where $[\varphi(s), \psi(s)]$, defined for $t \leq s \leq T$, is the solution of (2.42) with initial data $\varphi(t)=0$ and $\psi(t)=1$. We have in this case

$$
\psi(s) \varphi(s)=-\int_{t}^{s} \psi\left(s^{\prime}\right)^{2}+V\left(s^{\prime}\right) \varphi\left(s^{\prime}\right)^{2} d s^{\prime},
$$

whence $\varphi(T)<0$ and so $\partial^{2} q(x, y, t) / \partial x \partial y$ is negative. 
To prove the twice differentiability of $q(x, y, t)$ with respect to $x$ we use the representation

$$
\partial q(x, y, t) / \partial x=p(T)
$$

where $p(s)$ is given by (2.44) for the minimizer $y(\cdot) \in E_{x, y, t}$ of (2.2). The differentiability of $\partial q(x, y, t) / \partial x$ and the positivity of $\partial^{2} q(x, y, t) / \partial x^{2}$ proceeds as before by representing $\partial^{2} q(x, y, t) / \partial x^{2}$ in terms of a solution to (2.42). Finally we observe that (2.49) follows from (2.26), (2.32), and (2.36).

Remark 2.6. Proposition 2.5 shows that all second derivatives of $q(x, y, t)$ with respect to $(x, y)$ have jump discontinuities across the boundary $y=F(x, t)$. Hence $q(x, y, t)$ is not $C^{2}$ in $(x, y)$ for all $(x, y) \in \mathbb{R}^{2}$.

\section{Proof of Theorem 1.1}

Our main goal in this section is to show that the function $q_{\varepsilon}(x, y, t)$ defined by $(1.7)$ converges as $\varepsilon \rightarrow 0$ to the function $q(x, y, t)$ defined by (1.10). The formula (1.15) for $q_{\varepsilon}(x, y, t)$ makes this intuitively clear, but it is not obvious under what circumstances the function defined by (1.7) has the representation (1.15). As part of our proof of convergence we shall make use of various situations in which (1.15) is valid. First we regularize the terminal data (1.9).

Lemma 3.1. Suppose $b(\cdot, \cdot)$ satisfies (1.1) and $q_{\varepsilon}(x, y, t)$ is given by (1.7). Then there exists $\delta>0$ and universal positive constants $C_{1}$ and $C_{2}$ such that, if $T-t<\delta$ and $\varepsilon<1$, the inequality

$$
C_{1}(x-y)^{2} /(T-t)<q_{\varepsilon}(x, y, t)<C_{2}(x-y)^{2} /(T-t),
$$

holds for $y$ in the region

$$
x-y>2 \int_{t}^{T}|b(x, s)| d s+\sqrt{\varepsilon(T-t)} .
$$

Proof. Since $b(\cdot, \cdot)$ satisfies (1.1) one can uniquely solve the stochastic equation (1.5) with given initial data. The solution $u_{\varepsilon}(x, y, t)$ of the terminal value problem given by (1.2) and (1.3) is then the probability (1.6). Letting $Z_{\varepsilon}(s)=Y_{\varepsilon}(s)-y$, we have then that

$$
\begin{aligned}
Z_{\varepsilon}(s)= & \int_{t}^{s}\left[\int_{0}^{1} d \mu \frac{\partial b}{\partial y}\left(\mu Y_{\varepsilon}\left(s^{\prime}\right)+(1-\mu) y, s^{\prime}\right)\right] Z_{\varepsilon}\left(s^{\prime}\right) d s^{\prime} \\
& +\int_{t}^{s} b\left(y, s^{\prime}\right) d s^{\prime}+\sqrt{\varepsilon}[W(s)-W(t)], \quad s>t .
\end{aligned}
$$

Now applying Gronwall's inequality to (3.3) we conclude that

$$
\sup _{t \leq s \leq T}\left|Z_{\varepsilon}(s)\right| \leq A(t, T) \sup _{t \leq s \leq T}\left|\int_{t}^{s} b\left(y, s^{\prime}\right) d s^{\prime}+\sqrt{\varepsilon}[W(s)-W(t)]\right|,
$$

where $A(t, T)$ is a constant depending only on $t$ and $T$. The lower bound in inequality (3.1) follows from (3.4), (1.6) and (1.7). 
To obtain the upper bound we consider the stochastic process $Z_{\varepsilon}^{\prime}(s), s \geq t$, defined by the equation

$$
d Z_{\varepsilon}^{\prime}(s)=\left[A Z_{\varepsilon}^{\prime}(s)-b(x, s)\right] d s+\sqrt{\varepsilon} d W(s), \quad Z_{\varepsilon}^{\prime}(t)=x-y,
$$

where $A$ is the constant in (1.1). If $\tau$ is the first hitting time at $x$ for the process $Y_{\varepsilon}(s)$ of $(1.5)$ with $Y_{\varepsilon}(t)=y$, then it is evident that $Z_{\varepsilon}^{\prime}(s) \geq x-Y_{\varepsilon}(s)$ for $t \leq s \leq \tau$. It follows that

$$
P(\tau<T) \geq P\left(Z_{\varepsilon}^{\prime}(T)<0 \mid Z_{\varepsilon}^{\prime}(t)=x-y\right) .
$$

Since the stochastic equation (3.5) is exactly solvable, we can estimate the right-hand side of (3.6). Assuming $x-y$ satisfies (3.2) we conclude that

$$
P\left(Z_{\varepsilon}^{\prime}(T)<0 \mid Z_{\varepsilon}^{\prime}(t)=x-y\right) \geq \exp \left[-C(x-y)^{2} / \varepsilon(T-t)\right],
$$

for a constant $C$ depending only on the parameter $A$ in (3.5). The upper bound in (3.1) follows now from (3.6), (3.7), and the inequality

$$
P\left(Y_{\varepsilon}(T)>x \mid Y_{\varepsilon}(t)=y\right) \geq P(\tau<T) \inf _{t \leq s \leq T} P\left(Y_{\varepsilon}(T)>x \mid Y_{\varepsilon}(s)=x\right),
$$

since it is clear that for $\delta$ small enough the infimum in (3.8) is larger than $1 / 4$.

We consider a controller $\lambda_{\varepsilon}(y, s)$ for $y \in \mathbb{R}$ and $s<T$, which is uniformly Lipschitz in $y$ for $t \leq s \leq T-\delta$. Thus there is a constant $C$ such that

$$
\left|\lambda_{\varepsilon}(y, s)-\lambda_{\varepsilon}\left(y^{\prime}, s\right)\right| \leq C\left|y-y^{\prime}\right|, \quad y, y^{\prime} \in \mathbb{R}, \quad t \leq s \leq T-\delta .
$$

Hence we may solve the stochastic differential equation (1.14) for $t \leq s \leq T-\delta$. We show that in this case the the expectation on the right-hand side of (1.15) is bounded below by the left-hand side.

Lemma 3.2. Suppose $\lambda_{\varepsilon}(\cdot, \cdot)$ satisfies (3.9) and $b(\cdot, \cdot)$ satisfies (1.1). Then if $q_{\varepsilon}(x, y, t)$ is given by $(1.7)$ the inequality

$$
\begin{array}{r}
q_{\varepsilon}(x, y, t) \leq E\left\{\frac{1}{2} \int_{t}^{T-\delta}\left[\lambda_{\varepsilon}\left(y_{\varepsilon}(s), s\right)-b\left(y_{\varepsilon}(s), s\right)\right]^{2} d s\right. \\
\left.+q_{\varepsilon}\left(x, y_{\varepsilon}(T-\delta), T-\delta\right) \mid y_{\varepsilon}(t)=y\right\}
\end{array}
$$

holds, where $y_{\varepsilon}(\cdot)$ is the solution to the SDE (1.14).

Proof. Let $V_{\varepsilon}(y, s)$ for $y \in \mathbb{R}$ and $s \leq T-\delta$, denote the right-hand side of (3.10). Arguing as in the proof of Lemma 3.1, one sees that

$$
0<V_{\varepsilon}(y, s) \leq A y^{2}+B, \quad y \in \mathbb{R}, \quad t \leq s \leq T-\delta,
$$

for some constants $A$ and $B$. In addition $V_{\varepsilon}, \partial V_{\varepsilon} / \partial s, \partial V_{\varepsilon} / \partial y$, and $\partial^{2} V_{\varepsilon} / \partial y^{2}$ are all continuous functions of $(y, s)$ for $y \in \mathbb{R}$ and $t \leq s<T-\delta$, and satisfy the equation

$$
\frac{\partial V_{\varepsilon}}{\partial s}+\lambda_{\varepsilon}(y, s) \frac{\partial V_{\varepsilon}}{\partial y}+\frac{\varepsilon}{2} \frac{\partial^{2} V_{\varepsilon}}{\partial y^{2}}+\frac{1}{2}\left[\lambda_{\varepsilon}(y, s)-b(y, s)\right]^{2}=0,
$$


for $y \in \mathbb{R}, t \leq s<T-\delta$, with terminal condition

$$
V_{\varepsilon}(y, T-\delta)=q_{\varepsilon}(x, y, T-\delta), \quad y \in \mathbb{R} .
$$

Note that the twice differentiability of $V_{\varepsilon}(y, s)$ with respect to $y$ uses the fact that the function $\lambda_{\varepsilon}(\cdot, s)-b(\cdot, s)$ is Lipschitz continuous for $t \leq s \leq T-\delta$ (see Theorem 9 in Chapter 1 of [7]). From (1.8) and (3.12) we conclude that the function $W_{\varepsilon}(y, s)=V_{\varepsilon}(y, s)-q_{\varepsilon}(x, y, s)$ satisfies the PDE

$$
\frac{\partial W_{\varepsilon}}{\partial s}+\lambda_{\varepsilon}(y, s) \frac{\partial W_{\varepsilon}}{\partial y}+\frac{\varepsilon}{2} \frac{\partial^{2} W_{\varepsilon}}{\partial y^{2}}+\frac{1}{2}\left[\lambda_{\varepsilon}(y, s)-b(y, s)+\frac{\partial q_{\varepsilon}}{\partial y}\right]^{2}=0,
$$

for $y \in R, t \leq s \leq T-\delta$, and all the derivatives $\partial W_{\varepsilon} / \partial s, \partial W_{\varepsilon} / \partial y$, and $\partial^{2} W_{\varepsilon} / \partial y^{2}$ are continuous. Furthermore by (3.13) the terminal condition for $W_{\varepsilon}$ is $W_{\varepsilon}(y, T-$ $\delta)=0, y \in \mathbb{R}$. It follows then from Lemma 3.1, (3.11), and the maximum principle (see Theorem 9 in Chapter 2 of [7]) that $W_{\varepsilon}(y, t) \geq 0, y \in \mathbb{R}$, whence the result follows.

Lemma 3.3. Suppose $b(\cdot, \cdot)$ satisfies (1.1). Then for $x, y \in \mathbb{R}, t<T$, and $\varepsilon<1$, the inequality

$$
q_{\varepsilon}(x, y, t) \leq q(x, y, t)+C(x, y, t, T) \sqrt{\varepsilon}
$$

holds, where $q(x, y, t)$ is given by (1.10) and $C(x, y, t, T)$ is a constant independent of $\varepsilon$.

Proof. Let $y(s)$, defined for $t \leq s \leq T$, be a minimizer for (1.10), whose existence has been established by Proposition 2.1. We set $\lambda_{\varepsilon}(y, s)=\lambda(s)=y^{\prime}(s)$ for $y \in \mathbb{R}$ and $t \leq s \leq T$, and apply Lemma 3.2, taking $\delta=\sqrt{\varepsilon}$. We consider first the case $y \leq F(x, t)$ so $y(T)=x$. Hence $x-y(T-\delta)<C \sqrt{\varepsilon}$ for some constant $C$. It follows then from Lemma 3.1 that

$$
E\left\{q_{\varepsilon}\left(x, y_{\varepsilon}(T-\delta), T-\delta\right) \mid y_{\varepsilon}(t)=y\right\} \leq C_{1} \sqrt{\varepsilon}
$$

for some constant $C_{1}$. Here we are using the fact that $y_{\varepsilon}(s)-y(s)=\sqrt{\varepsilon}[W(s)-$ $W(t)]$ and that $q_{\varepsilon}(x, y, T-\delta)$ is a decreasing positive function of $y \in \mathbb{R}$. We can similarly see that

$$
E\left\{\frac{1}{2} \int_{t}^{T-\delta}\left[\lambda(s)-b\left(y_{\varepsilon}(s), s\right)\right]^{2} d s \mid y_{\varepsilon}(t)=y\right\} \leq q(x, y, t)+C_{2} \sqrt{\varepsilon} .
$$

for some constant $C_{2}$. Thus (3.14) follows from (3.15) and (3.16) in the case $y \leq$ $F(x, t)$. For $y>F(x, t)$ we may use the same argument, noting that $q_{\varepsilon}(x, \cdot, T-\delta)$ is a decreasing positive function.

To obtain a lower bound for $q_{\varepsilon}(x, y, t)$ corresponding to the upper bound established in Lemma 3.3 we shall need to use the fact that the function $\partial q_{\varepsilon}(x, y, s) / \partial y$ is uniformly Lipschitz continuous in $y$ for $(y, s)$ in any region $\left\{(y, s): y \geq y_{0}, t \leq\right.$ $s \leq T-\delta\}$, where $\delta>0, y_{0} \in \mathbb{R}$ can be chosen arbitrarily. 
Lemma 3.4. Suppose $b(\cdot, \cdot)$ satisfies (1.1) and $u_{\varepsilon}(x, y, t)$ for $t<T$ and $y \in \mathbb{R}$, is the unique bounded solution to (1.2) and (1.3). Then for any $\delta>0, y_{0} \in \mathbb{R}$, and $t<T$, there is a positive constant $C\left(\delta, y_{0}, t\right)$ such that

$$
\begin{aligned}
& u_{\varepsilon}(x, y, s) \geq 1 / C\left(\delta, y_{0}, t\right), \quad y \geq y_{0}, t \leq s \leq T-\delta, \\
& \left|\partial u_{\varepsilon}(x, y, s) / \partial y\right|+\left|\partial^{2} u_{\varepsilon}(x, y, s) / \partial y^{2}\right| \leq C\left(\delta, y_{0}, t\right), y \geq y_{0}, t \leq s \leq T-\delta .
\end{aligned}
$$

Proof. To prove the first inequality in (3.17) we proceed as in the proof of Lemma 3.1, using the representation (1.6). Since the solution $Y_{\varepsilon}(s)$ of $(1.5)$ which has initial condition $Y_{\varepsilon}(t)=y$ satisfies the inequality (3.4), it follows that there exists $y_{1}>x$ with the property that $u_{\varepsilon}\left(x, y_{1}, s\right) \geq 1 / 2$ for $t \leq s \leq T$. We consider now $y$ in the interval $y_{0}<y<y_{1}$. Let $\alpha$ be defined by

$$
\alpha=\inf \left\{b\left(y^{\prime}, s\right): y_{0}-1 \leq y^{\prime} \leq y_{1}, t \leq s \leq T\right\},
$$

and $Z_{\varepsilon}(s)$ satisfy the stochastic equation

$$
d Z_{\varepsilon}(s)=\alpha d s+\sqrt{\varepsilon} d W(s), \quad Z_{\varepsilon}(t)=y .
$$

Then $Y_{\varepsilon}(s) \geq Z_{\varepsilon}(s)$ for $t \leq s \leq \tau$, where $\tau$ is the first exit time of $Z_{\varepsilon}(s)$ from the interval $\left[y_{0}-1, y_{1}\right]$. We can easily estimate from below $P\left(\tau<T, Z_{\varepsilon}(\tau)=y_{1}\right)$. Combining this with (3.4) we see that the first inequality in (3.17) holds for $y_{0}<y<y_{1}$.

We turn to the problem of estimating the derivatives in (3.17). Let $y_{1} \in \mathbb{R}$ and $T_{1} \leq T$. We shall be interested in constructing the solution to the terminalboundary value problem

$$
\begin{cases}\frac{\partial w}{\partial t}+b(y, t) \frac{\partial w}{\partial y}+\frac{\varepsilon}{2} \frac{\partial^{2} w}{\partial y^{2}}=0, & y_{1}-\eta<y<y_{1}+\eta, \quad t<T_{1} \\ w\left(y, T_{1}\right)=w_{0}(y), & y \in\left[y_{1}-\eta, y_{1}+\eta\right], \\ w\left(y_{1}-\eta, s\right)=w_{-}(s), & w\left(y_{1}+\eta, s\right)=w_{+}(s), \quad s \leq T_{1}\end{cases}
$$

where $\eta>0$ and the functions $w_{0}(\cdot), w_{-}(\cdot)$ and $w_{+}(\cdot)$ are assumed to be continuous on their domains. The solution to (3.18) can be represented in terms of the Dirchlet Green's function $G\left(y, y^{\prime}, t, T_{1}\right)$ for the problem. Thus

$$
\begin{aligned}
w(y, t)= & \int_{y_{1}-\eta}^{y_{1}+\eta} G\left(y, y^{\prime}, t, T_{1}\right) w_{0}\left(y^{\prime}\right) d y^{\prime}+\varepsilon \int_{t}^{T_{1}} d s w_{-}(s) \frac{\partial G}{\partial y^{\prime}}\left(y, y_{1}-\eta, t, s\right) \\
& -\varepsilon \int_{t}^{T_{1}} d s w_{+}(s) \frac{\partial G}{\partial y^{\prime}}\left(y, y_{1}+\eta, t, s\right) .
\end{aligned}
$$

We shall show that the Green's function may be constructed by perturbation expansion provided $t<T_{1}$ lies in an interval $t \in\left[T_{1}-\Delta, T_{1}\right]$ where $\Delta$ and $\eta$ satisfy the inequalities

$$
\Delta \leq \frac{\eta^{2}}{\varepsilon}, \quad \Delta \leq \frac{\nu \varepsilon}{\left[\sup \left\{|b(y, s)|: y_{1}-\eta \leq y \leq y_{1}+\eta, T_{1}-\Delta \leq s \leq T_{1}\right\}\right]^{2}}
$$

for some $\nu<1$ independent of $b(\cdot, \cdot)$ and $\varepsilon$. 
We construct the Green's function by the standard method [7]. Thus let $G_{D}\left(y, y^{\prime}, t\right)$ be the Green's function for the heat equation on the interval $[-1,1]$ with Dirichlet boundary conditions. The function $G_{D}$ is given from the method of images as an infinite series:

$$
G_{D}\left(y, y^{\prime}, t\right)=\sum_{m=0}^{\infty}(-1)^{p(m)} G\left(y-y_{m}^{\prime}, t\right),
$$

where $y_{0}^{\prime}=y^{\prime}$ and $y_{m}^{\prime}, m \geq 1$, are the multiple reflections of $y^{\prime}$ in the boundaries $y=-1$ and $y=1$, with $p(m)$ being the parity of the reflection, $p(0)=0$. The function $G(y, t)$ is a Gaussian with mean 0 and variance $t$. We now set $K\left(y, y^{\prime}, t, s\right)$ to be

$$
K\left(y, y^{\prime}, t, s\right)=\eta^{-1} G_{D}\left(\frac{y-y_{1}}{\eta}, \frac{y^{\prime}-y_{1}}{\eta}, \frac{\varepsilon(s-t)}{\eta^{2}}\right),
$$

for $y, y^{\prime} \in\left[y_{1}-\eta, y_{1}+\eta\right], t<s$.

The Green's function $G\left(y, y^{\prime}, t, T_{1}\right)$ is formally given by an expansion in terms of the function $K$. et $\mathcal{L}_{t, y}$ denote the operator on the left-hand side of (3.18), so $(3.18)$ is $\mathcal{L}_{t, y} w=0$. Then

$$
\begin{aligned}
G\left(y, y^{\prime}, t, T_{1}\right) & =K\left(y, y^{\prime}, t, T_{1}\right)-\sum_{n=0}^{\infty} v_{n}\left(y, y^{\prime}, t, T_{1}\right), \\
v_{n}\left(y, y^{\prime}, t, T_{1}\right) & =-\int_{t}^{T_{1}} d s \int_{y_{1}-\eta}^{y_{1}+\eta} d z K(y, z, t, s) g_{n}\left(z, y^{\prime}, s, T_{1}\right), \\
g_{0}\left(y, y^{\prime}, t, T_{1}\right) & =\mathcal{L}_{t, y} K\left(y, y^{\prime}, t, T_{1}\right), \\
g_{n+1}\left(y, y^{\prime}, t, T_{1}\right) & =\int_{t}^{T_{1}} d s \int_{y_{1}-\eta}^{y_{1}+\eta} d z \mathcal{L}_{t, y} K(y, z, t, s) g_{n}\left(z, y^{\prime}, s, T_{1}\right) .
\end{aligned}
$$

One easily obtains from (3.23) the estimate

$$
\begin{aligned}
\left|g_{n}\left(y, y^{\prime}, t, T_{1}\right)\right| \leq & C^{n}\left[\sup \left\{|b(z, s)|: y_{1}-\eta \leq z \leq y_{1}+\eta, \quad t \leq s \leq T_{1}\right\}\right]^{n+1} \\
& \cdot \frac{\left(T_{1}-t\right)^{n / 2-1 / 2}}{\varepsilon^{(n+1) / 2}} G\left(y-y^{\prime}, 2 \varepsilon\left(T_{1}-t\right)\right), \quad n=0,1,2 \ldots,
\end{aligned}
$$

for some universal constant $C$, provided $\eta \geq \sqrt{\varepsilon\left(T_{1}-t\right)}$. It follows from (3.24) that the series expansion (3.23) for the function $G$ converges provided $t \in\left[T_{1}-\Delta, T\right]$, where $\Delta$ and $\eta$ satisfy (3.20) for some sufficiently small universal $\nu>0$. In that case one has for the Green's function the estimate

$$
G\left(y, y^{\prime}, t, T_{1}\right) \leq C G\left(y-y^{\prime}, 2 \varepsilon\left(T_{1}-t\right)\right),
$$

for a universal constant $C>0$.

We can obtain estimates for the derivatives of $G$ analogous to (3.25) by differentiating the expansion (3.23) term by term. We first consider $\partial G\left(y, y^{\prime}, t, T_{1}\right) / \partial y^{\prime}$ 
for $t \in\left[T_{1}-\Delta, T_{1}\right]$ and $\Delta$ and $\eta$ satisfying (3.20). We have from (3.23) that

$$
\left|\frac{\partial g_{0}}{\partial y^{\prime}}\left(y, y^{\prime}, t, T_{1}\right)\right| \leq \frac{C}{T_{1}-t} \sqrt{\frac{\nu}{\varepsilon \Delta}} G\left(y-y^{\prime}, 2 \varepsilon\left(T_{1}-t\right)\right)
$$

for some universal constant $C$.

The integral representation in (3.23) for $\partial v_{0}\left(y, y^{\prime}, t, T_{1}\right) / \partial y^{\prime}$ gives rise to a nonintegrable singularity in the integration with respect to $s, t \leq s<T_{1}$, if we use (3.26). We therefore need to use the fact that

$$
g_{0}\left(z, y^{\prime}, s, T_{1}\right)=b(z, s) \partial K\left(z, y^{\prime}, s, T_{1}\right) / \partial z
$$

and integrate by parts with respect to $z$ in the representation $(3.23)$ for $v_{0}\left(y, y^{\prime}, t, T_{1}\right)$. We conclude that

$$
\left|\frac{\partial v_{0}}{\partial y^{\prime}}\left(y, y^{\prime}, t, T_{1}\right)\right| \leq\left[A\left(\frac{T_{1}-t}{\varepsilon}\right)^{1 / 2}+\left(\frac{\nu}{\varepsilon \Delta}\right)^{1 / 2}\right] C G\left(y-y^{\prime}, 2 \varepsilon\left(T_{1}-t\right)\right)
$$

for some universal constant $C$, where $A$ is the upper bound in (1.1) on the derivative of $b(\cdot, \cdot)$. We can use a similar method to obtain a bound on the derivative of $g_{1}$. Thus we have

$$
\left|\frac{\partial g_{1}}{\partial y^{\prime}}\left(y, y^{\prime}, t, T_{1}\right)\right| \leq\left[A\left(\frac{\nu}{\varepsilon \Delta}\right)^{1 / 2}+\frac{\nu}{\Delta \sqrt{\varepsilon\left(T_{1}-t\right)}}\right] C G\left(y-y^{\prime}, 2 \varepsilon\left(T_{1}-t\right)\right)
$$

for some universal constant $C$. Choosing $\Delta$ now to satisfy also $\Delta<\sqrt{\nu} / A$ we conclude from (3.28) and the representation (3.23) for $g_{n}$ that

$$
\left|\frac{\partial g_{n}}{\partial y^{\prime}}\left(y, y^{\prime}, t, T_{1}\right)\right| \leq \frac{\nu^{(n+1) / 2}\left(T_{1}-t\right)^{n / 2-1}}{\sqrt{\varepsilon} \Delta^{(n+1) / 2}} C^{n} G\left(y-y^{\prime}, 2 \varepsilon\left(T_{1}-t\right)\right),
$$

for $n=1,2, \ldots$, where $C$ is a universal constant. The estimate (3.29) gives an estimate on the derivatives of $v_{n}, n \geq 1$,

$$
\left|\frac{\partial v_{n}}{\partial y^{\prime}}\left(y, y^{\prime}, t, T_{1}\right)\right| \leq \frac{\nu^{(n+1) / 2}\left(T_{1}-t\right)^{n / 2}}{\sqrt{\varepsilon} \Delta^{(n+1) / 2}} C^{n} G\left(y-y^{\prime}, 2 \varepsilon\left(T_{1}-t\right)\right),
$$

for a universal constant $C$. We conclude then from (3.27) and (3.30) that upon choosing $\nu>0$ sufficiently small in a universal way, the function $G\left(y, y^{\prime}, t, T_{1}\right)$ is differentiable with respect to $y^{\prime}$ for $t \in\left[T_{1}-\Delta, T_{1}\right]$ and

$$
\left|\frac{\partial G\left(y, y^{\prime}, t, T_{1}\right)}{\partial y^{\prime}}\right| \leq \frac{C}{\sqrt{\varepsilon\left(T_{1}-t\right)}} G\left(y-y^{\prime}, 2 \varepsilon\left(T_{1}-t\right)\right),
$$

for some universal constant $C$. Hence the integral representation (3.19) is well defined for $\Delta$ and $\eta$ satisfying (3.20) and $t \in\left[T_{1}-\Delta, T_{1}\right]$.

We can obtain estimates on other derivatives of $G$ by a similar method. Observe that from (3.24) we may conclude that $G\left(y, y^{\prime}, t, T_{1}\right)$ is differentiable with respect to $y$ for $t \in\left[T_{1}-\Delta, T_{1}\right]$ and

$$
\left|\frac{\partial G\left(y, y^{\prime}, t, T_{1}\right)}{\partial y}\right| \leq \frac{C}{\sqrt{\varepsilon\left(T_{1}-t\right)}} G\left(y-y^{\prime}, 2 \varepsilon\left(T_{1}-t\right)\right),
$$


for some universal constant $C$. To obtain an estimate on $\partial^{2} G\left(y, y^{\prime}, t, T_{1}\right) / \partial y^{2}$ we must first obtain estimates on $\partial g_{n}\left(y, y^{\prime}, t, T_{1}\right) / \partial y$. Evidently we have that

$$
\left|\frac{\partial g_{0}}{\partial y}\left(y, y^{\prime}, t, T_{1}\right)\right| \leq\left[\frac{A}{\sqrt{\varepsilon\left(T_{1}-t\right)}}+\frac{\sqrt{\nu}}{\left(T_{1}-t\right) \sqrt{\varepsilon \Delta}}\right] C G\left(y-y^{\prime}, 2 \varepsilon\left(T_{1}-t\right)\right)
$$

for some universal constant $C$. To estimate $\partial g_{1}\left(y, y^{\prime}, t, T_{1}\right) / \partial y$ we write the integral representation (3.23) as an integral over $t<s<\left(T_{1}+t\right) / 2$ plus an integral over $\left(T_{1}+t\right) / 2<s<T_{1}$. Since the integral over $\left(T_{1}+t\right) / 2<s<T_{1}$ can be estimated using (3.24) we concentrate on the integral over $t<s<\left(T_{1}+t\right) / 2$. Now the kernel $\mathcal{L}_{t, y} K(y, z, t, s)$ which appears in the integral representation (3.23) for $g_{1}$ is a sum of terms generated by the boundary reflections which occur in the representation (3.21) for $G_{D}$. We consider the principle term in this series, which makes a contribution to the representation for $g_{1}$ given by

$$
\begin{aligned}
f\left(y, y^{\prime}, t, T_{1}\right)= & \int_{t}^{\left(T_{1}+t\right) / 2} d s \int_{y_{1}-\eta}^{y_{1}+\eta} d z b(y, t) \frac{\partial}{\partial y} G(y-z, \varepsilon(s-t)) g_{0}\left(z, y^{\prime}, s, T_{1}\right) \\
= & \int_{t}^{\left(T_{1}+t\right) / 2} d s \int_{y_{1}-\eta}^{y_{1}+\eta} d z b(y, t) G(y-z, \varepsilon(s-t)) \frac{\partial g_{0}}{\partial z}\left(z, y^{\prime}, s, T_{1}\right) \\
& +\int_{t}^{\left(T_{1}+t\right) / 2} d s b(y, t) G\left(y-y_{1}+\eta, \varepsilon(s-t)\right) g_{0}\left(y_{1}-\eta, y^{\prime}, s, T_{1}\right) \\
& -\int_{t}^{\left(T_{1}+t\right) / 2} d s b(y, t) G\left(y-y_{1}-\eta, \varepsilon(s-t)\right) g_{0}\left(y_{1}+\eta, y^{\prime}, s, T_{1}\right) .
\end{aligned}
$$

Denoting the first integral on the right-hand side of (3.34) by $I_{1}(y)$ we see from (3.33) that $I_{1}(y)$ is differentiable with respect to $y$ and

$$
\begin{aligned}
\left|\frac{d I_{1}}{d y}(y)\right| \leq & \frac{A\left(T_{1}-t\right)+\sqrt{\nu}\left(T_{1}-t\right)^{1 / 2}}{\sqrt{\Delta}} \\
& \cdot\left[\frac{A}{\sqrt{\varepsilon\left(T_{1}-t\right)}}+\frac{\sqrt{\nu}}{\left(T_{1}-t\right) \sqrt{\varepsilon \Delta}}\right] C G\left(y-y^{\prime}, 2 \varepsilon\left(T_{1}-t\right)\right),
\end{aligned}
$$

for some universal constant $C$. Let $I_{2}(y)$ denote the second integral on the righthand side of (3.34). Using the fact that

$$
\int_{0}^{\delta} d s \frac{\xi}{(\varepsilon s)^{3 / 2}} \exp \left[-\frac{\xi^{2}}{2 \varepsilon s}\right]=\int_{\xi^{2} /(\varepsilon \delta)}^{\infty} d z \frac{e^{-z / 2}}{\varepsilon z^{1 / 2}}
$$

we see that $I_{2}(y)$ is differentiable with respect to $y$ and

$$
\left|\frac{d I_{2}(y)}{d y}\right| \leq\left(\frac{\nu}{\varepsilon \Delta}\right)^{1 / 2}\left[A+\left\{\frac{\nu}{\Delta\left(T_{1}-t\right)}\right\}^{1 / 2}\right] C G\left(y-y^{\prime}, 2 \varepsilon\left(T_{1}-t\right)\right),
$$

for some universal constant $C$. We get an estimate similar to (3.36) for the third integral on the right-hand side of (3.34). It is clear that the higher terms in the 
series $(3.21)$ for $\mathcal{L}_{t, y} K(y, z, t, s)$ make smaller contributions to $\partial g_{1} / \partial y$ than the right-hand sides of (3.35) and (3.36). We conclude that

$$
\begin{aligned}
& \left|\frac{\partial g_{1}}{\partial y}\left(y, y^{\prime}, t, T_{1}\right)\right| \\
& \quad \leq\left(\frac{T_{1}-t}{\varepsilon}\right)^{1 / 2}\left[A+\left\{\frac{\nu}{\Delta\left(T_{1}-t\right)}\right\}^{1 / 2}\right]^{2} C G\left(y-y^{\prime}, 2 \varepsilon\left(T_{1}-t\right)\right),
\end{aligned}
$$

for some universal constant $C$. Using the representation (3.23) for $g_{n+1}$ we can now see by induction that, for $n \geq 0$,

$$
\begin{aligned}
& \left|\frac{\partial g_{n}}{\partial y}\left(y, y^{\prime}, t, T_{1}\right)\right| \\
& \quad \leq \frac{\left(T_{1}-t\right)^{n-1 / 2}}{\sqrt{\varepsilon}}\left[A+\left\{\frac{\nu}{\Delta\left(T_{1}-t\right)}\right\}^{1 / 2}\right]^{n+1} C^{n} G\left(y-y^{\prime}, 2 \varepsilon\left(T_{1}-t\right)\right),
\end{aligned}
$$

for some universal constant $C$. We can use (3.24) and (3.38) to estimate the second derivative of the function $v_{n}\left(y, y^{\prime}, t, T_{1}\right)$ in $(3.23)$ with respect to $y$. Thus we have, for $n \geq 0$,

$$
\begin{aligned}
& \left|\frac{\partial^{2} v_{n}}{\partial y^{2}}\left(y, y^{\prime}, t, T_{1}\right)\right| \\
& \quad \leq \frac{\left(T_{1}-t\right)^{n}}{\varepsilon}\left[A+\left\{\frac{\nu}{\Delta\left(T_{1}-t\right)}\right\}^{1 / 2}\right]^{n+1} C^{n} G\left(y-y^{\prime}, 2 \varepsilon\left(T_{1}-t\right)\right),
\end{aligned}
$$

for some universal constant $C$. We conclude then from (3.39) that $G\left(y, y^{\prime}, t, T_{1}\right)$ is twice differentiable with respect to $y$ for $t \in\left[T_{1}-\Delta, T_{1}\right]$ and

$$
\left|\frac{\partial^{2} G\left(y, y^{\prime}, t, T_{1}\right)}{\partial y^{2}}\right| \leq \frac{C}{\varepsilon\left(T_{1}-t\right)} G\left(y-y^{\prime}, 2 \varepsilon\left(T_{1}-t\right)\right)
$$

for some universal constant $C$.

Next we wish to estimate $\partial^{2} G\left(y, y^{\prime}, t, T_{1}\right) / \partial y \partial y^{\prime}$. We can easily obtain this from the representation (3.23) for $v_{n}$ and (3.29). Thus from (3.29) we can estimate $\partial^{2} v_{n}\left(y, y^{\prime}, t, T_{1}\right) / \partial y \partial y^{\prime}$ for $n \geq 1$. We need to integrate by parts to estimate $\partial^{2} v_{0}\left(y, y^{\prime}, t, T_{1}\right) / \partial y \partial y^{\prime}$ just as was the case for the estimate (3.27). We conclude that

$$
\left|\frac{\partial^{2} G\left(y, y^{\prime}, t, T_{1}\right)}{\partial y \partial y^{\prime}}\right| \leq \frac{C}{\varepsilon\left(T_{1}-t\right)} G\left(y-y^{\prime}, 2 \varepsilon\left(T_{1}-t\right)\right)
$$

for some universal constant $C$, provided $t \in\left[T_{1}-\Delta, T_{1}\right]$. Finally we need to estimate the derivative $\partial^{3} G\left(y, y^{\prime}, t, T_{1}\right) / \partial^{2} y \partial y^{\prime}$. To do this we must first obtain estimates on $\partial^{2} g_{n}\left(y, y^{\prime}, t, T_{1}\right) / \partial y \partial y^{\prime}$. Evidently we have that

$$
\left|\frac{\partial^{2} g_{0}\left(y, y^{\prime}, t, T_{1}\right)}{\partial y \partial y^{\prime}}\right| \leq \frac{1}{\varepsilon\left(T_{1}-t\right)}\left[A+\left\{\frac{\nu}{\Delta\left(T_{1}-t\right)}\right\}^{1 / 2}\right] C G\left(y-y^{\prime}, 2 \varepsilon\left(T_{1}-t\right)\right)
$$


for some universal constant $C$. To estimate $\partial^{2} g_{1}\left(y, y^{\prime}, t, T_{1}\right) / \partial y \partial y^{\prime}$ we write the integral representation (3.23) for $g_{1}$ as an integral over $t<s<\left(T_{1}+t\right) / 2$ plus an integral over $\left(T_{1}+t\right) / 2<s<T_{1}$. The second integral cannot be bounded by using (3.26) so we need to resort to integration by parts as we did for the estimate (3.27). To bound the contribution to $g_{1}$ from the integral over $t<s<$ $\left(T_{1}+t\right) / 2$ we use the representation (3.34). We conclude that

$$
\left|\frac{\partial^{2} g_{1}}{\partial y \partial y^{\prime}}\left(y, y^{\prime}, t, T_{1}\right)\right| \leq \frac{1}{\varepsilon}\left[A+\left\{\frac{\nu}{\Delta\left(T_{1}-t\right)}\right\}^{1 / 2}\right]^{2} C G\left(y-y^{\prime}, 2 \varepsilon\left(T_{1}-t\right)\right),
$$

for some universal constant $C$. Now by induction we see from the representation (3.23) for $g_{n}$ that, for $n \geq 0$,

$$
\begin{aligned}
& \left|\frac{\partial^{2} g_{n}}{\partial y \partial y^{\prime}}\left(y, y^{\prime}, t, T_{1}\right)\right| \\
& \quad \leq \frac{\left(T_{1}-t\right)^{n-1}}{\varepsilon}\left[A+\left\{\frac{\nu}{\Delta\left(T_{1}-t\right)}\right\}^{1 / 2}\right]^{n+1} C^{n} G\left(y-y^{\prime}, 2 \varepsilon\left(T_{1}-t\right)\right),
\end{aligned}
$$

for some universal constant $C$. Similarly to how we obtained (3.39) from (3.38) we conclude from (3.44) that, for $n \geq 0$,

$$
\begin{aligned}
& \left|\frac{\partial^{3} v_{n}}{\partial y^{2} \partial y^{\prime}}\left(y, y^{\prime}, t, T_{1}\right)\right| \\
& \quad \leq \frac{\left(T_{1}-t\right)^{n-1 / 2}}{\varepsilon^{3 / 2}}\left[A+\left\{\frac{\nu}{\Delta\left(T_{1}-t\right)}\right\}^{1 / 2}\right]^{n+1} C^{n} G\left(y-y^{\prime}, 2 \varepsilon\left(T_{1}-t\right)\right),
\end{aligned}
$$

for some universal constant $C$. We conclude then from (3.45) that provided $t \in$ $\left[T_{1}-\Delta, T_{1}\right]$, there is a universal constant $C$ such that

$$
\left|\frac{\partial^{3} G\left(y, y^{\prime}, t, T_{1}\right)}{\partial y^{2} \partial y^{\prime}}\right| \leq \frac{C}{\left[\varepsilon\left(T_{1}-t\right)\right]^{3 / 2}} G\left(y-y^{\prime}, 2 \varepsilon\left(T_{1}-t\right)\right) .
$$

We use the estimates (3.32), (3.40), (3.41), and (3.46) to obtain bounds on the derivatives in (3.17). In (3.19) we set $w(y, t)=1-u_{\varepsilon}(x, y, t)$, where the boundary functions $w_{0}, w_{-}$and $w_{+}$are all bounded by 1 . Then we estimate the derivatives of $u_{\varepsilon}(x, y, t)$ with respect to $y$ by setting $y=y_{1}$ and estimating $\partial w(y, t) / \partial y$ and $\partial^{2} w(y, t) / \partial^{2} y$ at $y=y_{1}$ using the Green's functions estimates. It is clear then that by choosing $\Delta$ to be given by its maximum value in (3.20) that we get an estimate

$$
\left|\partial u_{\varepsilon}(x, y, s) / \partial y\right|+\left|\partial^{2} u_{\varepsilon}(x, y, s) / \partial y^{2}\right| \leq C\left(\delta, y_{0}, y_{\infty}, t\right)
$$

for $(y, s)$ in any rectangle $y_{0} \leq y \leq y_{\infty}, t \leq s \leq T-\delta$. Our final task is to show that the constant $C\left(\delta, y_{0}, y_{\infty}, t\right)$ can be chosen independently of $y_{\infty}$ as $y_{\infty} \rightarrow \infty$. To see this we use the fact that the boundary functions $w_{0}, w_{-}$and $w_{+}$converge to 0 as $y_{1} \rightarrow \infty$.

Let $Y_{\varepsilon}(s)$ for $t \leq s \leq T$, be the solution of the stochastic equation (1.5) with $Y_{\varepsilon}(t)=y$, where $y>x$. We need to estimate $P\left(Y_{\varepsilon}(T)<x \mid Y_{\varepsilon}(t)=y\right)$ as $y \rightarrow \infty$. To do this we let $Z_{\varepsilon}(s)$ be the solution to the equation

$$
d Z_{\varepsilon}(s)=\left[-A Z_{\varepsilon}(s)+b(x, s)\right] d s+\sqrt{\varepsilon} d W(s), \quad s>t, \quad Z_{\varepsilon}(t)=y-x,
$$


where $A$ is the upper bound in (1.1) for the derivative of $b(\cdot, \cdot)$. Then $Y_{\varepsilon}(s) \geq$ $Z_{\varepsilon}(s)+x$ for $t \leq s \leq \tau$, where $\tau>t$ is the first hitting time at 0 for the diffusion $Z_{\varepsilon}(s)$ with $Z_{\varepsilon}(t)=y-x$. The solution to (3.48) is given by

$$
Z_{\varepsilon}(s)=(y-x) e^{-A(s-t)}+\int_{t}^{s} e^{-A\left(s-s^{\prime}\right)} b\left(x, s^{\prime}\right) d s^{\prime}+\xi_{\varepsilon}(s), \quad s>t,
$$

where $\xi_{\varepsilon}(s)$ satisfies the stochastic integral equation

$$
\xi_{\varepsilon}(s)=-A \int_{t}^{s} \xi_{\varepsilon}\left(s^{\prime}\right) d s^{\prime}+\sqrt{\varepsilon} W(s), \quad s>t .
$$

Applying Gronwall's inequality to (3.50) we have that

$$
\sup _{t \leq s \leq T}\left|\xi_{\varepsilon}(s)\right| \leq e^{A(T-t)} \sqrt{\varepsilon} \sup _{t \leq s \leq T}|W(s)| .
$$

We can estimate the probability that $\inf _{t \leq s \leq T} Z_{\varepsilon}(s)<0$ by using the inequality

$$
P\left(\sup _{t \leq s \leq T}|W(s)|>a\right) \leq\left[\frac{8(T-t)}{\pi a^{2}}\right]^{1 / 2} \exp \left[-\frac{a^{2}}{2(T-t)}\right] .
$$

Let us assume that the second term on the right-hand side of (3.49) is smaller in absolute value than half the first term for $t \leq s \leq T$. This can evidently be accomplished by choosing $y-x$ sufficiently large. Then from (3.51) and (3.52) we conclude that

$$
\begin{aligned}
& P\left(\inf _{t \leq s \leq T} Z_{\varepsilon}(s)<0\right) \\
& \quad \leq\left[\frac{8(T-t)}{\pi}\right]^{1 / 2} \frac{2 \sqrt{\varepsilon} e^{2 A(T-t)}}{(y-x)} \exp \left[-\frac{(y-x)^{2}}{8 \varepsilon(T-t)} e^{-4 A(T-t)}\right] .
\end{aligned}
$$

Using the inequality

$$
P\left(Y_{\varepsilon}(T)<x \mid Y_{\varepsilon}(t)=y\right) \leq P\left(\inf _{t \leq s \leq T} Z_{\varepsilon}(s)<0\right)
$$

we obtain from (3.53) bounds on the boundary functions $w_{0}, w_{-}$and $w_{+}$in (3.19). Evidently these decay exponentially in $y_{1}$ as $y_{1} \rightarrow \infty$, whereas it follows from (3.20) and the Lipschitz condition $(1.1)$ on $b(\cdot, \cdot)$ that we may take $\Delta \sim 1 / y_{1}^{2}$ as $y_{1} \rightarrow \infty$. We conclude that (3.47) holds uniformly as $y_{\infty} \rightarrow \infty$.

Lemma 3.5. Suppose $b(\cdot, \cdot)$ satisfies (1.1). Then for $x, y \in \mathbb{R}, t<T$, and $\varepsilon<1$ the inequality

$$
q_{\varepsilon}(x, y, t) \geq q(x, y, t)-C(x, y, t, T) \sqrt{\varepsilon}
$$

holds, where $q(x, y, t)$ is given by (1.10) and $C(x, y, t, T)$ is a constant independent of $\varepsilon$. 
Proof. Suppose $y_{0}<x$ and $y>y_{0}$. Then by Lemma 3.4 we have the representation

$$
\begin{aligned}
q_{\varepsilon}(x, y, t)=E\{ & \frac{1}{2} \int_{t}^{(T-\delta) \wedge \tau}\left[\lambda_{\varepsilon}\left(y_{\varepsilon}(s), s\right)-b\left(y_{\varepsilon}(s), s\right)\right]^{2} d s \\
& \left.+q_{\varepsilon}\left(x, y_{\varepsilon}((T-\delta) \wedge \tau),(T-\delta) \wedge \tau\right) \mid y_{\varepsilon}(t)=y\right\} .
\end{aligned}
$$

Here $\lambda_{\varepsilon}(y, s)$ is given by the formula

$$
\lambda_{\varepsilon}(y, s)=b(y, s)-\partial q_{\varepsilon}(x, y, s) / \partial y, \quad y \in \mathbb{R}, s<T .
$$

By Lemma 3.4 the function $\lambda_{\varepsilon}(y, s)$ is uniformly Lipschitz in $y$ for $y \geq y_{0}$ and $t \leq s \leq T-\delta$. Hence (1.14) has a unique solution $y_{\varepsilon}(s)$ for $t \leq s \leq(T-\delta) \wedge \tau$, where $\tau$ is the first hitting time at $y_{0}$.

We consider a random path $y_{\varepsilon}(s)$ for $t \leq s \leq T-\delta$, for which $\tau>T-\delta$, and associate with it a classical path $y_{\varepsilon, c}(s)$ for $t \leq s \leq T$. To do this let $k$ be defined by

$$
k=\max \left[x-\delta-y-\int_{t}^{T-\delta} \lambda_{\varepsilon}\left(y_{\varepsilon}(s), s\right) d s, 0\right] .
$$

Then $y_{\varepsilon, c}(s)$ for $t \leq s \leq T$ is the solution to the initial value problem

$$
\begin{cases}\frac{d y_{\varepsilon, c}(s)}{d s}=\lambda_{\varepsilon}\left(y_{\varepsilon}(s), s\right)+k /(T-t-\delta), & t \leq s \leq T-\delta \\ \frac{d y_{\varepsilon, c}(s)}{d s}=2-b(x, s)+b\left(y_{\varepsilon, c}(s), s\right), & T-\delta \leq s \leq T, y_{\varepsilon, c}(t)=y\end{cases}
$$

Since from $(3.57)$ one has that $y_{\varepsilon, c}(T-\delta) \geq x-\delta$, it follows that $y_{\varepsilon, c}(T) \geq x$ provided $\delta$ is sufficiently small. Hence from (1.10) we conclude that

$$
\frac{1}{2} \int_{t}^{T}\left[\frac{d y_{\varepsilon, c}(s)}{d s}-b\left(y_{\varepsilon, c}(s), s\right)\right]^{2} d s \geq q(x, y, t) .
$$

From (1.14) and (3.58) we see that

$$
y_{\varepsilon, c}(s)-y_{\varepsilon}(s)=\frac{k(s-t)}{(T-t-\delta)}+\sqrt{\varepsilon}[W(s)-W(t)], \quad t \leq s \leq T-\delta .
$$

We may also rewrite the parameter $k$ in (3.57) as

$$
k=\max \left[x-\delta-y_{\varepsilon}(T-\delta)+\sqrt{\varepsilon}[W(T-\delta)-W(t)], 0\right] .
$$

Observe now that

$$
\frac{1}{2} \int_{t}^{(T-\delta)}\left[\lambda_{\varepsilon}\left(y_{\varepsilon}(s), s\right)-b\left(y_{\varepsilon}(s), s\right)\right]^{2} d s
$$

$$
\begin{aligned}
\geq & \frac{1}{2} \int_{t}^{(T-\delta)}\left[\frac{d y_{\varepsilon, c}(s)}{d s}-b\left(y_{\varepsilon, c}(s), s\right)\right]^{2} d s \\
& -\int_{t}^{(T-\delta)}\left|\frac{d y_{\varepsilon, c}(s)}{d s}-b\left(y_{\varepsilon, c}(s), s\right)\right| \\
& \cdot\left|b\left(y_{\varepsilon, c}(s), s\right)-b\left(y_{\varepsilon}(s), s\right)-k /(T-t-\delta)\right| d s .
\end{aligned}
$$


Evidently from (3.59) the first term on the right-hand side of (3.62) is bounded below by $q(x, y, t)-C \delta$ for some constant $C$. Using (3.61) and Lemma 3.1 we may bound the second term on the right-hand side of (3.62). First observe that this second term is bounded in absolute value by

$$
\begin{aligned}
& \frac{\eta}{2} \int_{t}^{(T-\delta)}\left[\lambda_{\varepsilon}\left(y_{\varepsilon}(s), s\right)-b\left(y_{\varepsilon}(s), s\right)\right]^{2} d s \\
& \quad+\left[1+\frac{1}{2 \eta}\right] \int_{t}^{(T-\delta)}\left[b\left(y_{\varepsilon, c}(s), s\right)-b\left(y_{\varepsilon}(s), s\right)-k /(T-t-\delta)\right]^{2} d s
\end{aligned}
$$

for any $\eta>0$. From (3.60) and the Lipschitz condition $(1.1)$ on $b(\cdot, \cdot)$, the second term in (3.63) is bounded from above as

$$
\begin{aligned}
& \int_{t}^{(T-\delta)}\left[b\left(y_{\varepsilon, c}(s), s\right)-\right.\left.b\left(y_{\varepsilon}(s), s\right)-k /(T-t-\delta)\right]^{2} d s \\
& \leq C_{1} \varepsilon \int_{t}^{T-\delta}[W(s)-W(t)]^{2} d s+C_{2} k^{2}
\end{aligned}
$$

where the constants $C_{1}$ and $C_{2}$ depend only on $T-t$, assuming $\delta<(T-t) / 2$. Hence from (3.61), (3.63), and (3.64) we conclude that the second term on the right-hand side of (3.62) is bounded by

$$
\begin{gathered}
\frac{\eta}{2} \int_{t}^{(T-\delta)}\left[\lambda_{\varepsilon}\left(y_{\varepsilon}(s), s\right)-b\left(y_{\varepsilon}(s), s\right)\right]^{2} d s+\frac{C_{1} \varepsilon}{\eta} \int_{t}^{(T-\delta)}[W(s)-W(t)]^{2} d s \\
+\frac{C_{2} \varepsilon}{\eta}[W(T-\delta)-W(t)]^{2}+\frac{C_{3}}{\eta}\left\{\max \left[x-\delta-y_{\varepsilon}(T-\delta), 0\right]\right\}^{2}
\end{gathered}
$$

for any $\eta, 0<\eta<1$, and constants $C_{1}, C_{2}$, and $C_{3}$ depending only on $T-t$. It follows then on taking $\eta \sim \delta$ in (3.65) and using Lemma 3.1 that

$$
\begin{aligned}
& q_{\varepsilon}\left(x, y_{\varepsilon}(T-\delta), T-\delta\right)+\frac{1}{2} \int_{t}^{(T-\delta)}\left[\lambda_{\varepsilon}\left(y_{\varepsilon}(s), s\right)-b\left(y_{\varepsilon}(s), s\right)\right]^{2} d s \\
& \quad \geq q(x, y, t)-C_{1} \delta-\frac{C_{2} \varepsilon}{\delta} \int_{t}^{(T-\delta)}[W(s)-W(t)]^{2} d s+O(\varepsilon) \\
& -\frac{C_{3} \varepsilon}{\delta}[W(T-\delta)-W(t)]^{2}-C_{4} \delta \int_{t}^{(T-\delta)}\left[\lambda_{\varepsilon}\left(y_{\varepsilon}(s), s\right)-b\left(y_{\varepsilon}(s), s\right)\right]^{2} d s,
\end{aligned}
$$

for constants $C_{1}, C_{2}, C_{3}$, and $C_{4}$ depending only on $T-t$.

To conclude the proof we take the expectation of (3.66) on a set of paths $y_{\varepsilon}(s), t \leq s \leq T-\delta$, for which $\tau>T-\delta$. To find a suitable set of paths note that $\partial q_{\varepsilon}(x, y, s) / \partial y \leq 0$ for $y \in \mathbb{R}$ and $s<T$, whence (3.56) implies that $\lambda_{\varepsilon}(y, s) \geq b(y, s), y \in \mathbb{R}, s<T$. Thus $y_{\varepsilon}(s) \geq Y_{\varepsilon}(s)$ for $t \leq s \leq T-\delta$, where $Y_{\varepsilon}(s)$ is the solution to (1.5) with $Y_{\varepsilon}(t)=y$. We have already estimated the fluctuation of $Y_{\varepsilon}(s)$ for $t \leq s \leq T$, from $y$ by (3.4). We therefore conclude that for given $y$ we may choose $y_{0}<y$ such that

$$
\sup _{t \leq s \leq T}|W(s)-W(t)|<1 / \sqrt{\varepsilon} \text { implies } \tau>T-\delta .
$$


The inequality (3.54) follows now upon taking $\delta=\sqrt{\varepsilon}$ in (3.66) and taking the expectation on the paths for which (3.67) holds.

Proof of Theorem 1.1. Evidently (1.12) follows from Lemmas 3.3 and 3.5.

\section{The optimally controlled process}

In Lemma 3.5 we already used the optimally controlled process $y_{\varepsilon}(s)$ of $(1.14)$ with controller (1.16) to obtain a lower bound on $q_{\varepsilon}(x, y, t)$. The main goal of this section is to prove that $\liminf _{s \rightarrow T} y_{\varepsilon}(s)>x$ with probability 1 . To do this we need to prove some short time asymptotic results for the cost function $q_{\varepsilon}(x, y, t)$.

Lemma 4.1. Suppose that $0<T-t<\delta \leq \varepsilon<1$. Then the function $q_{\varepsilon}(x, y, t)$ satisfies the inequalities

$$
\begin{aligned}
0<q_{\varepsilon}(x, y, t) \leq & C \varepsilon+(x-y)^{2} /(T-t) \\
& +C(x, \delta)\left[(y-x)^{2}+|y-x|+\sqrt{\varepsilon(T-t)}\right], \quad y<x, \\
0<q_{\varepsilon}(x, y, t) \leq & C \varepsilon \exp \left[-(x-y)^{2} / 2 \varepsilon(T-t)\right] \\
& +C(x, \delta)\left[(y-x)^{2}+|y-x|+\sqrt{\varepsilon(T-t)}\right], \quad y>x,
\end{aligned}
$$

where $C$ is a universal constant and $C(x, \delta)$ depends only on $x$ and $\delta$. The function $\partial q_{\varepsilon}(x, y, t) / \partial y$ satisfies the inequality

$$
\begin{aligned}
&-\frac{\partial q_{\varepsilon}}{\partial y}(x, y, t) \geq\left(\frac{x-y}{T-t}\right) \cdot \exp [-C(x, \delta)\{(T-t)|\log (T-t)| \\
&\left.\left.+[(T-t) / \varepsilon]^{1 / 2}+(y-x)^{2} / \varepsilon+|y-x| / \varepsilon\right\}\right]
\end{aligned}
$$

for a constant $C(x, \delta)$ depending only on $x$ and $\delta$.

Proof. We apply the Schwarz inequality to the PDE (1.8) for $q_{\varepsilon}(x, y, t)$. Thus for any $\alpha>0$,

$$
\frac{\partial q_{\varepsilon}}{\partial t}+b(x, t) \frac{\partial q_{\varepsilon}}{\partial y}-\frac{1}{2}(1-\alpha)\left(\frac{\partial q_{\varepsilon}}{\partial y}\right)^{2}+\frac{\varepsilon}{2} \frac{\partial^{2} q_{\varepsilon}}{\partial y^{2}}+\frac{1}{2 \alpha}[b(y, t)-b(x, t)]^{2} \geq 0
$$

Setting $v_{\alpha}(y, t)=\exp \left[-(1-\alpha) q_{\varepsilon}(x, y, t) / \varepsilon\right]$, we see from (4.3) that

$$
\frac{\partial v_{\alpha}}{\partial t}+b(x, t) \frac{\partial v_{\alpha}}{\partial y}+\frac{\varepsilon}{2} \frac{\partial^{2} v_{\alpha}}{\partial y^{2}} \leq \frac{(1-\alpha)}{2 \alpha \varepsilon}[b(y, t)-b(x, t)]^{2} v_{\alpha},
$$

provided $\alpha<1$. It follows now from (4.4) that $v_{\alpha}$ is bounded below by

$$
\begin{aligned}
& v_{\alpha}(y, t) \geq E\left[v_{\alpha}(y+g(T)+\sqrt{\varepsilon} W(T-t), T)\right. \\
& \left.\cdot \exp \left\{-\int_{t}^{T}(1-\alpha)[b(y+g(s)+\sqrt{\varepsilon} W(s-t), s)-b(x, s)]^{2} d s / 2 \alpha \varepsilon\right\}\right],
\end{aligned}
$$


where $W(\cdot)$ is Brownian motion and $g(\cdot)$ is given by

$$
g(s)=\int_{t}^{s} b\left(x, s^{\prime}\right) d s^{\prime}, \quad t \leq s \leq T .
$$

Observing that $v_{\alpha}$ has terminal data $v_{\alpha}(y, T)=0$ for $y<x$, and $v_{\alpha}(y, T)=1$ for $y>x$, we conclude from (4.5) that

$$
v_{\alpha}(y, t) \geq \int_{x-g(T)}^{\infty} \frac{1}{\sqrt{2 \pi \varepsilon(T-t)}} \exp \left[-\frac{(y-z)^{2}}{2 \varepsilon(T-t)}\right] F(y, z) d z,
$$

where $F(y, z)$ is given by the formula

$$
\begin{aligned}
& F(y, z)=E\left[\operatorname { e x p } \left\{-\int_{t}^{T}(1-\alpha)[b([(T-s) y+(s-t) z] /(T-t)\right.\right. \\
& \left.\left.+\sqrt{\varepsilon}[W(s-t)-(s-t) W(T-t) /(T-t)]+g(s), s)-b(x, s)]^{2} d s / 2 \alpha \varepsilon\right\}\right] .
\end{aligned}
$$

In (4.7) we have used the Brownian bridge representation for Brownian motion conditioned at times $t$ and $T$. Using Jensen's inequality in (4.8) and the Lipschitz bound $(1.1)$ on $b(\cdot, \cdot)$, we conclude that

$$
\begin{aligned}
-\log F(y, z) \leq & \frac{A^{2}(1-\alpha)}{2 \alpha \varepsilon} \int_{t}^{T} d s E[\{(T-s)(y-x)+(s-t)(z-x)] /(T-t) \\
& \left.+g(s)+\sqrt{\varepsilon}[W(s-t)-(s-t) W(T-t) /(T-t)]\}^{2}\right] \\
= & \frac{A^{2}(1-\alpha)}{2 \alpha \varepsilon} \int_{t}^{T} d s\{[(T-s)(y-x)+(s-t)(z-x)] /(T-t)+g(s)\}^{2} \\
& +\frac{A^{2}(1-\alpha)}{2 \alpha} \int_{t}^{T} d s(s-t)(T-s) /(T-t) .
\end{aligned}
$$

It follows now from (4.6) and (4.9) that for any $\delta>0$ there is a constant $C(x, \delta)$ depending only on $x$ and $\delta$ such that

$$
\begin{aligned}
-\log F(y, z) \leq \frac{A^{2}(1-\alpha)}{2 \alpha \varepsilon} & {\left[(z-x)^{2}(T-t)+(y-x)^{2}(T-t)\right.} \\
+ & \left.C(x, \delta)(T-t)^{3}+\varepsilon(T-t)^{2} / 6\right], \quad T-t<\delta .
\end{aligned}
$$

We may combine (4.7) and (4.10) to obtain an upper bound on $q_{\varepsilon}(x, y, t)$. Thus on using the inequality $(z-x)^{2} \leq 2(z-y)^{2}+2(y-x)^{2}$ in (4.10), we conclude from (4.7) that for $T-t<\delta$,

$$
\begin{aligned}
& v_{\alpha}(y, t) \geq \exp \left[-\frac{A^{2}(1-\alpha)}{2 \alpha \varepsilon}\left\{3(y-x)^{2}(T-t)+C(x, \delta)(T-t)^{3}+\varepsilon(T-t)^{2} / 6\right\}\right] \\
& (4.11) \quad \cdot \int_{x-y-g(T)}^{\infty} \frac{1}{\sqrt{2 \pi \varepsilon(T-t)}} \exp \left[-z^{\prime 2}\left\{\frac{1}{2 \varepsilon(T-t)}+\frac{A^{2}(1-\alpha)}{\alpha \varepsilon}(T-t)\right\}\right] d z^{\prime} .
\end{aligned}
$$


Let us recall the inequality

$$
\frac{1}{a}\left(1-\frac{1}{a^{2}}\right) e^{-a^{2} / 2}<\int_{a}^{\infty} e^{-z^{2} / 2} d z<\frac{1}{a} e^{-a^{2} / 2}, \quad a>0 .
$$

We shall use it to show that there is a universal constant $C$ such that

$$
\int_{a+\eta}^{\infty} e^{-z^{2} / 2} d z \geq \exp \left[-\eta^{2} / 2-C \eta \max \{a, 1\}\right] \int_{a}^{\infty} e^{-z^{2} / 2} d z, \quad \eta>0, a \in \mathbb{R}
$$

To see this observe that, by Jensen's inequality,

$$
\int_{a+\eta}^{\infty} e^{-z^{2} / 2} d z \geq \exp \left[-\eta^{2} / 2-\eta\langle Z\rangle\right] \int_{a}^{\infty} e^{-z^{2} / 2} d z
$$

where $Z$ is the standard normal variable conditioned on $Z>a$. Evidently if $a \leq 2$ then $|\langle Z\rangle| \leq C_{1}$ for some universal constant $C_{1}$. If $a \geq 2$ we see from (4.12) that

$$
\langle Z\rangle \leq a\left(1-\frac{1}{a^{2}}\right)^{-1} \leq \frac{4}{3} a,
$$

whence (4.13) holds for all $a \in \mathbb{R}$.

We shall apply the inequality (4.13) in (4.11) to obtain an upper bound on $q_{\varepsilon}(x, y, t)$ in terms of the cumulative distribution function $\Phi$ for the standard normal variable. Now the integral with respect to $z^{\prime}$ on the right-hand side of (4.11) is given by

$$
\frac{1}{\left[1+2 A^{2}(1-\alpha)(T-t)\right]^{1 / 2}} \Phi\left(\frac{y-x+g(T)}{\sqrt{\varepsilon(T-t)}}\left[1+2 A^{2}(1-\alpha)(T-t)\right]^{1 / 2}\right)
$$

if we set $\alpha=T-t$. We write the argument of $\Phi$ in (4.14) as $-[a+\eta]$ with $a=(x-y) / \sqrt{\varepsilon(T-t)}$ and apply (4.13). Thus for $T-t<\delta \leq \varepsilon$, we obtain the inequality

$$
\begin{aligned}
& \Phi\left(\frac{y-x+g(T)}{\sqrt{\varepsilon(T-t)}}\left[1+2 A^{2}(1-\alpha)(T-t)\right]^{1 / 2}\right) \\
& \geq \Phi\left(\frac{y-x}{\sqrt{\varepsilon(T-t)}}\right) \exp \left[-\frac{C(x, \delta)}{\varepsilon}\left\{(y-x)^{2}+|y-x|+\sqrt{\varepsilon(T-t)}\right\}\right]
\end{aligned}
$$

for some constant $C(x, \delta)$ depending only on $x$ and $\delta$. If we combine (4.15) with (4.11), taking $\alpha=T-t$, we obtain for $q_{\varepsilon}$ the upper bound

$$
\begin{aligned}
q_{\varepsilon}(x, y, t) \leq & -\varepsilon \log \Phi([y-x] / \sqrt{\varepsilon(T-t)}) \\
& +C(x, \delta)\left[(y-x)^{2}+|y-x|+\sqrt{\varepsilon(T-t)}\right], \quad T-t<\delta \leq \varepsilon
\end{aligned}
$$

for a constant $C(x, \delta)$ depending only on $x$ and $\delta$. The inequality (4.1) follows from (4.16) upon using (4.12). Note that (4.12) for $y<x$ follows from (4.16) upon using the fact that $\log a \leq a^{2} / 2$ for $a>1$. 
Next we turn to estimating $\partial q_{\varepsilon}(x, y, t) / \partial y$. To do this we consider the Green's function $G\left(y, y^{\prime}, t, T\right)$ of (1.4). It follows from (1.4) that

$$
-\partial u_{\varepsilon}(x, y, t) / \partial x=G(y, x, t, T) .
$$

If we differentiate (1.2) with respect to $y$ and use the maximum principle, we see also that

$$
\partial u_{\varepsilon}(x, y, t) / \partial y \geq e^{-A(T-t)} G(y, x, t, T),
$$

where $A$ is the Lipschitz constant in (1.1). Since

$$
-\partial q_{\varepsilon}(x, y, t) / \partial y=\varepsilon\left[\partial u_{\varepsilon}(x, y, t) / \partial y\right] / u_{\varepsilon}(x, y, t),
$$

we may obtain the lower bound (4.2) by finding a lower bound for $G(y, x, t, T)$ and a lower bound for $q_{\varepsilon}(x, y, t)$ which is complimentary to (4.16).

We turn to the problem of obtaining a lower bound for $q_{\varepsilon}$. Instead of (4.3) we use the differential inequality

$$
\frac{\partial q_{\varepsilon}}{\partial t}+b(x, t) \frac{\partial q_{\varepsilon}}{\partial y}-\frac{1}{2}(1+\alpha)\left(\frac{\partial q_{\varepsilon}}{\partial y}\right)^{2}+\frac{\varepsilon}{2} \frac{\partial^{2} q_{\varepsilon}}{\partial y^{2}}-[b(y, t)-b(x, t)]^{2} / 2 \alpha \leq 0
$$

for any $\alpha>0$. Setting $v_{\alpha}(y, t)=\exp \left[-(1+\alpha) q_{\varepsilon}(x, y, t) / \varepsilon\right]$ we see from (4.18) that

$$
v_{\alpha}(y, t) \leq \int_{x-g(T)}^{\infty} \frac{1}{\sqrt{2 \pi \varepsilon(T-t)}} \exp \left[-\frac{(y-z)^{2}}{2 \varepsilon(T-t)}\right] F(y, z) d z,
$$

where $F(y, z)$ is given by the formula

$$
\begin{aligned}
F(y, z)= & E\left[\operatorname { e x p } \left\{\int_{t}^{T} \frac{A^{2}(1+\alpha)}{2 \alpha \varepsilon} d s([T-s)(y-x)+(s-t)(z-x)] /(T-t)\right.\right. \\
& \left.\left.+g(s)+\sqrt{\varepsilon}[W(s-t)-(s-t) W(T-t) /(T-t)])^{2}\right\}\right] .
\end{aligned}
$$

The expectation in (4.20) cannot be evaluated exactly as was the case with (4.9), but it may be estimated using the fact that one knows the probability density function of $\sup _{t \leq s \leq T} W(s-t)$. Taking $\alpha=T-t$ in (4.20), we see from this that, for $T-t<\delta$,

$$
\log F(y, z) \leq \frac{C A^{2}}{\varepsilon}\left[(z-x)^{2}+(y-x)^{2}+C(x, \delta)(T-t)^{2}+\varepsilon(T-t)\right],
$$

for a universal constant $C$ and a constant $C(x, \delta)$ depending on only $x$ and $\delta$. Note here that we require $\delta<1 / A^{2}$ for the expectation (4.20) to be finite. To obtain the lower bound on $q_{\varepsilon}$ we combine (4.21) and (4.19) with the inequality (4.13). Since we are obtaining an upper bound on the function $v_{\alpha}(y, t)$, we apply $(4.13)$ with $a+\eta=(x-y) / \sqrt{\varepsilon(T-t)}$. Hence we get an inequality

$$
\begin{aligned}
q_{\varepsilon}(x, y, t) \geq & -\varepsilon \log \Phi\left(\frac{y-x}{\sqrt{\varepsilon(T-t)}}\right) \\
& -C(x, \delta)\left[(y-x)^{2}+|y-x|+\sqrt{\varepsilon(T-t)}\right], \quad T-t<\delta \leq \varepsilon,
\end{aligned}
$$

complimentary to $(4.16)$, for a constant $C(x, \delta)$ depending only on $x$ and $\delta$. 
The lower bound for $G(y, x, t, T)$ may be obtained in a similar way to the upper bound on $q_{\varepsilon}(x, y, t)$. Let $0<\Delta<T-t$ and $0<\alpha<1$. Then, just as in (4.5), we have that

$$
\begin{aligned}
& G(y, x, t, T)^{1-\alpha} \geq \int_{-\infty}^{\infty} \frac{1}{\sqrt{2 \pi \varepsilon(T-t-\Delta)}} \\
& \cdot \exp \left[-\frac{(y-z)^{2}}{2 \varepsilon(T-t-\Delta)}\right] F_{\Delta}(y, z) G(z+g(T-\Delta), x, T-\Delta, T)^{1-\alpha} d z
\end{aligned}
$$

where $F_{\Delta}$ is as in (4.8) but with $T$ replaced by $T-\Delta$. Observe that we cannot take $\Delta \rightarrow 0$ on the right-hand side of (4.23) since the integrand would contain in the limit $\delta(z+g(T-\Delta)-x)^{1-\alpha}$, which is identically zero. We shall choose $\Delta$ so that $0<\Delta<<T-t$ and $\alpha=T-t$, in a way that the function $z \rightarrow G(z+$ $g(T-\Delta), x, T-\Delta, T)^{1-\alpha}$ is approximately a Dirac delta function concentrated at $x-g(T-\Delta)$.

It is evident that the right-hand side of (4.23) is decreased upon replacing $G$ by the corresponding Dirichlet Green's function $G_{D}$ for an interval centered at $x$. As in Lemma 3.4 we choose this interval sufficiently small and $\Delta$ sufficiently small so that $G_{D}$ may be expanded in a perturbation series. The condition for this has already been given in (3.20). Thus the Green's function $G_{D}(z, x, T-\Delta, T)$ on the interval $x-\eta \leq z \leq x+\eta$ has a convergent perturbation expansion provided $\eta$ and $\Delta$ satisfy the inequalities

$$
\varepsilon \Delta \leq \eta^{2}, \quad \Delta \leq \frac{\nu \varepsilon}{[A \eta+C(x, \delta)]^{2}}, \quad \Delta \leq \delta,
$$

where $A$ is the Lipschitz constant from (1.1) and $C(x, \delta)$ is a constant depending only on $x$ and $\delta$. In this case there are universal constants $C_{1}$ and $C_{2}$ such that

$$
\begin{aligned}
\int_{x-\eta}^{x+\eta} G_{D}(z, x, T-\Delta, T) d z \geq & 1-C_{2} \exp \left[-\eta^{2} / 4 \varepsilon \Delta\right] \\
& -C_{2}[A \eta+C(x, \delta)](\Delta / \varepsilon)^{1 / 2}, \quad 0<\Delta<\delta .
\end{aligned}
$$

Observe that if we take $\Delta=(T-t)^{3}$ and $\eta=(T-t) \sqrt{\varepsilon}$ then the right-hand side of (4.25) is bounded below by $1-C(x, \delta)(T-t)$ for $0<T-t<\delta \leq \varepsilon$, where $C(x, \delta)$ depends only on $x$ and $\delta$. Taking $\alpha=T-t$ we may see further that with the same values for $\Delta$ and $\eta$ the inequality

$$
\int_{x-\eta}^{x+\eta} G_{D}(z, x, T-\Delta, T)^{1-\alpha} d z \geq 1-C(x, \delta)(T-t)|\log (T-t)|,
$$

$0<T-t<\delta \leq \varepsilon$, holds for a constant $C(x, \delta)$ depending only on $x$ and $\delta$. It follows then from (4.10), (4.23), and (4.26) that

$$
\begin{aligned}
G(y, x, t, T) \geq & \frac{1}{\sqrt{2 \pi \varepsilon(T-t)}} \exp \left[-\frac{(y-x)^{2}}{2 \varepsilon(T-t)}-C(x, \delta)\{(T-t)|\log (T-t)|\right. \\
& \left.\left.+(y-27) \quad+x)^{2} / \varepsilon+|y-x| / \varepsilon\right\}\right], \quad 0<T-t<\delta \leq \varepsilon,
\end{aligned}
$$

for a constant $C(x, \delta)$ depending only on $x$ and $\delta$. 
To obtain the lower bound (4.2) we combine (4.22) and (4.27) using (4.17). The inequality (4.2) now follows from (4.12).

Remark 4.2. There is a vast literature on short time asymptotics of solutions to diffusive equations. See in particular the classical papers of Kannai [11], Minakshisundaram [17], Molchanov [18], and Varadhan [22].

Lemma 4.1 shows that for $y<x$ and $s<T$ with $T-s$ small, the optimal controller $\lambda^{*}(x, y, s)$, given by (1.16) for the stochastic control problem (1.15), is approximately $\lambda^{*}(x, y, s)=(x-y) /(T-s)$. This will enable us to show that the solution $y_{\varepsilon}(s)$ of the corresponding stochastic differential equation (1.14) satisfies $\liminf _{s \rightarrow T} y_{\varepsilon}(s)>x$ with probability 1 . First we show this for the linear approximation which we have just established.

Lemma 4.3. Suppose $\mu>0$ and $\varepsilon>0$, and that $Z_{\varepsilon}(s)$, for $t \leq s<T$, is a solution to the SDE

$$
d Z_{\varepsilon}(s)=\frac{-\mu Z(s)}{T-s} d s+\sqrt{\varepsilon} d W(s)
$$

with initial condition $Z_{\varepsilon}(t)=z \in \mathbb{R}$. Then $\lim _{s \rightarrow T} Z_{\varepsilon}(s)=0$ with probability 1 , and if $\mu>1 / 2$ then $\liminf _{s \rightarrow T} Z_{\varepsilon}(s) / \sqrt{T-s}=-\infty$ with probability 1 .

Proof. The SDE (4.28) is explicitly solvable, whence we find

$$
Z_{\varepsilon}(s)=\left(\frac{T-s}{T-t}\right)^{\mu} z+\sqrt{\varepsilon} \int_{t}^{s}\left(\frac{T-s}{T-s^{\prime}}\right)^{\mu} d W\left(s^{\prime}\right), \quad t \leq s<T .
$$

Thus $Z_{\varepsilon}(s)$ is a Gaussian variable with mean of order $(T-s)^{\mu}$ as $s \rightarrow T$. We shall assume without loss of generality that $\mu>1 / 2$, in which case the variance of $Z_{\varepsilon}(s)$ has order $T-s$ as $s \rightarrow T$. Hence the standard deviation of $Z_{\varepsilon}(s)$ dominates the mean for $s \rightarrow T$. For $n=0,1,2, \ldots$, let $s_{n}=T-(T-t) / 2^{n}$, so $t=s_{0}<s_{1}<$ $s_{2}<\cdots<T$. For $t<s<T$ we consider the martingale $M(s)$ defined by

$$
M(s)=\int_{t}^{s}\left(T-s^{\prime}\right)^{-\mu} d W\left(s^{\prime}\right),
$$

which by Doob's inequality satisfies

$$
P\left(\sup _{t \leq s \leq s_{n}}|M(s)|>a\right) \leq \frac{2^{(2 \mu-1) n}}{a^{2}(2 \mu-1)(T-t)^{2 \mu-1}}, \quad a>0 .
$$

It follows that

$$
\sum_{n=1}^{\infty} P\left(\sup _{t \leq s \leq s_{n}}|M(s)|>2^{(\mu-1 / 4) n}\right)<\infty .
$$

Hence, by the Borel-Cantelli lemma, $\limsup _{s \rightarrow T}(T-s)^{\mu}|M(s)|=0$ with probability 1 . We conclude from (4.29) that $\lim _{s \rightarrow T} Z_{\varepsilon}(s)=0$ with probability 1 . 
We turn to showing that $\liminf _{s \rightarrow T} Z_{\varepsilon}(s) / \sqrt{T-s}=-\infty$ with probability 1. For a positive integer $n$ we define variables $Y_{n}$ by

$$
Y_{n}=\left(T-s_{n}\right)^{\mu-1 / 2}\left[M\left(s_{n}\right)-M\left(s_{n-1}\right)\right] .
$$

We may write the $Z_{\varepsilon}\left(s_{n}\right)$ in terms of the $Y_{n}$ as

$$
Z_{\varepsilon}\left(s_{n}\right)=\left(\frac{T-s_{n}}{T-t}\right)^{\mu} z+\sqrt{\varepsilon\left(T-s_{n}\right)} \sum_{m=1}^{n} Y_{m} / 2^{(n-m)(\mu-1 / 2)}, \quad n=1,2, \ldots
$$

Evidently the $Y_{n}, n \geq 1$, are independent and Gaussian with zero mean and variance $\operatorname{var}\left(Y_{n}\right)=\left[1-2^{1-2 \mu}\right] /(2 \mu-1)$. By the Borel-Cantelli lemma, for any $K>0$ one has $Y_{n}<-K$ for infinitely many $n$, with probability 1 . Thus if in (4.30) we were to replace the sum over $1 \leq m \leq n$ by its dominant term $m=n$, we would have shown that $\liminf _{n \rightarrow \infty} Z_{\varepsilon}(s) / \sqrt{T-s_{n}}=-\infty$ with probability 1 .

To take account of the sum in (4.30) we need to make a more elaborate argument. Denoting the sum in (4.30) by $\xi_{n}$ it is easy to see that

$$
\xi_{n}=Y_{n}+\xi_{n-1} / 2^{(\mu-1 / 2)}, \quad n \geq 1,
$$

where $\xi_{0}=0$. For $\xi \in \mathbb{R}$ and $n \geq 1$, we put

$$
u(\xi, n)=P\left[\xi_{m}>a, 1 \leq m \leq n \mid \xi_{0}=\xi\right],
$$

where the $\xi_{n}$ are defined by the recurrence (4.31). Setting $\delta=1 / 2^{(\mu-1 / 2)}<1$, it is easy to see that the $u(\xi, n)$ satisfy the recurrence equation

$$
u(\xi, n)=\frac{1}{\sqrt{2 \pi \sigma^{2}}} \int_{a}^{\infty} d \xi^{\prime} u\left(\xi^{\prime}, n-1\right) \exp \left[-\left(\xi^{\prime}-\delta \xi\right)^{2} / 2 \sigma^{2}\right], \quad n \geq 1,
$$

where we define $u(\xi, 0)=1, \xi \in \mathbb{R}$, and $\sigma^{2}=\left[1-2^{1-2 \mu}\right] /(2 \mu-1)$. If, for $z>0, \hat{u}(\xi, z)$ is the Laplace transform of $u(\xi, n)$,

$$
\hat{u}(\xi, z)=\sum_{n=0}^{\infty} u(\xi, n) e^{-n z}, \quad \xi \in \mathbb{R}, z>0,
$$

then we see from (4.32) that

$$
\hat{u}(\xi, z)=1+\frac{e^{-z}}{\sqrt{2 \pi \sigma^{2}}} \int_{a}^{\infty} d \xi^{\prime} \hat{u}\left(\xi^{\prime}, z\right) \exp \left[-\left(\xi^{\prime}-\delta \xi\right)^{2} / 2 \sigma^{2}\right], \quad \xi \in \mathbb{R}, z>0
$$

It follows from (4.33) that for $\eta>0$,

$$
\sup _{\xi>a}\left[\hat{u}(\xi, z) e^{-\eta \xi}\right] \leq e^{-\eta a}+e^{-z} \sup _{\xi>a}\left[\hat{u}(\xi, z) e^{-\eta \xi}\right] \sup _{\xi>a} h_{\eta}(\xi),
$$

where $h_{\eta}(\xi)$ is given by the expression

$$
h_{\eta}(\xi)=\frac{1}{\sqrt{2 \pi \sigma^{2}}} \int_{a}^{\infty} d \xi^{\prime} \exp \left[\eta\left(\xi^{\prime}-\xi\right)-\left(\xi^{\prime}-\delta \xi\right)^{2} / 2 \sigma^{2}\right] .
$$


Evidently $\sup _{\xi>a} h_{\eta}(\xi)=1$ if $\eta=0$. We shall show that there is an $\eta>0$ such that $\sup _{\xi>a} h_{\eta}(\xi)<1$.

To see this we shall assume without loss of generality that $a<0$ and $0<\eta<1$. We choose $\alpha$ to satisfy $\delta<\alpha<1$, and for $\xi>0$ consider the integral

$$
\begin{aligned}
\int_{\alpha \xi}^{\infty} d \xi^{\prime} \exp \left[\eta\left(\xi^{\prime}-\xi\right)-\left(\xi^{\prime}-\delta \xi\right)^{2} / 2 \sigma^{2}\right] & \\
& =\sigma \exp [-\eta(1-\delta) \xi] \int_{K}^{\infty} \exp \left[\eta \sigma \zeta-\zeta^{2} / 2\right] d \zeta
\end{aligned}
$$

where $K=[\alpha-\delta] \xi / \sigma$. We have now that

$$
\begin{aligned}
\int_{K}^{\infty} \exp \left[\eta \sigma \zeta-\zeta^{2} / 2\right] d \zeta & =e^{\eta^{2} \sigma^{2} / 2} \int_{K-\eta \sigma}^{\infty} e^{-\zeta^{2} / 2} d \zeta \\
& \leq \exp \left[\eta^{2} \sigma^{2}+C(K-\eta \sigma) \eta \sigma\right] \int_{K}^{\infty} e^{-\zeta^{2} / 2} d \zeta
\end{aligned}
$$

where we have used (4.13) and assumed $K-\eta \sigma>1$. Taking $C>1$ and choosing $\alpha$ so that $(1-\delta)>C(\alpha-\delta)$, we conclude from the last two inequalities that there exists $\xi_{0}>0$ depending only on $\sigma$ and $\alpha$, such that

$$
\begin{aligned}
\int_{\alpha \xi}^{\infty} d \xi^{\prime} \exp [ & \left.\eta\left(\xi^{\prime}-\xi\right)-\left(\xi^{\prime}-\delta \xi\right)^{2} / 2 \sigma^{2}\right] \\
\leq & \exp [-\eta \xi\{(1-\delta)-C(\alpha-\delta)\}] \int_{\alpha \xi}^{\infty} d \xi^{\prime} \exp \left[-\left(\xi^{\prime}-\delta \xi\right)^{2} / 2 \sigma^{2}\right]
\end{aligned}
$$

provided $\xi>\xi_{0}$. It follows easily that

$$
h_{\eta}(\xi) \leq \exp [-\rho \eta \xi], \quad \xi>\xi_{0}, \quad 0<\eta<1,
$$

where $\rho=\min [(1-\delta)-C(\alpha-\delta), 1-\alpha]$. One can also see that we may choose $\eta>0$ sufficiently small such that $\sup _{a<\xi<\xi_{0}} h_{\eta}(\xi)<1$. Combining this with (4.35), we conclude that $\sup _{\xi>a} h_{\eta}(\xi)<1$ for sufficiently small $\eta>0$. Now, on letting $z \rightarrow 0$ in (4.34), we see that

$$
\sum_{n=1}^{\infty} P\left(\xi_{m}>a, 1 \leq m \leq n \mid \xi_{0}=\xi\right)<\infty .
$$

Hence, by the Borel-Cantelli lemma, $\liminf \operatorname{in}_{n \rightarrow \infty} \xi_{n} \leq a$ with probability 1. Now (4.30) implies that $\liminf _{n \rightarrow \infty} Z_{\varepsilon}\left(s_{n}\right) / \sqrt{T-s_{n}}=-\infty$ with probability 1.

Theorem 4.4. Let $\lambda_{\varepsilon}(\cdot, \cdot)$ be the optimal controller defined by (3.56). Then the $S D E(1.14)$ has a unique strong solution $y_{\varepsilon}(s)$ for $t \leq s<T$, with initial condition $y_{\varepsilon}(t)=y$. Furthermore $\liminf _{s \rightarrow T} y_{\varepsilon}(s)>x$ with probability 1 .

Proof. To show the existence and uniqueness of a solution to (1.14) we argue as in Lemma 3.5. Thus for $y_{0}<y$ let $\tau\left(y_{0}\right)=\inf \left\{s \geq t: s<T, y_{\varepsilon}(s)=y_{0}\right\}$. Since $\lambda_{\varepsilon}\left(y^{\prime}, s\right) \geq b\left(y^{\prime}, s\right)$ for $y^{\prime} \in \mathbb{R}$ and $s<T$, it follows that $\lim _{y_{0} \rightarrow-\infty} P\left(\tau\left(y_{0}\right)<T\right)=0$. 
Hence by the Lipschitz property of $\lambda_{\varepsilon}\left(y^{\prime}, s\right)$ for $y^{\prime} \geq y_{0}$ and $t \leq s \leq T-\delta$, we obtain for any $\delta>0$ a unique strong solution to (1.14) up to time $T-\delta$. Letting $\delta \rightarrow 0$ we get existence and uniqueness in the interval $t \leq s<T$.

To show that $\liminf _{s \rightarrow T} y_{\varepsilon}(s)>x$ we consider for $y_{0}<y$ and $t \leq s<T$, solutions $y_{\varepsilon}(s)$ of (1.14) with $y_{\varepsilon}(t)=y$ such that $\tau\left(y_{0}\right)=T$. From (4.2) and the fact that $b(\cdot, s)$ is uniformly Lipschitz for $t \leq s \leq T$, we see that there exists $s_{0}$ with $t \leq s_{0}<T$, and $\mu_{0}>0$, such that such that

$$
d y_{\varepsilon}(s) \geq\left(b(x, s)+\frac{\mu_{0}\left[x-y_{\varepsilon}(s)\right]}{T-s}\right) d s+\sqrt{\varepsilon} d W(s), \quad s_{0} \leq s<T,
$$

on paths $y_{\varepsilon}(\cdot)$ for which $\tau\left(y_{0}\right)=T$. It follows then from (4.36) and Lemma 4.3 that on paths $y_{\varepsilon}(\cdot)$ for which $\tau\left(y_{0}\right)=T$ one has in fact $\liminf _{s \rightarrow T} y_{\varepsilon}(s) \geq x$ with probability 1 . Letting $y_{0} \rightarrow-\infty$, we conclude that $\liminf _{s \rightarrow T} y_{\varepsilon}(s) \geq x$ with probability 1 on all paths $y_{\varepsilon}(\cdot)$ for which $y_{\varepsilon}(t)=y$.

Next, for $\eta>0$ and $s_{0}<T$, let $U_{\eta, s_{0}}=\left\{y_{\varepsilon}(\cdot): y_{\varepsilon}(t)=y, y_{\varepsilon}(s) \geq x-\eta, s_{0} \leq\right.$ $s<T\}$. If $\eta$ and $T-s_{0}$ are sufficiently small it follows from (4.2) that we can take $\mu_{0}>1 / 2$ for a path $y_{\varepsilon}(\cdot) \in U_{\eta, s_{0}}$. Hence by Lemma 4.3 we have that $\limsup _{s \rightarrow T}\left[y_{\varepsilon}(s)-x\right] / \sqrt{T-s}=+\infty$ with probability 1 for all paths $y_{\varepsilon}(\cdot) \in U_{\eta, s_{0}}$. Since $\lim _{s_{0} \rightarrow T} P\left(U_{\eta, s_{0}}\right)=1$, we conclude that $\limsup _{s \rightarrow T}\left[y_{\varepsilon}(s)-x\right] / \sqrt{T-s}=$ $+\infty$ with probability 1 on all solutions to (1.14) with $y_{\varepsilon}(t)=y$.

For $K>0$ we define a stopping time $\tau_{K}$ by

$$
\tau_{K}=\inf \left\{s \geq t: s<T, y_{\varepsilon}(s)-x=K \sqrt{T-s}\right\} .
$$

We have just shown that $P\left(\tau_{K}<T\right)=1$. Consider now a solution $y_{\varepsilon}(s)$ to $(1.14)$ for $s_{1} \leq s<T$, with initial condition $y_{\varepsilon}\left(s_{1}\right)=y_{1}$. Now $y_{\varepsilon}(s) \geq Y_{\varepsilon}(s)$ for $s_{1} \leq s<T$, where $Y_{\varepsilon}(s)$ is the solution to (1.5) with $Y_{\varepsilon}\left(s_{1}\right)=y_{1}$. From $(3.4)$ we conclude that

$$
\inf _{s_{1} \leq s<T} y_{\varepsilon}(s) \geq y_{1}-C \sup _{s_{1} \leq s<T}\left|\int_{s_{1}}^{s} b\left(y_{1}, s^{\prime}\right) d s^{\prime}+\sqrt{\varepsilon}\left[W(s)-W\left(s_{1}\right)\right]\right|,
$$

for some constant $C$. We take now $s_{1} \geq t$ and $y_{1}=x+K \sqrt{T-s_{1}}$ in (4.37). It is clear that there is a constant $K_{0}>0$ such that, for $K>K_{0}$,

$$
\begin{aligned}
P\left(\inf _{s_{1} \leq s<T} y_{\varepsilon}(s) \leq x\right) & \leq P\left(\sqrt{\varepsilon} \sup _{s_{1} \leq s<T}\left|W(s)-W\left(s_{1}\right)\right|>K \sqrt{T-s_{1}} / 2\right) \\
& \leq 4 \varepsilon / K^{2}
\end{aligned}
$$

Taking $s_{1}=\tau_{K}$ in (4.38) we conclude that, for $K>K_{0}$, one has

$$
P\left(\liminf _{s \rightarrow T} y_{\varepsilon}(s) \leq x\right) \leq 4 \varepsilon / K^{2}
$$

Letting $K \rightarrow \infty$ yields the result. 
Corollary 4.5. Let $\lambda_{\varepsilon}(\cdot, \cdot)$ be the optimal controller defined by (3.56), and let $y_{\varepsilon}(s)$ be the corresponding solution to (1.14) with initial condition $y_{\varepsilon}(t)=y$. Then one has

$$
\lim _{\delta \rightarrow 0} q_{\varepsilon}\left(x, y_{\varepsilon}(T-\delta), T-\delta\right)=0 \text { with probability } 1
$$

Proof. We use the second inequality of (4.1) to obtain an estimate on $q_{\varepsilon}(x, y, T-\delta)$ when $y>x$. Since $q_{\varepsilon}(x, \cdot, T-\delta)$ is a positive decreasing function we have that

$$
\begin{aligned}
& q_{\varepsilon}(x, y, T-\delta) \leq C \varepsilon \exp \left[-\frac{(x-y)^{2}}{2 \varepsilon \delta}\right]+C_{1}(\varepsilon \delta)^{1 / 4}, \quad x<y<x+(\varepsilon \delta)^{1 / 4} \\
& q_{\varepsilon}(x, y, T-\delta) \leq C_{1}(\varepsilon \delta)^{1 / 4}, \quad y>x+(\varepsilon \delta)^{1 / 4}
\end{aligned}
$$

for some constants $C$ and $C_{1}$. Now (4.39) follows from (4.40) and Theorem 4.4.

\section{Proof of Theorem 1.2}

The problem of estimating $\partial q_{\varepsilon}(x, y, t) / \partial y$ is closely related to the problem of estimating certain conditional probabilities. For $0<\delta<T / 2$ we shall consider the conditional probability $P\left(Y_{\varepsilon}(T-\delta) \in U \mid Y_{\varepsilon}(0)=y, Y_{\varepsilon}(T)=0\right)$, where $Y_{\varepsilon}(s)$, defined for $0 \leq s \leq T$, satisfies the $\operatorname{SDE}(1.5)$ and $U$ is an arbitrary open set. In the linear approximation $b(y, s)=A(s) y$ the variable $Y_{\varepsilon}(T)$ conditioned on $Y_{\varepsilon}(0)=y$ is Gaussian with mean $\Lambda(T) y$ and variance $\varepsilon \sigma^{2}(T)$, where $\Lambda(T)$ and $\sigma^{2}(T)$ are given by the formulas

$$
\Lambda(T)=\exp \left[\int_{0}^{T} A(s) d s\right], \quad \sigma^{2}(T)=\int_{0}^{T} \exp \left[2 \int_{s}^{T} A\left(s^{\prime}\right) d s^{\prime}\right] d s .
$$

The variable $Y_{\varepsilon}(T-\delta)$ conditioned on $Y_{\varepsilon}(0)=y, Y_{\varepsilon}(T)=0$, is also Gaussian with mean and variance given by the formulas

$$
\begin{aligned}
E\left[Y_{\varepsilon}(T-\delta) \mid Y_{\varepsilon}(0)\right. & \left.=y, Y_{\varepsilon}(T)=0\right]=\frac{\Lambda(T-\delta)}{\sigma^{2}(T)} y \int_{T-\delta}^{T} \exp \left[2 \int_{s}^{T} A\left(s^{\prime}\right) d s^{\prime}\right] d s, \\
\operatorname{Var}\left[Y_{\varepsilon}(T-\delta) \mid Y_{\varepsilon}(0)\right. & \left.=y, Y_{\varepsilon}(T)=0\right]=\frac{\varepsilon \sigma^{2}(T-\delta)}{\sigma^{2}(T)} \int_{T-\delta}^{T} \exp \left[2 \int_{s}^{T} A\left(s^{\prime}\right) d s^{\prime}\right] d s .
\end{aligned}
$$

The mean in $(5.2)$ is equal to $y_{\min }(T-\delta)$, where $y_{\min }(s)$, defined for $0 \leq$ $s \leq T$, is the unique minimizer for the functional $\mathcal{F}[y(\cdot)]$ of $(2.8)$ conditioned on $y(0)=y$ and $y(T)=0$. One sees easily from (5.2) that there are positive universal constants $C_{1}$ and $C_{2}$ such that

$$
\begin{aligned}
& \frac{C_{2} \delta y}{T} \leq E\left[Y_{\varepsilon}(T-\delta) \mid Y_{\varepsilon}(0)=y, Y_{\varepsilon}(T)=0\right] \leq \frac{C_{1} \delta y}{T}, \\
& C_{1} \varepsilon \delta \leq \operatorname{Var}\left[Y_{\varepsilon}(T-\delta) \mid Y_{\varepsilon}(0)=y, Y_{\varepsilon}(T)=0\right] \leq C_{2} \varepsilon \delta
\end{aligned}
$$


for $y<0$ provided $0<\delta<T / 2$ and $A T<1$. It follows from (5.3) that there are positive universal constants $C_{3}, \gamma_{3}, C_{4}$, and $\gamma_{4}$ such that

$$
\begin{aligned}
& P\left(Y_{\varepsilon}(T-\delta)<\frac{C_{3} \delta y}{T} \mid Y_{\varepsilon}(0)=y, Y_{\varepsilon}(T)=0\right) \leq \exp \left[-\frac{\gamma_{3} \delta y^{2}}{\varepsilon T^{2}}\right], \quad y<-T \sqrt{\varepsilon / \delta}, \\
& P\left(Y_{\varepsilon}(T-\delta)>\frac{C_{4} \delta y}{T} \mid Y_{\varepsilon}(0)=y, Y_{\varepsilon}(T)=0\right) \leq \exp \left[-\frac{\gamma_{4} \delta y^{2}}{\varepsilon T^{2}}\right], \quad y<-T \sqrt{\varepsilon / \delta},
\end{aligned}
$$

provided $0<\delta<T / 2$ and $A T<1$.

Evidently (5.4) proves Theorem 1.2 in the case of $b(y, \cdot)$ linear in $y \in \mathbb{R}$. Therefore we need to show that $(5.4)$ continues to hold for nonlinear $b(\cdot, \cdot)$ satisfying $(1.1)$ and $b(0, \cdot) \equiv 0$. Towards this goal we first observe that in the linear case there are positive universal constants $C_{3}, \gamma_{3}, C_{4}$, and $\gamma_{4}$ such that if $\mathcal{F}_{\min }=\mathcal{F}\left[y_{\min }(\cdot)\right]$ then

$$
\begin{array}{ll}
\mathcal{F}[y(\cdot)]-\mathcal{F}_{\min } \geq \gamma_{3} \delta y^{2} / T^{2} \quad \text { if } \quad & y(T-\delta)<C_{3} \delta y / T, \\
\mathcal{F}[y(\cdot)]-\mathcal{F}_{\min } \geq \gamma_{4} \delta y^{2} / T^{2} \quad \text { if } \quad & y(T-\delta)>C_{4} \delta y / T,
\end{array}
$$

provided $0<\delta<T / 2, A T<1$, and $y<0$. For nonlinear $b(\cdot, \cdot)$ there is not necessarily a unique minimizer of the functional $\mathcal{F}[y(\cdot)]$ subject to $y(0)=y<0$ and $y(T)=0$. Nevertheless, if $\mathcal{F}_{\text {min }}$ denotes now the minimum of $\mathcal{F}[y(\cdot)]$ then $(5.5)$ continues to hold.

Lemma 5.1. Let $b(\cdot, \cdot)$ satisfy $(1.1)$ and $b(0, \cdot) \equiv 0$. Assume further that $y<0$, $\delta<T / 2$, and $A T<1$, and that $\mathcal{F}_{\min }$ is the minimum of the functional $\mathcal{F}[y(\cdot)]$ of (2.8) subject to $y(0)=y$ and $y(T)=0$. Then (5.5) holds for some positive universal constants $C_{3}, \gamma_{3}, C_{4}$, and $\gamma_{4}$, on any path $y(s)$, defined for $0 \leq s \leq T$, satisfying $y(0)=y$ and $y(T)=0$.

Proof. We first show that there are positive universal constants $C_{1}$ and $C_{2}$ such that

$$
C_{1} y^{2} / T \leq \mathcal{F}_{\min } \leq C_{2} y^{2} / T
$$

The upper bound in (5.6) can be obtained by estimating $\mathcal{F}[y(\cdot)]$ for the linear path $y(s)=(T-s) y / T$, defined for $0 \leq s \leq T$. To get the lower bound we consider a path $y(s)$, defined for $0 \leq s \leq T$, satisfying $y(0)=y$ and $y(T)=0$, and write

$$
\frac{d y}{d s}=b(y(s), s)+f(s)=A(s) y(s)+f(s),
$$

where $|A(s)| \leq A$ for $0 \leq s \leq T$. Evidently we see from (5.7) that

$$
y=y(0)=-\int_{0}^{T} \exp \left[-\int_{0}^{s} A\left(s^{\prime}\right) d s^{\prime}\right] f(s) d s .
$$

Since $A T<1$ we conclude that

$$
|y| \leq e \int_{0}^{T}|f(s)| d s \leq e \sqrt{T}\left[\int_{0}^{T}|f(s)|^{2} d s\right]^{1 / 2},
$$

whence we obtain the lower bound in (5.6) with $C_{1}=1 / 2 e^{2}$. 
To prove the first inequality in (5.5) we consider for $\lambda>1$ a path $y_{\lambda}(s)$, defined for $0 \leq s \leq T$, satisfying $y_{\lambda}(0)=y, y_{\lambda}(T)=0$, and $y_{\lambda}(T-\delta)=\lambda \delta y / T$. We derive a second path $y_{\lambda}^{*}$ from $y_{\lambda}$ by setting $y_{\lambda}^{*}(s)=0$ for $T-\delta<s<T$, and $y_{\lambda}^{*}(s)=y_{\lambda}(s)-s \lambda \delta y / T(T-\delta)$ for $0<s<T-\delta$. Thus $y_{\lambda}^{*}(\cdot)$ is continuous and $y_{\lambda}^{*}(0)=y$ and $y_{\lambda}^{*}(T)=0$, whence we must have $\mathcal{F}\left[y_{\lambda}^{*}(\cdot)\right] \geq \mathcal{F}_{\min }$. We also have that

$$
\begin{aligned}
\mathcal{F}\left[y_{\lambda}(\cdot)\right]= & \frac{1}{2} \int_{0}^{T-\delta}\left[\frac{d y_{\lambda}^{*}(s)}{d s}+\frac{\lambda \delta y}{T(T-\delta)}-b\left(y_{\lambda}^{*}(s)+s \lambda \delta y / T(T-\delta), s\right)\right]^{2} d s \\
& +\frac{1}{2} \int_{T-\delta}^{T}\left[\frac{d y_{\lambda}}{d s}-b\left(y_{\lambda}(s), s\right)\right]^{2} d s
\end{aligned}
$$

Arguing as we did to get the lower bound in (5.6) we see that

$$
\frac{1}{2} \int_{T-\delta}^{T}\left[\frac{d y_{\lambda}(s)}{d s}-b\left(y_{\lambda}(s), s\right)\right]^{2} d s \geq \frac{\lambda^{2} \delta y^{2}}{2 e^{2} T^{2}}
$$

The first term on the right-hand side of (5.8) is bounded below by

$$
\mathcal{F}\left[y_{\lambda}^{*}(\cdot)\right]-\frac{2 \lambda \delta|y|}{T(T-\delta)} \int_{0}^{T-\delta}\left|\frac{d y_{\lambda}^{*}(s)}{d s}-b\left(y_{\lambda}^{*}(s), s\right)\right| d s,
$$

where we have used the fact that $A T<1$. It follows then from $(5.9)$ and $(5.10)$ that

$$
\mathcal{F}\left[y_{\lambda}(\cdot)\right] \geq \mathcal{F}\left[y_{\lambda}^{*}(\cdot)\right]-\frac{2 \sqrt{2} \lambda \delta|y|}{T \sqrt{T-\delta}} \mathcal{F}\left[y_{\lambda}^{*}(\cdot)\right]^{1 / 2}+\frac{\lambda^{2} \delta y^{2}}{2 e^{2} T^{2}}
$$

Observe now from (5.6), (5.8), and (5.9) that there is a universal constant $C_{3}$ such that if $\lambda \delta / T>C_{3}$ then $\mathcal{F}\left[y_{\lambda}(\cdot)\right]-\mathcal{F}_{\text {min }} \geq \lambda^{2} \delta y^{2} / 2 e^{2} T^{2}$. Suppose now that $\lambda \delta / T<C_{3}$. If $\mathcal{F}\left[y_{\lambda}^{*}(\cdot)\right] \geq\left[2 C_{2}+64 C_{3}^{2}\right] y^{2} / T$ it follows from (5.11) that $\mathcal{F}\left[y_{\lambda}(\cdot)\right]-\mathcal{F}_{\min } \geq \lambda^{2} \delta y^{2} / 2 e^{2} T^{2}$. On the other hand if $\mathcal{F}\left[y_{\lambda}^{*}(\cdot)\right] \leq\left[2 C_{2}+64 C_{3}^{2}\right] y^{2} / T$ we see again from (5.11) that $\mathcal{F}\left[y_{\lambda}(\cdot)\right]-\mathcal{F}_{\min } \geq \lambda^{2} \delta y^{2} / 4 e^{2} T^{2}$ if $\lambda>\lambda_{0} \geq 1$ for some universal $\lambda_{0}$. We have proven the first inequality of (5.5).

We turn to the proof of the second inequality in $(5.5)$. Let $y_{1}(\cdot)$ be a trajectory satisfying $y_{1}(0)=y$ and $y_{1}(T)=0$, and set $\tau=\inf \left\{s \geq 0: y_{1}(s)=0\right\}$. Suppose now that $\tau \leq T-\delta$. From (5.6) one has that $\mathcal{F}\left[y_{1}(\cdot)\right] \geq C_{1} y^{2} / \tau$, and so the second inequality of (5.5) follows if $\tau<C_{1} T / 2 C_{2}$. We assume therefore that $C_{1} T / 2 C_{2}<$ $\tau \leq T-\delta$. Let $y_{\min }(\cdot)$ be a minimizing path for the functional $\mathcal{F}[y(\cdot)]$ subject to the conditions $y(0)=y$ and $y(s)=0$ for $\tau \leq s \leq T$. Then $\mathcal{F}\left[y_{1}(\cdot)\right] \geq \mathcal{F}\left[y_{\min }(\cdot)\right]$. From (2.14) we see that there are positive universal constants $C_{3}$ and $C_{4}$ such that

$$
\frac{C_{3}|y|}{T} \leq \frac{d y_{\min }(s)}{d s}-b\left(y_{\min }(s), s\right) \leq \frac{C_{4}|y|}{T}, \quad 0 \leq s \leq \tau .
$$

Since $A T<1$ we conclude from (5.12) that

$$
e C_{4}(\tau-s) y / T \leq y_{\min }(s) \leq C_{3}(\tau-s) y / e T, \quad 0 \leq s \leq \tau
$$


It is clear that there is a positive universal constant $\varepsilon_{0}$ such that for $0<\varepsilon<\varepsilon_{0}$ we may define a path $y_{\varepsilon}(\cdot)$ as follows: $y_{\varepsilon}(s)=y_{\min }(s)$ for $0 \leq s \leq \tau-\varepsilon \delta$, and $y_{\varepsilon}(s)=(T-s) y_{\min }(\tau-\varepsilon \delta) /(T-\tau+\varepsilon \delta)$ for $\tau-\varepsilon \delta \leq s \leq T$. Since $y_{\varepsilon}(\cdot)$ is continuous, $y_{\varepsilon}(0)=y$ and $y_{\varepsilon}(T)=0$, we have that $\mathcal{F}\left[y_{\varepsilon}(\cdot)\right] \geq \mathcal{F}_{\min }$. From $(5.12)$ and (5.13) we also have that

$$
\mathcal{F}\left[y_{\min }(\cdot)\right]-\mathcal{F}\left[y_{\varepsilon}(\cdot)\right] \geq \varepsilon \delta C_{3}^{2} y^{2} / 2 T^{2}-e^{2} C_{4}^{2} \varepsilon^{2} \delta y^{2} / 2(1+\varepsilon) T^{2},
$$

where we have used the fact that $\tau \leq T-\delta$. Evidently the second inequality of (5.5) follows from (5.14) by choosing $\varepsilon=\min \left[1, C_{3} / 2 e C_{4}\right]^{2}$.

To complete the proof of the second inequality of (5.5) we need to consider the case $T-\delta \leq \tau \leq T$. It is evident that if $C \delta y / T<y_{1}(T-\delta) \leq 0$ for sufficiently small universal $C>0$ we may repeat the argument of the previous paragraph. Hence the result follows in all cases.

We begin the proof of (5.4) by sharpening the estimate (4.27) on the Green's function $G(y, x, t, T)$ defined by (1.4).

Lemma 5.2. Suppose $b(\cdot, \cdot)$ satisfies $(1.1)$ and, in addition, $b(0, \cdot) \equiv 0$. Then there are universal positive constants $C$ and $\delta$ such that the Green's function $G$ defined by (1.4) satisfies the inequalities

$$
\begin{aligned}
& G(y, 0,0, T) \leq \frac{1}{\sqrt{2 \pi \varepsilon T}} \exp \left[\frac{-y^{2}}{2 \varepsilon T(1+C A T)}+C A T\right], \\
& G(y, 0,0, T) \geq \frac{1}{\sqrt{2 \pi \varepsilon T}} \exp \left[\frac{-y^{2}(1+C A T)}{2 \varepsilon T}-C A T\right],
\end{aligned}
$$

provided $A T \leq \delta$.

Proof. We shall first prove (5.16). Suppose that we have shown that

$$
G(y, 0, t, T) \geq \frac{1}{\sqrt{2 \pi \varepsilon(T-t)}} \exp \left[\frac{-y^{2}}{2 \varepsilon(T-t)}\{1+C A(T-t)\}-C A(T-t)\right]
$$

for $T-t=T / 2^{N}$, where $N$ is some integer $N \geq 1$. We shall show that for a sufficiently large universal constant $C>0,(5.17)$ also holds for $T-t=T / 2^{N-1}$. The inequality (5.16) will then follow by induction if we can prove (5.17) holds as $T-t \rightarrow 0$.

Defining $t_{N}$ by $T-t_{N}=T / 2^{N}, N \in \mathbb{N}$, we see in a similar way to how we derived (4.23) that

$$
\begin{aligned}
& G\left(y, 0, t_{N-1}, T\right)^{1-\alpha} \\
& \quad \geq \int_{-\infty}^{\infty} \frac{1}{\sqrt{2 \pi \varepsilon T / 2^{N}}} \exp \left[-\frac{(y-z)^{2}}{2 \varepsilon T / 2^{N}}\right] F_{N}(y, z) G\left(z, 0, t_{N}, T\right)^{1-\alpha} d z
\end{aligned}
$$

where $F_{N}(y, z)$ is given by the formula,

$$
F_{N}(y, z)=\exp \left\{-\frac{A^{2}(1-\alpha)}{6 \alpha \varepsilon} \frac{T}{2^{N}}\left(y^{2}+z y+z^{2}\right)-\frac{A^{2}(1-\alpha)}{12 \alpha} \frac{T^{2}}{2^{2 N}}\right\} .
$$


Assuming now that we may bound $G\left(z, 0, t_{N}, T\right)$ according to $(5.17)$, then the right-hand side of (5.18) becomes a Gaussian integral which we can evaluate. Taking $\alpha=A T / 2^{N}$ in (5.18) and $C_{N}$ to be the constant $C$ in (5.17) when $t=t_{N}$, we see that it is possible to take $C_{N-1}=5 C_{N} / 8+2$ provided $N \geq 1$ and $\delta \leq 1$. We conclude therefore that

$$
C_{0}=\frac{16}{3}\left[1-\left(\frac{5}{8}\right)^{N}\right]+\left(\frac{5}{8}\right)^{N} C_{N}, \quad N \geq 1
$$

The inequality (5.16) follows from (5.20) if we can show that

$$
\lim _{N \rightarrow \infty} 5^{N} C_{N} / 8^{N}=0 .
$$

We can do this by the same method we used to derive (4.27).

We shall show that the inequality (5.16) holds with a constant $C=C(A T)$ which can diverge as $T \rightarrow 0$, but in a mild, in fact logarithmic, way. As in (4.23) we write

$$
G(y, 0,0, T)^{1-\alpha}
$$

$$
\geq \int_{-\eta}^{\eta} \frac{1}{\sqrt{2 \pi \varepsilon(T-\Delta)}} \exp \left[-\frac{(y-z)^{2}}{2 \varepsilon(T-\Delta)}\right] F_{0}(y, z) G_{D, \eta}(z, 0, T-\Delta, T)^{1-\alpha} d z,
$$

where $G_{D, \eta}$ is the Dirichlet Green's function for the equation (1.2) on the interval $[-\eta, \eta]$. The function $F_{0}$ is given by the formula (5.19) when $N=0$, and we take $\alpha=A T$. As in Lemma 3.4 we use perturbation theory to estimate $G_{D, \eta}$. In order for the perturbation expansion to converge we need that

$$
\eta=K \sqrt{\varepsilon \Delta}, \quad(A \eta)^{2} \Delta=\nu \varepsilon,
$$

where $K>1$ and $\nu<<1$. In this case the lower bound

$$
\begin{aligned}
G_{D, \eta}(z, 0, T-\Delta, T) \geq & \frac{1}{\sqrt{2 \pi \varepsilon \Delta}}\left[\exp \left\{\frac{-z^{2}}{2 \varepsilon \Delta}\right\}-C_{1} e^{-K^{2} / 4}\right. \\
& \left.-C_{2}(\rho) \nu^{1 / 2} \exp \left\{\frac{-z^{2}}{2 \varepsilon(1+\rho) \Delta}\right\}\right], \quad|z|<\eta
\end{aligned}
$$

holds, where $C_{1}$ is a universal constant, $\rho>0$ can be arbitrary, and $C_{2}(\rho)$ is a constant depending only on $\rho$. We shall substitute the right-hand side of (5.23) into (5.21), choosing $\Delta / T, K$, and $\nu$ to be powers of $A T$, in order to obtain a lower bound as in (5.16).

Consider the situation when we approximate $G_{D, \eta}$ by the first term on the right-hand side of (5.23). From (5.21) we have that

$$
\begin{aligned}
G(y, 0,0, T)^{1-\alpha} \geq \inf _{|z|<\eta}\left\{\exp \left[-\frac{(y-z)^{2}}{2 \varepsilon(T-\Delta)}\right] F_{0}(y, z)\right\} \\
\cdot \frac{1}{\sqrt{2 \pi \varepsilon(T-\Delta)}} \int_{-\eta}^{\eta} G_{D, \eta}(z, 0, T-\Delta, T)^{1-\alpha} d z .
\end{aligned}
$$


Observe now that

$$
\begin{aligned}
& \left\{\frac{1}{\sqrt{2 \pi \varepsilon(T-\Delta)}} \int_{-\eta}^{\eta} \frac{1}{(2 \pi \varepsilon \Delta)^{(1-\alpha) / 2}} \exp \left[-\frac{z^{2}(1-\alpha)}{2 \varepsilon \Delta}\right] d z\right\}^{1 /(1-\alpha)} \\
& \geq \frac{1}{\sqrt{2 \pi \varepsilon T}}\left[1-e^{-K^{2} / 4}\right] \exp \left[-C k_{0} A T|\log (A T)|\right]
\end{aligned}
$$

for some universal constant $C$, provided we choose $\Delta / T=(A T)^{k_{0}}$ with $k_{0}>1$ and $A T \leq 1 / 2$. From (5.25) it is clear that it is sufficient to choose $K=(A T)^{-k_{1}}$ for any $k_{1}>0$, whence (5.22) implies that $\nu^{1 / 2}=(A T)^{k_{0}+1-k_{1}}$. If we now use the inequality

$$
2 \eta|y| / \varepsilon T \leq(A T)^{k_{0} / 2-k_{1}}\left[y^{2} / \varepsilon T+1\right],
$$

and choose $k_{0}>2 k_{1}+2$, we conclude from (5.24) and (5.25) that (5.16) holds with $C=C^{\prime}|\log (A T)|$ for some universal constant $C^{\prime}$. We may easily extend this argument to apply to the actual lower bound (5.23) on $G_{D, \eta}$ by using the inequality

$$
\max [a-b, 0]^{1-\alpha} \geq(a-b), \quad a, b>0, a<1 .
$$

Returning now to (5.20), it follows that we may take $C_{N}=O(N)$, and so we conclude that $\lim _{N \rightarrow 0} 5^{N} C_{N} / 8^{N}=0$. We have therefore shown that (5.16) holds for some universal constant $C>0$ provided $A T<\delta$ where $\delta$ is also universal.

To prove (5.15) we use a method similar to that used in the proof of the lower bound. Suppose we have shown that

$$
G(y, 0, t, T) \leq \frac{1}{\sqrt{2 \pi \varepsilon(T-t)}} \exp \left[\frac{-y^{2}}{2 \varepsilon(T-t)[1+C A(T-t)]}+C A(T-t)\right]
$$

for $T-t=T / 2^{N}$ where $N$ is some integer $N \geq 1$. We shall show that for a sufficiently large universal constant $C>0$, the inequality (5.27) also holds for $T-t=T / 2^{N-1}$. Analogously to (5.18) the inequality

$$
\begin{aligned}
& G\left(y, 0, t_{N-1}, T\right)^{1+\alpha} \\
& \quad \leq \int_{-\infty}^{\infty} \frac{1}{\sqrt{2 \pi \varepsilon T / 2^{N}}} \exp \left[-\frac{(y-z)^{2}}{2 \varepsilon T / 2^{N}}\right] F_{N}(y, z) G\left(z, 0, t_{N}, T\right)^{1+\alpha} d z
\end{aligned}
$$

holds, where $F_{N}(y, z)$ is given by $(4.20)$ with $g \equiv 0, x=0$, and $t=t_{N-1}$, and $T$ is replaced by $t_{N}$. Using the fact that one knows the probability density function of $\sup _{t \leq s \leq T} W(s-t)$ we see that $F_{N}(y, z)$ is bounded above by

$$
F_{N}(y, z) \leq \exp \left\{\frac{A^{2}(1+\alpha)}{3 \alpha \varepsilon} \frac{T}{2^{N}}\left(y^{2}+z y+z^{2}\right)+\frac{K_{0} A^{2}(1+\alpha)}{\alpha} \frac{T^{2}}{2^{2 N}}\right\}
$$

for a universal constant $K_{0}>0$, where we are assuming $\alpha=A T / 2^{N}<\delta$ and $\delta$ is a sufficiently small universal constant. Letting $C_{N}$ be the constant $C$ in $(5.27)$ when $t=t_{N}$, we see from (5.28) and (5.29) that it is possible to take $C_{N-1}=$ 
$2 C_{N} / 3+K_{0}+4, N \geq 1$, provided $A T<\delta$ and $\delta$ is sufficiently small. Arguing as before, in order to complete the proof of (5.15) we need to show that

$$
\lim _{N \rightarrow \infty} 2^{N} C_{N} / 3^{N}=0 .
$$

To do this we show that (5.15) holds with a constant $C=C(A T)$ which can diverge as $T \rightarrow 0$, although only in a logarithmic way. We use the inequality

$$
\begin{aligned}
& G(y, 0,0, T)^{1+\alpha} \\
& \leq \int_{-\infty}^{\infty} \frac{1}{\sqrt{2 \pi \varepsilon(T-\Delta)}} \exp \left[-\frac{(y-z)^{2}}{2 \varepsilon(T-\Delta)}\right] F_{0}(y, z) G(z, 0, T-\Delta, T)^{1+\alpha} d z,
\end{aligned}
$$

where $F_{0}$ is given by the right-hand side of (5.29) when $N=0$.

Choosing $\eta$ and $\nu$ as in (5.22) we see by perturbation theory that there is an upper bound

$$
\begin{aligned}
& G_{D, \eta}(z, 0, T-\Delta, T) \\
& \quad \leq \frac{1}{\sqrt{2 \pi \varepsilon \Delta}}\left[\exp \left\{-\frac{z^{2}}{2 \varepsilon \Delta}\right\}+C_{2}(\rho) \nu^{1 / 2} \exp \left\{-\frac{z^{2}}{2 \varepsilon(1+\rho) \Delta}\right\}\right],
\end{aligned}
$$

for $|z|<\eta$, analogous to the lower bound (5.23). Suppose now that $0<z<\eta / 2$. Then

$$
\begin{aligned}
& G(z, 0, T-\Delta, T) \\
& \quad=G_{D, \eta}(z, 0, T-\Delta, T)+\int_{T-\Delta}^{T} d t \sum_{\sigma= \pm 1} \rho(\sigma, t) G_{D, \eta}(\sigma \eta / 2,0, t, T),
\end{aligned}
$$

where $\rho(\sigma, t)$ is the density of the hitting time at $\sigma \eta / 2$ for paths of the diffusion $Y_{\varepsilon}(\cdot)$ satisfying $(1.5)$ with $Y_{\varepsilon}(T-\Delta)=z$, which exit the interval $[-\eta, \eta]$ before time $T$ and make their final returns through $\sigma \eta$. Since $|z|<\eta / 2$, it is evident that

$$
\begin{aligned}
\int_{T-\Delta}^{T} \sum_{\sigma= \pm 1} \rho(\sigma, t) d t & \leq 1-\int_{-\eta}^{\eta} G_{D, \eta}\left(z, z^{\prime}, T-\Delta, T\right) d z^{\prime} \\
& \leq C_{1} e^{-K^{2} / 16}+C_{2} \nu^{1 / 2}
\end{aligned}
$$

for universal constants $C_{1}$ and $C_{2}$. One can also see from (5.31) on replacing $T-\Delta$ by $t>T-\Delta$ that

$$
G_{D, \eta}(\eta / 2,0, t, T) \leq \frac{C_{3}}{\sqrt{2 \pi \varepsilon \Delta}} e^{-K^{2} / 16}, \quad T-\Delta<t<T,
$$

for some universal constant $C_{3}$.

Substituting the right-hand sides of (5.33) and (5.34) into the right-hand side of (5.32), we conclude from (5.31) that

$$
\begin{aligned}
G(z, 0, T-\Delta, T) \leq & \frac{1}{\sqrt{2 \pi \varepsilon \Delta}}\left[\exp \left\{-\frac{z^{2}}{2 \varepsilon \Delta}\right\}+C_{4} e^{-K^{2} / 16}\right. \\
& \left.+C_{2}(\rho) \nu^{1 / 2} \exp \left\{-\frac{z^{2}}{2 \varepsilon(1+\rho) \Delta}\right\}\right], \quad|z|<\eta / 2 .
\end{aligned}
$$


We may estimate $G(z, 0, T-\Delta, T)$ similarly for $|z|>\eta / 2$. Thus we have

$$
G(z, 0, T-\Delta, T)=\int_{T-\Delta}^{T} d t \rho(t) G(\eta / 2,0, t, T), \quad z>\eta / 2,
$$

where again $\rho(\cdot)$ is the hitting time density at $\eta / 2$. Evidently we have that

$$
\int_{T-\Delta}^{T} \rho(t) d t=P\left(\inf _{T-\Delta<t<T} Y_{\varepsilon}(t)<\eta / 2 \mid Y_{\varepsilon}(T-\Delta)=z\right) .
$$

It is easy to bound the right-hand side of (5.37) by using the inequality $b(y, s) \geq$ $-A y$ for $y>0$ in (1.5), and estimating the probability on the right-hand side of (5.37) for the corresponding Gaussian process. Assuming that $A \Delta<1 / 10$ and $z>2 \eta$ we have that

$$
\int_{T-\Delta}^{T} \rho(t) d t \leq P\left(\inf _{0<t<\Delta} \int_{0}^{t} e^{A s} d W(s)<-z / 2 \sqrt{\varepsilon}\right),
$$

where $W(\cdot)$ is Brownian motion. We may estimate the right-hand side of (5.38) by using the fact that

$$
\exp \left[\lambda \int_{0}^{t} e^{A s} d W(s)-\lambda^{2}\left[e^{2 A t}-1\right] / 4 A\right]
$$

is a martingale for any $\lambda \in \mathbb{R}$. We conclude that

$$
\int_{T-\Delta}^{T} \rho(t) d t \leq \exp \left[-\frac{z^{2}}{16 \varepsilon \Delta}\right], \quad z>2 \eta .
$$

From (5.35) and (5.39) applied to (5.36) we can see now that there is a universal constant $C_{5}$ such that

$$
G(z, 0, T-\Delta, T) \leq \frac{C_{5}}{\sqrt{2 \pi \varepsilon \Delta}} \exp \left[\frac{-z^{2}}{2 C_{5} \varepsilon \Delta}\right], \quad|z|>\eta / 2 .
$$

The estimates (5.35) and (5.40) may be substituted into the right-hand side of (5.30) to obtain the inequality

$$
\begin{aligned}
G(y, 0,0, T)^{1+\alpha} \leq & \sup _{|z|<\eta / 2}\left\{\exp \left[-\frac{(y-z)^{2}}{2 \varepsilon(T-\Delta)}\right] F_{0}(y, z)\right\} \frac{1}{\sqrt{2 \pi \varepsilon(T-\Delta)}} \\
& \cdot \int_{-\eta / 2}^{\eta / 2} G(z, 0, T-\Delta, T)^{1+\alpha} d z \\
+ & \exp \left[-K^{2} / C_{6}\right] \int_{-\infty}^{\infty} \frac{d z}{\sqrt{2 \pi \varepsilon(T-\Delta)}} \exp \left[-\frac{(y-z)^{2}}{2 \varepsilon(T-\Delta)}\right] \\
& \cdot F_{0}(y, z) \frac{C_{6}}{(2 \pi \varepsilon \Delta)^{(1+\alpha) / 2}} \exp \left[-\frac{z^{2}}{2 C_{6} \varepsilon \Delta}\right],
\end{aligned}
$$

where $C_{6}$ is a universal constant. The second term on the right-hand side of (5.41) is a Gaussian integral and so can be explicitly evaluated. 
To estimate the first term we use (5.35) and the inequality

$$
(a+b)^{1+\alpha} \leq a^{1+\alpha}+2^{\alpha}(1+\alpha) a^{\alpha} b+2^{1+\alpha} b^{1+\alpha}, \quad a, b>0,
$$

in the integration over the interval $[-\eta / 2, \eta / 2]$. From (5.41) one sees then that $(5.15)$ holds for a constant $C=C^{\prime}|\log (A T)|$ where $C^{\prime}$ is universal. Hence, as for the lower bound, we may conclude that (5.15) holds for some universal $C$ provided $A T<\delta$ with $\delta>0$ also universal.

We can use the methodology of Lemma 5.2 to obtain similar estimates on $G(y, \xi, 0, T)$ for all $\xi \in \mathbb{R}$. To motivate the estimates we shall obtain, consider the linear case $b(y, s)=A(s) y$ for which

$$
G(y, \xi, 0, T)=\frac{1}{\sqrt{2 \pi \varepsilon \sigma^{2}(T)}} \exp \left[-\frac{(\xi-\Lambda(T) y)^{2}}{2 \varepsilon \sigma^{2}(T)}\right],
$$

where $\Lambda(T)$ and $\sigma^{2}(T)$ are as in (5.1). Observe now that

$$
y-\xi / \Lambda(T)=y+\int_{0}^{T} b(\xi, s) d s-\xi+O\left[(A T)^{2}\right] \xi .
$$

It follows that, provided $A T \leq 1$, there is a positive universal constant $C$ such that

$$
[y-\xi / \Lambda(T)]^{2} \leq\left[y+\int_{0}^{T} b(\xi, s) d s-\xi\right]^{2}(1+C A T)+C(A T)^{3} \xi^{2} .
$$

Lemma 5.3. Suppose $b(\cdot, \cdot)$ satisfies $(1.1)$ and, in addition, $b(0, \cdot) \equiv 0$. Then there are universal positive constants $\delta$ and $C$ such that the Green's function $G$ defined by (1.4) satisfies the inequalities

$$
\begin{aligned}
& G(y, \xi, 0, T) \\
& \quad \leq \frac{1}{\sqrt{2 \pi \varepsilon T}} \exp \left[-\frac{\left\{y+\int_{0}^{T} b(\xi, s) d s-\xi\right\}^{2}}{2 \varepsilon T(1+C A T)}+\frac{C(A T)^{3} \xi^{2}}{2 \varepsilon T}+C A T\right],
\end{aligned}
$$

and

(5.43) $\quad G(y, \xi, 0, T)$

$$
\geq \frac{1}{\sqrt{2 \pi \varepsilon T}} \exp \left[-\frac{\left\{y+\int_{0}^{T} b(\xi, s) d s-\xi\right\}^{2}}{2 \varepsilon T}(1+C A T)-\frac{C(A T)^{3} \xi^{2}}{2 \varepsilon T}-C A T\right],
$$

provided $A T \leq \delta$.

Proof. We proceed as in Lemma 5.2. To establish (5.43) we suppose we have already shown that

$$
\begin{aligned}
G(y, \xi, t, T) \geq & \frac{1}{\sqrt{2 \pi \varepsilon(T-t)}} \exp \left[-\frac{\left\{y+\int_{t}^{T} b(\xi, s) d s-\xi\right\}^{2}}{2 \varepsilon(T-t)}\{1+C A(T-t)\}\right. \\
& \left.-\frac{C[A(T-t)]^{3} \xi^{2}}{2 \varepsilon(T-t)}-C A(T-t)\right]
\end{aligned}
$$


for $T-t=T / 2^{N}$, where $N$ is some integer $N \geq 1$. We shall show that for a sufficiently large constant $C>0,(5.44)$ also holds for $T-t=T / 2^{N-1}$. Using (4.4) with $x=\xi$ we obtain an inequality analogous to (5.18). Thus on setting $T-t_{N}=$ $T / 2^{N}$ for $N \in \mathbb{N}$, it follows from (4.5) that

$$
\begin{array}{r}
G\left(y, \xi, t_{N-1}, T\right)^{1-\alpha} \geq \int_{-\infty}^{\infty} \frac{1}{\sqrt{2 \pi \varepsilon T / 2^{N}}} \exp \left[-\frac{(y-z)^{2}}{2 \varepsilon T / 2^{N}}\right] F_{N}(y, z) \\
\cdot G\left(z+\int_{t_{N-1}}^{t_{N}} b(\xi, s) d s, \xi, t_{N}, T\right)^{1-\alpha} d z
\end{array}
$$

where similarly to (5.19) one may take

$$
\begin{aligned}
-\log F_{N}(y, z)= & \frac{A^{2}(1-\alpha)}{3 \alpha \varepsilon} \frac{T}{2^{N}}\left[(y-\xi)^{2}+(y-\xi)(z-\xi)+(z-\xi)^{2}\right] \\
& +\frac{A^{2}(1-\alpha)}{\alpha \varepsilon} \int_{t_{N-1}}^{t_{N}} d s\left\{\int_{t_{N-1}}^{s} b\left(\xi, s^{\prime}\right) d s^{\prime}\right\}^{2}+\frac{A^{2}(1-\alpha)}{12 \alpha} \frac{T^{2}}{2^{2 N}} .
\end{aligned}
$$

We change the variable $z$ of integration in (5.45) to $z^{\prime}$ where

$$
z^{\prime}=z+\int_{t_{N-1}}^{T} b(\xi, s) d s-\xi .
$$

From (5.46) we see that

$$
\begin{aligned}
-\log F_{N}(y, z) \leq & \frac{A^{2}(1-\alpha)}{\alpha \varepsilon} \frac{T}{2^{N}}\left[\left\{y+\int_{t_{N-1}}^{T} b(\xi, s) d s-\xi\right\}^{2}+z^{\prime 2}\right] \\
& +\frac{3 A^{2}(1-\alpha)}{\alpha \varepsilon} \frac{T}{2^{N}}\left[\frac{A T \xi}{2^{N}}\right]^{2}+\frac{A^{2}(1-\alpha)}{12 \alpha} \frac{T^{2}}{2^{2 N}} .
\end{aligned}
$$

Using the variable $z^{\prime}$ of (5.47) and (5.48) we may argue as in the proof of Lemma 5.2 that (5.44) holds for $t=t_{N-1}$ with constant $C_{N-1}=5 C_{N} / 8+K$ for some universal constant $K$, where $C_{N}$ is the constant in (5.44) when $t=t_{N}$. Thus we have established (5.43) provided we can show that $\lim _{N \rightarrow \infty} 5^{N} C_{N} / 8^{N}=0$.

As in Lemma 5.2 we shall complete the proof of (5.43) by showing that it holds with a constant $C=C(A T)$ which diverges logarithmically as $T \rightarrow 0$. To see this we observe, as in (5.45), that

$$
\begin{aligned}
G(y, \xi, 0, T)^{1-\alpha} \geq & \int_{-\infty}^{\infty} \frac{1}{\sqrt{2 \pi \varepsilon(T-\Delta)}} \exp \left[-\frac{\left\{y+\int_{0}^{T} b(\xi, s) d s-\xi-z\right\}^{2}}{2 \varepsilon(T-\Delta)}\right] \\
& \cdot F(y, z) G\left(z+\xi-\int_{T-\Delta}^{T} b(\xi, s) d s, \xi, T-\Delta, T\right)^{1-\alpha} d z,
\end{aligned}
$$

where, as in $(5.48)$, we may take $F(y, z)$ to be given by

$$
\begin{aligned}
-\log F(y, z)= & \frac{A^{2}(1-\alpha)}{\alpha \varepsilon} T\left[\left\{y+\int_{0}^{T} b(\xi, s) d s-\xi\right\}^{2}+z^{2}\right] \\
& +\frac{3 A^{2}(1-\alpha)}{\alpha \varepsilon} T[A T \xi]^{2}+\frac{A^{2}(1-\alpha)}{12 \alpha} T^{2} .
\end{aligned}
$$


Let $F(\cdot, \cdot)$ be the function defined from (1.11). Then the function

$$
v(z, t)=G(z+F(\xi, t), \xi, t, T), \quad t<T,
$$

satisfies the terminal value problem

$$
\begin{aligned}
& 0=\frac{\partial v}{\partial t}+[b(z+F(\xi, t), t)-b(F(\xi, t), t)] \frac{\partial v}{\partial z}+\frac{\varepsilon}{2} \frac{\partial^{2} v}{\partial z^{2}}, \quad t<T, z \in \mathbb{R} \\
& \delta(z-\xi)=\lim _{t \rightarrow T} v(z, t), \quad z \in \mathbb{R}
\end{aligned}
$$

From(5.51) we see that we can proceed now exactly as in the proof of Lemma 5.2 by replacing the Green's function on the right-hand side of (5.49) by the solution to (5.51) on the interval $|z|<\eta$ with Dirichlet boundary conditions on $|z|=\eta$. Using the fact that

$$
\left|F(\xi, t)-\left\{\xi-\int_{t}^{T} b(\xi, s) d s\right\}\right| \leq C[A(T-t)]^{2}|\xi|,
$$

for some universal constant $C$, we conclude that the inequality (5.43) holds. The proof of the upper bound (5.42) on the Green's function is obtained in a similar way, following the argument of Lemma 5.2.

Corollary 5.4. Suppose $b(\cdot, \cdot)$ satisfies $(1.1)$ and $b(0, \cdot) \equiv 0$. Then there exist positive universal constants $\eta, C_{3}, \gamma_{3}, C_{4}$, and $\gamma_{4}$ such that (5.4) holds provided $A T \leq \eta$ and $\delta=T / 2$.

Proof. To show the first inequality in (5.4) we consider

$$
\begin{aligned}
P\left(Y_{\varepsilon}(T / 2)<C_{3} y / 2 \mid Y_{\varepsilon}(0)=y, Y_{\varepsilon}(T)=0\right) & \\
= & G(y, 0,0, T)^{-1} \int_{-\infty}^{C_{3} y / 2} d \xi G(y, \xi, 0, T / 2) G(\xi, 0, T / 2, T) .
\end{aligned}
$$

It is easy to see now by using Lemma 5.2 how to bound $G(y, 0,0, T)$ from below and $G(\xi, 0, T / 2, T)$ from above. Using also Lemma 5.3 to bound $G(y, \xi, 0, T / 2)$ from above, we conclude that the first inequality in (5.4) holds for $\delta=T / 2$ provided $\eta>0$ is sufficiently small. To show the second inequality of (5.4) we write

$$
\begin{aligned}
P\left(Y_{\varepsilon}(T / 2)>C_{4} y / 2 \mid Y_{\varepsilon}(0)=y, Y_{\varepsilon}(T)=0\right) & \\
= & G(y, 0,0, T)^{-1} \int_{C_{4} y / 2}^{\infty} G(y, \xi, 0, T / 2) G(\xi, 0, T / 2, T),
\end{aligned}
$$

and argue as in the previous paragraph.

In order to show that (5.4) continues to hold when $\delta / T<<1 / 2$ we need to obtain some further estimates on Green's functions. Towards that goal we strengthen Corollary 5.4 as follows. 
Lemma 5.5. Suppose $b(\cdot, \cdot)$ satisfies $(1.1)$ and $b(0, \cdot) \equiv 0$. Then there exist positive universal constants $\eta, C_{1}$, and $C_{2}$ such that, if $A T \leq \eta$,

$$
P\left(\sup _{0 \leq s \leq T}\left|Y_{\varepsilon}(s)\right|>\rho \mid Y_{\varepsilon}(0)=y, Y_{\varepsilon}(T)=0\right) \leq \exp \left[-C_{1} \rho^{2} / 2 \varepsilon T\right],
$$

provided $|\rho| \geq C_{2}[|y|+\sqrt{\varepsilon T}]$.

Proof. We make a dyadic decomposition of the interval $0 \leq s \leq T$. Thus for $n \in \mathbb{N}$, let $S_{n}$ be defined by $S_{n}=\left\{j T / 2^{n}: 0 \leq j \leq 2^{n}\right\}$. It is evident from the continuity of $Y_{\varepsilon}(\cdot)$ that

$$
\begin{aligned}
& P\left(\sup _{0 \leq s \leq T}\left|Y_{\varepsilon}(s)\right|>\rho \mid Y_{\varepsilon}(0)=y, Y_{\varepsilon}(T)=0\right) \\
& \leq \sum_{n=1}^{\infty} P\left(\sup _{s \in S_{n}-S_{n-1}}\left|Y_{\varepsilon}(s)\right|>\rho\left(1-\mu^{n+1}\right),\right. \\
&\left.\sup _{s \in S_{n-1}}\left|Y_{\varepsilon}(s)\right| \leq \rho\left(1-\mu^{n}\right) \mid Y_{\varepsilon}(0)=y, Y_{\varepsilon}(T)=0\right),
\end{aligned}
$$

provided $\mu \in(0,1)$ satisfies $\rho(1-\mu)>|y|$. Observe next that

$$
\begin{aligned}
& P\left(\sup _{s \in S_{n}-S_{n-1}}\left|Y_{\varepsilon}(s)\right|>\rho\left(1-\mu^{n+1}\right), \sup _{s \in S_{n-1}}\left|Y_{\varepsilon}(s)\right| \leq \rho\left(1-\mu^{n}\right) \mid Y_{\varepsilon}(0)=y, Y_{\varepsilon}(T)=0\right) \\
& \quad \leq \sum_{s \in S_{n}-S_{n-1}} P\left(\left|Y_{\varepsilon}(s)-Y_{\varepsilon}\left(s+T / 2^{n}\right)\right|>\rho \mu^{n}(1-\mu) \mid Y_{\varepsilon}(0)=y, Y_{\varepsilon}(T)=0\right) .
\end{aligned}
$$

The probability in the sum on the right-hand side of (5.54) can be expressed in terms of the Green's function (1.4) as

$$
\begin{gathered}
P\left(\left|Y_{\varepsilon}(s)-Y_{\varepsilon}\left(s+T / 2^{n}\right)\right|>\rho \mu^{n}(1-\mu) \mid Y_{\varepsilon}(0)=y, Y_{\varepsilon}(T)=0\right) \\
=G(y, 0,0, T)^{-1} \int_{-\infty}^{\infty} \int_{-\infty}^{\infty} d \xi d \zeta G(y, \xi, 0, s) H\left(|\xi-\zeta|-\rho \mu^{n}(1-\mu)\right) \\
\cdot G\left(\xi, \zeta, s, s+T / 2^{N}\right) G\left(\zeta, 0, s+T / 2^{n}, T\right),
\end{gathered}
$$

where $H(z), z \in \mathbb{R}$, is the Heaviside function. We may estimate the integral on the right-hand side of (5.55) by using Lemma 5.2 and Lemma 5.3. We first consider the integral with respect to $\xi$ in $(5.55)$ for a fixed $\xi \in \mathbb{R}$. From Lemma 5.3 we have that

$$
\begin{aligned}
& G(y, \xi, 0, s) G\left(\xi, \zeta, s, s+T / 2^{n}\right) \\
& \leq \quad \frac{1}{2 \pi \varepsilon \sqrt{s T / 2^{n}}} \exp \left[-\frac{\left\{y+\int_{0}^{s} b\left(\xi, s^{\prime}\right) d s^{\prime}-\xi\right\}^{2}}{2 \varepsilon s}(1-C A s)\right. \\
& \quad+\frac{C(A s)^{3} \xi^{2}}{2 \varepsilon s}+C A s-\frac{\left\{\xi+\int_{s}^{s+T / 2^{n}} b\left(\zeta, s^{\prime}\right) d s^{\prime}-\zeta\right\}^{2}}{2 \varepsilon T / 2^{n}}\left(1-C A T / 2^{n}\right) \\
& \left.\quad+\frac{C\left(A T / 2^{n}\right)^{3}}{2 \varepsilon T / 2^{n}} \zeta^{2}+C A T / 2^{n}\right] .
\end{aligned}
$$


Setting $z=y+\int_{0}^{s} b\left(\zeta, s^{\prime}\right) d s^{\prime}-\zeta$ we see from (5.56) that

$$
\begin{aligned}
& G(y, \xi, 0, s) G\left(\xi, \zeta, s, s+T / 2^{n}\right) \\
& \leq \frac{1}{2 \pi \varepsilon \sqrt{s T / 2^{n}}} \exp \left[-\frac{\left\{z^{2}+2(\zeta-\xi) z+(\zeta-\xi)^{2}\right\}}{2 \varepsilon s}-\frac{(\zeta-\xi)^{2}}{2 \varepsilon T / 2^{n}}\right. \\
&\left.+\frac{C A}{\varepsilon}\left[(\zeta-\xi)^{2}+z^{2}+\zeta^{2}\right]+C A T\right],
\end{aligned}
$$

for some universal constant $C$. Integrating the right-hand side of (5.57) over the region $|\xi-\zeta|>\rho \mu^{n}(1-\mu)$ we conclude that

$$
\begin{aligned}
\int_{-\infty}^{\infty} d \xi G(y, \xi, 0, s) H\left(|\xi-\zeta|-\rho \mu^{n}(1-\mu)\right) G\left(\xi, \zeta, s, s+T / 2^{n}\right) \\
\leq \exp \left[-\frac{\rho^{2} \mu^{2 n}(1-\mu)^{2}}{4 \varepsilon T / 2^{n}}\right] \frac{1}{\sqrt{2 \pi \varepsilon \tau_{n}}} \\
\quad \cdot \exp \left[-\frac{z^{2}}{2 \varepsilon \tau_{n}}\left\{\frac{1}{2}-\frac{2 C A T}{2^{n}}\right\}+\frac{C A}{\varepsilon}\left\{z^{2}+\zeta^{2}\right\}+C A T\right],
\end{aligned}
$$

where $\tau_{n}=T / 2^{n}+s / 2-2 C A s T / 2^{n}$. Hence if we use the inequalities

$$
(y-\zeta)^{2}[1-A s]-A s \zeta^{2} \leq z^{2} \leq(y-\zeta)^{2}[1+A s]+2 A s \zeta^{2},
$$

which are valid for $A T \leq 1$, and substitute the right-hand side of (5.58) into the right-hand side of (5.55), we may conclude from Lemma 5.2 that the left-hand side of (5.55) is bounded by a Gaussian integral in $\zeta$. Evaluating this integral we have then that

$$
\begin{aligned}
& P\left(\left|Y_{\varepsilon}(s)-Y_{\varepsilon}\left(s+T / 2^{n}\right)\right|>\rho \mu^{n}(1-\mu) \mid Y_{\varepsilon}(0)=y, Y_{\varepsilon}(T)=0\right) \\
& \leq G(y, 0,0, T)^{-1} \frac{\sqrt{2}}{\left[2 \pi \varepsilon\left(T+T / 2^{n}\right)\right]^{1 / 2}} \\
& \quad \cdot \exp \left[-\frac{\rho^{2} \mu^{2 n}(1-\mu)^{2}}{4 \varepsilon T / 2^{n}}-\frac{y^{2}}{2 \varepsilon\left(T+T / 2^{n}\right)}+\frac{C A y^{2}}{\varepsilon}+C A T\right]
\end{aligned}
$$

for some universal constant $C$. Choosing now $\mu$ in (5.59) to satisfy $1 / \sqrt{2}<\mu<1$ and using the lower bound for $G(y, 0,0, T)$ in Lemma 5.2 we conclude from (5.59) that

$$
\begin{array}{r}
P\left(\left|Y_{\varepsilon}(s)-Y_{\varepsilon}\left(s+T / 2^{n}\right)\right|>\rho \mu^{n}(1-\mu) \mid Y_{\varepsilon}(0)=y, Y_{\varepsilon}(T)=0\right) \\
\leq \exp \left[\frac{-\rho^{2} \mu^{2 n}(1-\mu)^{2}}{8 \varepsilon T / 2^{n}}\right] \quad \text { if } \rho \geq C_{2}[|y|+\sqrt{\varepsilon T}]
\end{array}
$$

provided $C_{2}$ is a sufficiently large universal constant. Hence (5.53) and (5.54) imply

$$
\begin{aligned}
P\left(\sup _{0 \leq s \leq T}\left|Y_{\varepsilon}(s)\right|>\rho \mid Y_{\varepsilon}(0)=y, Y_{\varepsilon}(T)=0\right) \\
\leq \sum_{n=1}^{\infty} 2^{n-1} \exp \left[\frac{-\rho^{2} \mu^{2 n}(1-\mu)}{8 \varepsilon T / 2^{n}}\right] \leq \exp \left[-\frac{C_{1} \rho^{2}}{2 \varepsilon T}\right],
\end{aligned}
$$

for some universal constant $C_{1}>0$. 
To prove (5.4) under the assumptions $(1.1)$ and $b(0, \cdot) \equiv 0$ we actually need versions of Lemma 5.3 and Lemma 5.5 which hold in the situation when $b(0, \cdot) \not \equiv 0$. A slight modification of the proof of Lemma 5.3 yields:

Corollary 5.6. Suppose $b(\cdot, \cdot)$ satisfies (1.1). Then there are universal positive constants $\eta$ and $C$ such that the Green's function $G$ defined by (1.4) satisfies the inequalities

$$
\begin{aligned}
G(y, \xi, 0, T) \leq & \frac{1}{\sqrt{2 \pi \varepsilon T}} \exp \left[-\frac{\left\{y+\int_{0}^{T} b(\xi, s) d s-\xi\right\}^{2}}{2 \varepsilon T(1+C A T)}\right. \\
& \left.+\frac{C(A T)^{3} \xi^{2}}{2 \varepsilon T}+C A T+\frac{C A}{\varepsilon}\left\{\int_{0}^{T}|b(0, s)| d s\right\}^{2}\right], \\
G(y, \xi, 0, T) \geq & \frac{1}{\sqrt{2 \pi \varepsilon T}} \exp \left[-\frac{\left\{y+\int_{0}^{T} b(\xi, s) d s-\xi\right\}^{2}}{2 \varepsilon T}(1+C A T)\right. \\
& \left.-\frac{C(A T)^{3} \xi^{2}}{2 \varepsilon T}-C A T-\frac{C A}{\varepsilon}\left\{\int_{0}^{T}|b(0, s)| d s\right\}^{2}\right],
\end{aligned}
$$

provided $A T \leq \eta$.

We can also slightly modify the proof of Lemma 5.5 to obtain the following.

Corollary 5.7. Suppose $b(\cdot, \cdot)$ satisfies (1.1). Then for any $y \in \mathbb{R}$ which satisfies

$$
|y|+\sqrt{\varepsilon T} \geq \int_{0}^{T}|b(0, s)| d s,
$$

the result of Lemma 5.5 holds.

Proof. We simply use the Green's functions bounds of Corollary 5.6 in place of the bounds of Lemma 5.3 in the argument proving Lemma 5.5.

Lemma 5.8. Suppose $b(\cdot, \cdot)$ satisfies $(1.1)$ and $b(0, \cdot) \equiv 0$. For $\lambda \in \mathbb{R}$ define $b_{\lambda}(\cdot, \cdot)$ by $b_{\lambda}(y, s)=b(y+\lambda s, s)$ for $y \in \mathbb{R}$ and $0 \leq s \leq T$, and let $G_{\lambda}$ be the Green's function (1.4) associated with $b_{\lambda}$. Then there are universal positive constants $\eta$ and $C$ such that the following inequalities hold provided $A T \leq \eta$ :

$$
\begin{aligned}
& \frac{G_{\lambda}(y, 0,0, T)}{G_{0}(y, 0,0, T)} \leq \exp \left[\frac{C|\lambda| A T}{\varepsilon}\left\{|y|+|\lambda| A T^{2}\right\}+C A T\right], \\
& \frac{G_{\lambda}(y, 0,0, T)}{G_{0}(y, 0,0, T)} \geq \exp \left[-\frac{C|\lambda| A T}{\varepsilon}\left\{|y|+|\lambda| A T^{2}\right\}-C A T\right] .
\end{aligned}
$$

Proof. Consider first the situation when $|y| \leq|\lambda| A T^{2}$. The result follows from Corollary 5.6 upon using the inequality $\int_{0}^{T}\left|b_{\lambda}(0, s)\right| d s \leq|\lambda| A T^{2} / 2$, whence we need only prove (5.65) and (5.66) for $|y| \geq|\lambda| A T^{2}$. Observe now that if $|y|=O(\sqrt{\varepsilon T})$ then $|\lambda| A T|y| / \varepsilon=O\left(y^{2} / \varepsilon T\right)=O(1)$. Hence we might expect to prove $(5.65)$ 
and (5.66) for $|y|=O(\sqrt{\varepsilon T})$ by perturbation methods. To implement this we consider the function $u_{\lambda}(z, t), z \in \mathbb{R}, t<T$, defined by

$$
u_{\lambda}(z, t)=G_{\lambda}\left(z-\int_{t}^{T} b_{\lambda}(0, s) d s, 0, t, T\right) .
$$

Evidently $u_{\lambda}$ is a solution to the terminal value problem

$$
\begin{aligned}
& \frac{\partial u_{\lambda}}{\partial t}+\tilde{b}_{\lambda}(z, t) \frac{\partial u_{\lambda}}{\partial z}+\frac{\varepsilon}{2} \frac{\partial^{2} u_{\lambda}}{\partial z^{2}}=0, \quad z \in \mathbb{R}, t<T \\
& \lim _{t \rightarrow T} u_{\lambda}(z, t)=\delta(z)
\end{aligned}
$$

where $\tilde{b}_{\lambda}(z, t)$ is given by the formula

$$
\tilde{b}_{\lambda}(z, t)=b_{\lambda}\left(z-\int_{t}^{T} b_{\lambda}(0, s) d s, t\right)-b_{\lambda}(0, t) .
$$

Following the argument of Lemma 3.4 we see that the terminal value problem (5.68) on the interval $|z|<\eta$ with Dirichlet boundary conditions can be solved by perturbation expansion for times $0 \leq t<T$ provided

$$
\sup \left\{\left|\tilde{b}_{\lambda}(z, t)\right|:|z|<\eta, 0<t<T\right\}(T / \varepsilon)^{1 / 2}<<1 .
$$

Assuming now that $y$ and $\eta$ satisfy the inequalities

$$
|\lambda| A T^{2} \leq|y| \leq \sqrt{\varepsilon T} /(A T)^{\delta}, \eta=\sqrt{\varepsilon T} /(A T)^{2 \delta},
$$

it is clear that the perturbation expansion converges provided $\delta<1 / 2$ and $A T$ is smaller than some constant depending only on $\delta$. In fact, letting $G(z, t)$ for $z \in \mathbb{R}$ and $t>0$, be the probability density function for the normal variable with mean 0 and variance $t$ we have that

$$
\begin{aligned}
& \left|u_{\lambda}(z, t)-G(z, \varepsilon(T-t))-\int_{t}^{T} d s \int_{-\eta}^{\eta} d \xi G(z-\xi, \varepsilon(s-t)) \tilde{b}_{\lambda}(\xi, s) \frac{\partial G(\xi, \varepsilon(T-s))}{\partial \xi}\right| \\
& \leq C(A T)^{2-4 \delta} G(z, 2 \varepsilon(T-t)),
\end{aligned}
$$

provided $|z| \leq \sqrt{\varepsilon T} /(A T)^{\delta}$. Here $A T$ needs to be smaller than some constant depending only on $\delta$, and the constant $C$ on the right-hand side of (5.71) also depends on $\delta$. It is easy to see that

$$
\begin{aligned}
& 72) \quad\left|\int_{-\eta}^{\eta} d \xi G(z-\xi, \varepsilon(s-t)) \tilde{b}_{\lambda}(\xi, s) \frac{\partial}{\partial \xi} G(\xi, \varepsilon(T-s))\right| \\
& \leq \frac{1}{2} \int_{-\infty}^{\infty} d \xi G(z-\xi, \varepsilon(s-t)) \frac{A}{\varepsilon(T-s)}\left[3 \xi^{2}+A^{2} \lambda^{2} T^{2}(T-s)^{2}\right] G(\xi, \varepsilon(T-s)) \\
& =\frac{1}{2}\left\{\frac{A^{3} \lambda^{2} T^{2}(T-s)}{\varepsilon}+\frac{3 A(T-s) z^{2}}{\varepsilon(T-t)^{2}}+\frac{3 A(s-t)}{T-t}\right\} G(z, \varepsilon(T-t))
\end{aligned}
$$


Substituting the inequality (5.72) into (5.71) we conclude that $u_{\lambda}(z, t)$ satisfies the inequalities

$$
\begin{aligned}
u_{\lambda}(z, t) \leq & G(z, \varepsilon(T-t))+C(A T)^{2-4 \delta} G(z, 2 \varepsilon(T-t)) \\
& +\left\{\frac{A^{3} \lambda^{2} T^{2}(T-t)^{2}}{4 \varepsilon}+\frac{3 A z^{2}}{4 \varepsilon}+\frac{3 A(T-t)}{4}\right\} G(z, \varepsilon(T-t)), \\
u_{\lambda}(z, t) \geq & G(z, \varepsilon(T-t))-C(A T)^{2-4 \delta} G(z, 2 \varepsilon(T-t)) \\
& -\left\{\frac{A^{3} \lambda^{2} T^{2}(T-t)^{2}}{4 \varepsilon}+\frac{3 A z^{2}}{4 \varepsilon}+\frac{3 A(T-t)}{4}\right\} G(z, \varepsilon(T-t)) .
\end{aligned}
$$

We have shown that (5.73) and (5.74) hold for the function $u_{\lambda}(z, t)$ which satisfies (5.68) on the rectangle $|z|<\eta, 0<t<T$, and with Dirichlet boundary conditions on $|z|=\eta$. It follows that the function $u_{\lambda}(z, t)$ defined by (5.67), also satisfies (5.74) for $|z| \leq \sqrt{\varepsilon T} /(A T)^{\delta}$ and $0<t<T$. From the argument of Lemma 5.2 we see that the upper bound (5.73) continues to hold for the function (5.67) when $|z| \leq \sqrt{\varepsilon T} /(A T)^{\delta}$.

The inequalities (5.65) and (5.66) can be deduced from (5.73) and (5.74) in the case when $y$ lies in the interval $|\lambda| A T^{2} \leq|y| \leq K \sqrt{\varepsilon T}$, where $K \geq 1$ is a constant. The constant $C$ now in (5.65) and (5.66) depends on $K$, and $A T$ must be chosen sufficiently small depending only on $K$. To obtain the lower bound (5.66) we set $\delta=1 / 8, z=y+\int_{0}^{T} b_{\lambda}(0, s) d s$, and $t=0$ in (5.74). Thus we obtain the inequality

$$
G_{\lambda}(y, 0,0, T) \geq G\left(y+\int_{0}^{T} b_{\lambda}(0, s) d s, \varepsilon T\right) \exp \left[-C\left\{\frac{\lambda^{2}(A T)^{3} T}{\varepsilon}+A T e^{K^{2}}\right\}\right]
$$

for some universal constant $C$. Lemma 5.2 implies that $G_{0}(y, 0,0, T)$ satisfies the upper bound

$$
G_{0}(y, 0,0, T) \leq G(y, \varepsilon T) \exp \left[C A T\left(1+K^{2}\right)\right]
$$

for some universal constant $C$. Now (5.66) follows by estimating from below the ratio of the right-hand side of (5.75) to (5.76). The upper bound (5.65) can be similarly obtained from (5.73) and Lemma 5.2.

To complete the proof of the lemma we use induction as we did in the proof of Lemma 5.2. We consider the lower bound (5.66). Observe first that the previous arguments imply that the lower bound

$$
\frac{G_{\lambda}(y, 0, t, T)}{G_{0}(y, 0, t, T)} \geq \exp \left[\frac{-C|\lambda| A T}{\varepsilon}\{|y|+|\lambda| A T(T-t)\}-C A(T-t)\right]
$$

holds for $0 \leq t<T$ if $y$ lies in one of the regions $|y| \leq|\lambda| A T(T-t)$ or $|\lambda| A T(T-t) \leq$ $|y| \leq K \sqrt{\varepsilon(T-t)}$. For the former region the constant $C$ in (5.77) can be chosen in a universal way provided $A T$ is smaller than some universal constant. For the latter region $C$ depends on $K$ and $A T$, and must be taken to be sufficiently small in a way depending only on $K$.

Suppose now we have proved (5.77) for $T-t=T / 2^{N}$ and $y \in \mathbb{R}$, where $N$ is some integer $N \geq 1$ with constant $C=C_{N}$. We show that (5.77) also holds for 
$T-t=T / 2^{N-1}, y \in \mathbb{R}$, with a constant $C_{N-1}$ given in terms of $C_{N}$. To do this we use the inequality

$$
\begin{aligned}
& G_{\lambda}\left(y, 0, t_{N-1}, T\right)^{1-\alpha} \\
& \quad \geq \int_{-\infty}^{\infty} G_{0}\left(y, z, t_{N-1}, t_{N}\right) \exp \left[-\frac{(1-\alpha)}{2 \alpha \varepsilon} \frac{A^{2} \lambda^{2} T^{3}}{2^{N}}\right] G_{\lambda}\left(z, 0, t_{N}, T\right)^{1-\alpha} d z
\end{aligned}
$$

where $T-t_{n}=T / 2^{n}, n=0,1,2, \ldots$, and $0<\alpha<1$. The inequality (5.78) is derived similarly to (5.18).

We assume $y$ in (5.78) satisfies $|y| \geq \max \left[|\lambda| A T\left(T-t_{N-1}\right), K \sqrt{\varepsilon\left(T-t_{N}\right)}\right]$ and set $\alpha=|\lambda| A T^{2} /|y| 2^{N} \leq 1 / 2$. Then, on substituting (5.77) for $t=t_{N}$ into (5.78) we obtain the inequality

$$
\begin{aligned}
& \frac{G_{\lambda}\left(y, 0, t_{N-1}, T\right)^{1-\alpha}}{G_{0}\left(y, 0, t_{N-1}, T\right)^{1-\alpha}} \\
& \geq \exp \left[-\frac{(1-\alpha)|\lambda| A T|y|}{2 \varepsilon} \frac{-C_{N}(1-\alpha)|\lambda|^{2}(A T)^{2} T}{\varepsilon 2^{N}}-\frac{C_{N}(1-\alpha) A T}{2^{N}}\right] \\
& \quad \cdot G_{0}\left(y, 0, t_{N-1}, T\right)^{\alpha} E\left\{G_{0}\left(Y_{\varepsilon}\left(t_{N}\right), 0, t_{N}, T\right)^{-\alpha}\right. \\
& \left.\quad \cdot \exp \left[-\frac{C_{N}(1-\alpha)|\lambda| A T\left|Y_{\varepsilon}\left(t_{N}\right)\right|}{\varepsilon}\right] \mid Y_{\varepsilon}\left(t_{N-1}\right)=y, Y_{\varepsilon}(T)=0\right\},
\end{aligned}
$$

where $Y_{\varepsilon}(\cdot)$ is the solution to (1.5). From Lemma 5.2 we have that

$$
\frac{G_{0}\left(y, 0, t_{N-1}, T\right)}{G_{0}\left(z, 0, t_{N}, T\right)} \geq \exp \left[-\frac{1}{2} \log 2-\frac{y^{2}}{2 \varepsilon T / 2^{N}}-\frac{C A T}{2^{N}}\right], \quad z \in \mathbb{R},
$$

for some universal constant $C$. Since we are assuming that $|y| \geq K \sqrt{\varepsilon\left(T-t_{N}\right)}$, we conclude from (5.80) that, for sufficiently large $K$,

$$
\frac{G_{0}\left(y, 0, t_{N-1}, T\right)^{\alpha}}{G_{0}\left(z, 0, t_{N}, T\right)^{\alpha}} \geq \exp \left[-\frac{|\lambda| A T|y|}{\varepsilon}\right], \quad z \in \mathbb{R} .
$$

To get a lower bound for the right-hand side of (5.79) we are therefore left to estimate from below the expectation

$$
\begin{aligned}
& E\left\{\exp \left[-\frac{C_{N}(1-\alpha)|\lambda| A T\left|Y_{\varepsilon}\left(t_{N}\right)\right|}{\varepsilon}\right] \mid Y_{\varepsilon}\left(t_{N-1}\right)=y, Y_{\varepsilon}(T)=0\right\} \\
& \quad \geq \exp \left[-\frac{C_{N}(1-\alpha)|\lambda| A T}{\varepsilon} E\left\{\left|Y_{\varepsilon}\left(t_{N}\right)\right| \mid Y_{\varepsilon}\left(t_{N-1}\right)=y, Y_{\varepsilon}(T)=0\right\}\right] .
\end{aligned}
$$

We estimate the expectation on the right-hand side of (5.82) by

$$
\begin{aligned}
& E\left[\left|Y_{\varepsilon}\left(t_{N}\right)\right| \mid Y_{\varepsilon}\left(t_{N-1}\right)=y, Y_{\varepsilon}(T)=0\right] \\
& \quad \leq \frac{3|y|}{4}+\int_{|y| / 4}^{\infty} d \rho P\left(\left|Y_{\varepsilon}\left(t_{N}\right)\right|-y / 2|>\rho| Y_{\varepsilon}\left(t_{N-1}\right)=y, Y_{\varepsilon}(T)=0\right) .
\end{aligned}
$$


We have now that

$$
\begin{aligned}
& \text { (5.84) } P\left(\left|Y_{\varepsilon}\left(t_{N}\right)-y / 2\right|>\rho \mid Y_{\varepsilon}\left(t_{N-1}\right)=y, Y_{\varepsilon}(T)=0\right) \\
& =G_{0}\left(y, 0, t_{N-1}, T\right)^{-1} \int_{-\infty}^{\infty} d \xi G_{0}\left(y, \xi, t_{N-1}, t_{N}\right) H(|\xi-y / 2|-\rho) G_{0}\left(\xi, 0, t_{N}, T\right),
\end{aligned}
$$

with $H(\cdot)$ being the Heaviside function. Now, arguing in the same way as we did to obtain (5.60), we conclude from (5.84) that

$$
\begin{aligned}
& P\left(\left|Y_{\varepsilon}\left(t_{N}\right)-y / 2\right|>\rho \mid Y_{\varepsilon}\left(t_{N-1}\right)=y, Y_{\varepsilon}(T)=0\right) \\
& \quad \leq \exp \left[-\frac{\rho^{2}}{2 \varepsilon T / 2^{N}}+\frac{C A y^{2}}{\varepsilon}\right], \quad \text { if } \rho \geq K_{0} \sqrt{\varepsilon\left(T-t_{N}\right)}
\end{aligned}
$$

where $C$ and $K_{0}$ are universal constants. Hence we have that

$$
\begin{aligned}
\int_{|y| / 4}^{\infty} d \rho P\left(\left|Y_{\varepsilon}\left(t_{N}\right)-y / 2\right|>\rho \mid\right. & \left.Y_{\varepsilon}\left(t_{N-1}\right)=y, Y_{\varepsilon}(T)=0\right) \\
\leq & \frac{\varepsilon T}{|y| 2^{N-2}} \exp \left[-\frac{|y|^{2} 2^{N-4}}{2 \varepsilon T}+\frac{C A y^{2}}{\varepsilon}\right],
\end{aligned}
$$

provided the constant $K_{0}$ in (5.85) satisfies $K \geq 4 K_{0}$. Now (5.83) and (5.86) imply that the expectation on the left-hand side of (5.83) is bounded by $4|y| / 5$. Hence it follows from (5.79), (5.81), and (5.82) that we can take $C_{N-1}=4 C_{N} / 5+K_{1}$ for some universal constant $K_{1}$. Thus in order to complete the proof of (5.66) we need to show that $C_{N}$ satisfies $\lim _{N \rightarrow \infty} 4^{N} C_{N} / 5^{N}=0$. To do this we proceed as in the proof of Lemma 5.2 by proving that (5.66) holds with a constant $C=C(A T)$ which diverges logarithmically in $A T$ as $A T \rightarrow 0$.

We have already observed that (5.66) holds for a universal constant $C$ if $|y| \leq$ $|\lambda| A T^{2}$ and for a constant $C$ depending only on $K$ if $|\lambda| A T^{2} \leq|y| \leq K \sqrt{\varepsilon T}$. Hence we shall assume that $|y| \geq \max \left[|\lambda| A T^{2}, K \sqrt{\varepsilon T}\right]$. Analogously to (5.78), the inequality

$$
\begin{aligned}
& G_{\lambda}(y, 0,0, T)^{1-\alpha} \\
& \geq \int_{-\infty}^{\infty} G_{0}(y, z, 0, T-\Delta) \exp \left[-\frac{(1-\alpha)}{2 \alpha \varepsilon} A^{2} \lambda^{2} T^{3}\right] G_{\lambda}(z, 0, T-\Delta, T)^{1-\alpha} d z
\end{aligned}
$$

holds. We set $\alpha=|\lambda| A T^{2} / 2|y|$ in (5.87), whence $0<\alpha \leq 1 / 2$ and the exponential on the right-hand side of (5.87) can be absorbed into the right-hand side of (5.66). As in (5.67) we shall obtain a perturbation expansion of $G_{\lambda}(z, 0, T-\Delta, T)$ by considering the function

$$
\begin{aligned}
& u_{\lambda}(z, t)=G_{\lambda}\left(z+\varphi_{\lambda}(t), 0, t, T\right), \\
& \quad \text { where } \phi_{\lambda}^{\prime}(t)=b_{\lambda}\left(\varphi_{\lambda}(t), t\right), \quad t<T, \varphi_{\lambda}(T)=0 .
\end{aligned}
$$

Then $u_{\lambda}(z, t)$ is a solution to the terminal value problem (5.68) but now with $\operatorname{drift} \tilde{b}_{\lambda}(z, t)$ given by

$$
\tilde{b}_{\lambda}(z, t)=b_{\lambda}\left(z+\varphi_{\lambda}(t), t\right)-b_{\lambda}\left(\varphi_{\lambda}(t), t\right) .
$$


Since $\left|\tilde{b}_{\lambda}(z, t)\right| \leq A|z|$ for $z \in \mathbb{R}$, we may expand the solution of the Dirichlet problem (5.68) by perturbation theory on the rectangle $|z|<\eta, T-\Delta<t<T$, provided $\eta$ and $\Delta$ satisfy

$$
\eta=K_{1} \sqrt{\varepsilon \Delta}, \quad(A \eta)^{2} \Delta=\nu \varepsilon,
$$

where $K_{1}>>1$ and $\nu<<1$. Thus if $u_{\lambda, D}(z, t)$ denotes the solution to this Dirichlet problem we have, as in (5.23), the inequality

$$
\begin{aligned}
u_{\lambda, D}(z, T-\Delta) \geq & \frac{1}{\sqrt{2 \pi \varepsilon \Delta}}\left[\exp \left\{\frac{-z^{2}}{2 \varepsilon \Delta}\right\}-C_{3} \exp \left[-\frac{K_{1}^{2}}{4}\right]\right. \\
& \left.-C_{4}(\rho) \nu^{1 / 2} \exp \left\{-\frac{z^{2}}{2 \varepsilon(1+\rho) \Delta}\right\}\right], \quad|z|<\eta,
\end{aligned}
$$

where $C_{3}$ is a universal constant, $\rho>0$ can be arbitrary, and $C_{4}(\rho)$ is a constant depending only on $\rho$. We choose now $\nu, K_{1}$, and $\Delta / T$ by

$$
\begin{aligned}
K_{1} & =(A T)^{-k_{1}} \exp \left[C_{1} A y^{2} / \varepsilon\right], \quad \nu^{1 / 2}=(A T)^{k_{2}} \exp \left[-C_{2} A y^{2} / \varepsilon\right], \\
\Delta / T & =(A T)^{k_{1}+k_{2}-1} \exp \left[-\left(C_{1}+C_{2}\right) A y^{2} / \varepsilon\right],
\end{aligned}
$$

where $k_{1}, k_{2}, C_{1}, C_{2}>0$ and $k_{1}+k_{2}>1$. Evidently the choice of $K_{1}, \nu$, and $\Delta$ in (5.92) is consistent with (5.90).

To estimate from below the left-hand side of (5.66) we use the inequality

$$
\begin{aligned}
& G_{\lambda}(y, 0,0, T)^{1-\alpha} \geq \exp \left[-\frac{(1-\alpha)|\lambda| A T|y|}{\varepsilon}\right] \\
& \quad \cdot \int_{-\eta+\varphi_{\lambda}(T-\Delta)}^{\eta+\varphi_{\lambda}(T-\Delta)} G_{0}(y, z, 0, T-\Delta) u_{\lambda, D}\left(z-\varphi_{\lambda}(T-\Delta), T-\Delta\right)^{1-\alpha} d z
\end{aligned}
$$

derived from (5.87) and (5.88). If we substitute now the right-hand side of (5.91) into the right-hand side of (5.93) we obtain an integral which we would like to show is comparable to $G_{0}(y, 0,0, T)^{1-\alpha}$. To do this we write

$$
G_{0}(y, 0,0, T)=\int_{-\infty}^{\infty} G_{0}(y, z, 0, T-\Delta) G_{0}(z, 0, T-\Delta, T) d z
$$

and use perturbation analysis to show that $G_{0}(z, 0, T-\Delta, T)$ is comparable to the right-hand side of (5.91). Using the upper bound (5.35) on $G_{0}(z, 0, T-\Delta, T)$ in (5.94) we obtain an upper bound on $G_{0}(y, 0,0, T)$ which has the same form as the integral on the right-hand side of (5.93).

We compare the principal terms of these integrals. Thus for the integral on the right-hand side of (5.93) the principal term is

$$
\begin{aligned}
(2 \pi \varepsilon \Delta)^{\alpha / 2} \int_{-\eta+\varphi_{\lambda}(T-\Delta)}^{\eta+\varphi_{\lambda}(T-\Delta)} & \frac{d z}{\sqrt{2 \pi \varepsilon \Delta}} G_{0}(y, z, 0, T-\Delta) \\
& \cdot \exp \left[-(1-\alpha) \frac{\left\{z-\varphi_{\lambda}(T-\Delta)\right\}^{2}}{2 \varepsilon \Delta}\right] .
\end{aligned}
$$


For the integral on the right-hand side of (5.94) the principal term is

$$
\int_{-\infty}^{\infty} G_{0}(y, z, 0, T-\Delta) \frac{1}{\sqrt{2 \pi \varepsilon \Delta}} \exp \left[\frac{-z^{2}}{2 \varepsilon \Delta}\right] d z .
$$

Observe now that we may assume $\left|\varphi_{\lambda}(T-\Delta)\right| \leq \eta / 2$. To see this we first note from (5.88) that $\left|\varphi_{\lambda}(T-\Delta)\right| \leq C A|\lambda| T \Delta$ for some universal constant $C$, whence it follows that $\left|\varphi_{\lambda}(T-\Delta)\right| \leq C|y| \Delta / T$. Thus from (5.90) the inequality $\left|\varphi_{\lambda}(T-\Delta)\right| \leq \eta / 2$ will follow if we can show that $2 C|y| \leq K_{1}(T / \Delta)^{1 / 2} \sqrt{\varepsilon T}$, which is equivalent to showing that $4 C^{2}|y|^{2} / \varepsilon T \leq K_{1}^{2}(T / \Delta)$. Choosing $K_{1}$ and $T / \Delta$ as in (5.92) we see that this inequality holds provided $3 k_{1}+k_{2}>2$ and $A T$ is sufficiently small, depending only on $C_{1}$ and $C_{2}$. Similarly we have that

$$
\begin{aligned}
\frac{\varphi_{\lambda}(T-\Delta)^{2}}{2 \varepsilon \Delta} & +\frac{\eta\left|\varphi_{\lambda}(T-\Delta)\right|}{\varepsilon \Delta} \leq \frac{C^{2} A^{2}|\lambda|^{2} T^{2} \Delta}{2 \varepsilon}+\frac{C \eta A|\lambda| T}{\varepsilon} \\
\leq & \frac{C^{2} A^{2}|\lambda|^{2} T^{3}}{2 \varepsilon}+\frac{C K_{1}|y|}{\sqrt{\varepsilon T}}\left(\frac{\Delta}{T}\right)^{1 / 2} \leq \frac{C^{2} A^{2}|\lambda|^{2} T^{3}}{2 \varepsilon}+C^{\prime} A T
\end{aligned}
$$

provided the constants in (5.92) satisfy $k_{2}>k_{1}+5, C_{2}>C_{1}$. In that case the constant $C^{\prime}$ in (5.97) depends only on $k_{1}, k_{2}, C_{1}$, and $C_{2}$. We conclude then that the expression in (5.95) is bounded below by

$$
\begin{aligned}
(2 \pi \varepsilon \Delta)^{\alpha / 2} \exp [ & \left.-\frac{C|\lambda|^{2} A^{2} T^{3}}{\varepsilon}-C A T\right] \\
& \cdot \int_{-\eta / 2}^{\eta / 2} G_{0}(y, z, 0, T-\Delta) \exp \left[-\frac{z^{2}}{2 \varepsilon \Delta}\right] \frac{d z}{\sqrt{2 \pi \varepsilon \Delta}},
\end{aligned}
$$

for a constant $C$ depending only on the constants in (5.92).

Next we bound the integral in (5.98) from below by a constant times the integral in (5.96). To show this we use Lemma 5.3. Thus the the inequalities

$$
\begin{aligned}
& G_{0}(y, z, 0, t) \leq \frac{1}{\sqrt{2 \pi \varepsilon t}} \exp \left[-\frac{(y-z)^{2}}{2 \varepsilon t}+\frac{C A}{\varepsilon}\left(y^{2}+z^{2}\right)+C A t\right] \\
& G_{0}(y, z, 0, t) \geq \frac{1}{\sqrt{2 \pi \varepsilon t}} \exp \left[-\frac{(y-z)^{2}}{2 \varepsilon t}-\frac{C A}{\varepsilon}\left(y^{2}+z^{2}\right)-C A t\right]
\end{aligned}
$$

give upper and lower bounds on the Green's function $G_{0}(y, z, 0, t)$. Substituting the lower bound of (5.99) into (5.96) we conclude that

$$
\begin{aligned}
\int_{-\infty}^{\infty} G_{0}(y, z, 0, T-\Delta) & \frac{1}{\sqrt{2 \pi \varepsilon \Delta}} \exp \left[-\frac{z^{2}}{2 \varepsilon \Delta}\right] d z \\
& \geq \frac{1}{\sqrt{2 \pi \varepsilon T}} \exp \left[-\frac{y^{2}}{2 \varepsilon T}-\frac{C A}{\varepsilon} y^{2}-C A T\right],
\end{aligned}
$$


for some universal constant $C$. Using the upper bound in (5.99) we also have that

$$
\begin{aligned}
& \int_{|z|>\eta / 2} G_{0}(y, z, 0, T-\Delta) \frac{1}{\sqrt{2 \pi \varepsilon \Delta}} \exp \left[-\frac{z^{2}}{2 \varepsilon \Delta}\right] d z \\
& \quad \leq \exp \left[-\frac{\eta^{2}}{16 \varepsilon \Delta}\right] \int_{-\infty}^{\infty} G_{0}(y, z, 0, T-\Delta) \frac{1}{\sqrt{2 \pi \varepsilon \Delta}} \exp \left[-\frac{z^{2}}{4 \varepsilon \Delta}\right] d z \\
& \leq \frac{1}{\sqrt{2 \pi \varepsilon T}} \exp \left[-\frac{\eta^{2}}{16 \varepsilon \Delta}-\frac{y^{2}}{2 \varepsilon T}+\frac{C A}{\varepsilon} y^{2}+C A T+\frac{1}{2} \log 2\right]
\end{aligned}
$$

for some universal constant $C$. Observe that in (5.101) we are assuming that the constants in (5.92) satisfy $k_{1}+k_{2}>2$ so that $\Delta / T \leq A T$. Now taking $\eta$ to be given by (5.90) and (5.92), we conclude from (5.100) and (5.101) that

$$
\begin{aligned}
& \int_{|z|>\eta / 2} G_{0}(y, z, 0, T-\Delta) \frac{1}{\sqrt{2 \pi \varepsilon \Delta}} \exp \left[-\frac{z^{2}}{2 \varepsilon \Delta}\right] d z \\
& (5.102) \leq \exp \left[-\frac{1}{32(A T)^{2 k_{1}}}\right] \int_{-\infty}^{\infty} G_{0}(y, z, 0, T-\Delta) \frac{1}{\sqrt{2 \pi \varepsilon \Delta}} \exp \left[-\frac{z^{2}}{2 \varepsilon \Delta}\right] d z
\end{aligned}
$$

It follows that (5.95) is bounded below by

$$
\begin{array}{r}
(2 \pi \varepsilon \Delta)^{\alpha / 2} \exp \left[-\frac{C|\lambda|^{2} A^{2} T^{3}}{\varepsilon}-C A T\right]\left\{1-\exp \left[-\frac{1}{32(A T)^{2 k_{1}}}\right]\right\} \\
\int_{-\infty}^{\infty} G_{0}(y, z, 0, T-\Delta) \frac{1}{\sqrt{2 \pi \varepsilon \Delta}} \exp \left[-\frac{z^{2}}{2 \varepsilon \Delta}\right] d z .
\end{array}
$$

The integral in (5.103) is the principle term in the expression (5.94) for $G_{0}(y, 0$, $0, T)$. Assuming then that we can replace the integral by $G_{0}(y, 0,0, T)$ and that we take into account only the principal term for $u_{\lambda, D}$ in $(5.93)$, we have from $(5.103)$ that the inequality

$$
\begin{aligned}
& G_{\lambda}(y, 0,0, T)^{1-\alpha} \geq\left[(2 \pi \varepsilon \Delta)^{1 / 2} G_{0}(y, 0,0, T)\right]^{\alpha} \\
& \cdot \exp \left[-\frac{C(1-\alpha)|\lambda| A T}{\varepsilon}\left\{|y|+|\lambda| A T^{2}\right\}-C(1-\alpha) A T\right] G_{0}(y, 0,0, T)^{1-\alpha}
\end{aligned}
$$

holds for some universal constant $C$. By Lemma 5.2 we have that

$$
\left[(2 \pi \varepsilon \Delta)^{1 / 2} G_{0}(y, 0,0, T)\right]^{\alpha} \geq \exp \left[-\frac{\alpha}{2} \log (T / \Delta)-\frac{\alpha y^{2}}{2 \varepsilon T}(1+C A T)-\alpha C A T\right]
$$

for some universal constant $C$. Taking $\alpha$ as before to be given by $\alpha=|\lambda| A T^{2} / 2|y| \leq$ $1 / 2$ and using the fact that $|y| \geq K \sqrt{\varepsilon T}$ we see from (5.92) and (5.105) that

$$
\left[(2 \pi \varepsilon \Delta)^{1 / 2} G_{0}(y, 0,0, T)\right]^{\alpha} \geq \exp \left[-(1-\alpha) C A T\left\{\frac{|\lambda y|}{\varepsilon}|\log (A T)|+1\right\}\right]
$$

where the constant $C$ depends only on the constants $C_{1}, C_{2}, k_{1}$, and $k_{2}$ of $(5.92)$ and also $K$. Combining then (5.104) and (5.106), we have obtained a lower bound of the form (5.66) with a constant $C=C(A T)=C^{\prime}|\log (A T)|$. 
To complete the proof of (5.66) with a constant $C=C(A T)=C^{\prime}|\log (A T)|$ we need to estimate the effect of the error terms in (5.35) and (5.91). From (5.35) the main error term in (5.94) is given by

$$
\int_{-\infty}^{\infty} G_{0}(y, z, 0, T-\Delta) C_{2}(\rho) \nu^{1 / 2} \frac{1}{\sqrt{2 \pi \varepsilon \Delta}} \exp \left[-\frac{z^{2}}{2 \varepsilon(1+\rho) \Delta}\right] d z,
$$

where $\nu$ is given by (5.92). It is evident that by choosing $k_{2}$ and $C_{2}$ sufficiently large in a universal way in (5.92) that the integral of (5.107) is bounded above by $A T$ times the integral on the left-hand side of (5.100). We can similarly estimate the error terms in (5.93) of $u_{\lambda, D}$. If we use the inequality (5.26), then from (5.91) we obtain a term like (5.107). Hence (5.66) with a constant $C=C(A T)=C^{\prime}|\log (A T)|$ holds. By the preceding argument it follows then that (5.66) holds with some universal constant $C$ provided $A T \leq \eta$, where $\eta$ may also be chosen in a universal way.

The completion of the proof of the upper bound (5.65) can be carried out in a way similar to that used to prove the lower bound.

Lemma 5.9. Suppose $b(\cdot, \cdot)$ satisfies (1.1) and $b(0, \cdot) \equiv 0$. If $G$ is the Green's function defined by (1.4), then there are universal positive constants $\eta$ and $C$ such that $G$ satisfies the inequalities,

$$
\frac{G(y, \xi, 0, T)}{G(y, 0,0, T)} \leq \exp \left[-\frac{\xi^{2}}{2 \varepsilon T}(1-C A T)+\frac{\xi y}{\varepsilon T}[1+C A T \operatorname{sgn}(\xi y)]+C A T\right]
$$

and

$$
\frac{G(y, \xi, 0, T)}{G(y, 0,0, T)} \geq \exp \left[-\frac{\xi^{2}}{2 \varepsilon T}(1+C A T)+\frac{\xi y}{\varepsilon T}[1-C A T \operatorname{sgn}(\xi y)]-C A T\right]
$$

for all $y, \xi \in \mathbb{R}$, provided $A T \leq \eta$.

Proof. The result follows from Lemma 5.3 if $|y| \leq|\xi|$, so we shall assume that $|\xi| \leq|y|$. Letting $w(z, t)=G(z, \xi, t, T), t<T$, it follows from (1.2) that the function $w_{\lambda}(z, t)$ defined by

$$
w_{\lambda}(z, t)=\exp \left[-\frac{\lambda z}{\varepsilon}+\frac{\lambda^{2}}{2 \varepsilon}(T-t)\right] w(z+\lambda t, t)
$$

is the solution to the terminal value problem

$$
\begin{aligned}
& 0=\frac{\partial w_{\lambda}}{\partial t}+b(z+\lambda t, t) \frac{\partial w_{\lambda}}{\partial z}+\frac{\varepsilon}{2} \frac{\partial^{2} w_{\lambda}}{\partial z^{2}}+\frac{\lambda}{\varepsilon} b(z+\lambda t, t) w_{\lambda}, \\
& \lim _{t \rightarrow T} w_{\lambda}(z, t)=\exp \left[-\frac{\lambda z}{\varepsilon}\right] \delta(z+\lambda T-\xi) .
\end{aligned}
$$

Taking $\lambda=\xi / T$ in (5.111) we see from Lemma 5.8 that

$$
w_{\lambda}(y, 0)=G_{\lambda}(y, 0,0, T)
$$

$$
\text { . } E\left[\exp \left\{\frac{\lambda}{\varepsilon} \int_{0}^{T} b\left(Y_{\varepsilon, \lambda}(s)+\lambda s, s\right) d s\right\} \mid Y_{\varepsilon, \lambda}(0)=y, Y_{\varepsilon, \lambda}(T)=0\right],
$$


where $Y_{\varepsilon, \lambda}(\cdot)$ is the solution to (1.4) with the drift $b_{\lambda}$ of Lemma 5.8 in place of $b$. Since

$$
\int_{0}^{T}\left|b_{\lambda}(0, s)\right| d s \leq A T^{2}|\lambda| / 2 \leq|\xi| \leq|y|
$$

if $A T \leq 2$, we can use Corollary 5.7 to estimate the expectation in (5.112). To see this first observe that the expectation is bounded above by

$$
\exp \left[\frac{\lambda^{2} A T^{2}}{2 \varepsilon}\right] E\left[\exp \left\{\frac{A T|\lambda|}{\varepsilon} \sup _{0 \leq s \leq T}\left|Y_{\varepsilon, \lambda}(s)\right|\right\} \mid Y_{\varepsilon, \lambda}(0)=y, Y_{\varepsilon, \lambda}(T)=0\right]
$$

To bound the expectation in (5.113) we use the identity

$$
E\left[e^{X}\right]=1+\int_{0}^{1} E\left[X e^{k X}\right] d k
$$

valid for any random variable $X$. From (5.52) we have that

$$
\begin{aligned}
E\left[\sup _{0 \leq s \leq T}\left|Y_{\varepsilon, \lambda}(s)\right| \exp \left\{r \sup _{0 \leq s \leq T}\left|Y_{\varepsilon, \lambda}(s)\right|\right\} \mid Y_{\varepsilon, \lambda}(0)=y, Y_{\varepsilon, \lambda}(T)=0\right] \\
\leq C_{2}[|y|+\sqrt{\varepsilon T}] \exp \left\{r C_{2}[|y|+\sqrt{\varepsilon T}]\right\} \\
\quad \cdot \sum_{n=0}^{\infty}(n+1) \exp \left\{n r C_{2}[|y|+\sqrt{\varepsilon T}]-C_{1} C_{2}^{2} n^{2}[|y|+\sqrt{\varepsilon T}]^{2} / 2 \varepsilon T\right\},
\end{aligned}
$$

for any $r \geq 0$. Assuming $r \leq A T|\lambda| / \varepsilon$ and using the fact that $|\lambda| \leq|y| / T$, we see that there is an integer $n_{0} \geq 1$, depending only on $A T, C_{1}$, and $C_{2}$, such that $2 r \leq C_{1} C_{2} n_{0}[|y|+\sqrt{\varepsilon T}] / 2 \varepsilon T$. Hence (5.115) implies that there is a constant $C$ depending only on $A T$ such that

$$
\begin{aligned}
& E\left[\sup _{0 \leq s \leq T}\left|Y_{\varepsilon, \lambda}(s)\right| \exp \left\{r \sup _{0 \leq s \leq T}\left|Y_{\varepsilon, \lambda}(s)\right|\right\} \mid Y_{\varepsilon, \lambda}(0)=y, Y_{\varepsilon, \lambda}(T)=0\right] \\
& \quad \leq C[|y|+\sqrt{\varepsilon T}] \exp \{C r[|y|+\sqrt{\varepsilon T}]\}, \quad 0 \leq r \leq A T|\lambda| / \varepsilon .
\end{aligned}
$$

It follow now from (5.116), on using the inequality $2 \sqrt{\varepsilon T} \leq \varepsilon /|\lambda|+|\lambda| T$, that there is a constant $C$ depending only on $A T$ such that

$$
\begin{array}{r}
E\left[\exp \left\{\frac{\lambda}{\varepsilon} \int_{0}^{T} b\left(Y_{\varepsilon, \lambda}(s)+\lambda s, s\right) d s\right\} \mid Y_{\varepsilon, \lambda}(0)=y, Y_{\varepsilon, \lambda}(T)=0\right] \\
\leq \exp \left[\frac{C|\lambda| A T}{\varepsilon}\{|y|+|\lambda| T\}+C A T\right] .
\end{array}
$$

Substituting (5.117) into (5.112) and using the inequality (5.65) of Lemma 5.8, we conclude that the upper bound (5.108) holds. The lower bound (5.109) can be established by a similar argument. 
Proof of Theorem 1.2. Since in Corollary 5.4 we already proved the result for $\delta \sim T / 2$ we shall be concerned here with the situation where $\delta / T<<1$. We have now with $y<0$, the identity

$$
\begin{aligned}
& P\left(Y_{\varepsilon}(T-\delta)<C_{3} \delta y / T \mid Y_{\varepsilon}(0)=y, Y_{\varepsilon}(T)=0\right) \\
& \quad=G(y, 0,0, T)^{-1} \int_{-\infty}^{C_{3} \delta y / T} d \xi G(y, \xi, 0, T-\delta) G(\xi, 0, T-\delta, T) .
\end{aligned}
$$

The identity

$$
G(y, 0,0, T)=\int_{-\infty}^{\infty} d \xi G(y, \xi, 0, T-\delta) G(\xi, 0, T-\delta, T)
$$

also holds. From Lemma 5.2 and Lemma 5.9 one obtains from (5.119) the inequality

$$
\begin{aligned}
G(y, 0,0, T) \geq & \frac{G(y, 0,0, T-\delta)}{\sqrt{2 \pi \varepsilon \delta}} \int_{-\infty}^{\infty} d \xi \exp \left[\frac{-\xi^{2}}{2 \varepsilon(T-\delta)}\right. \\
& \left.-\frac{\xi^{2}}{2 \varepsilon \delta}+\frac{\xi y}{\varepsilon(T-\delta)}-\frac{C A \xi^{2}}{\varepsilon}-\frac{C A|\xi y|}{\varepsilon}-C A T\right]
\end{aligned}
$$

for some universal constant $C$. Now let $X$ be the normal variable with mean $\delta y / T$ and variance $\varepsilon \delta(T-\delta) / T$. Then (5.120) is equivalent to

$$
\begin{aligned}
G(y, 0,0, T) \geq & G(y, 0,0, T-\delta)\left(\frac{T-\delta}{T}\right)^{1 / 2} \exp \left[\frac{\delta y^{2}}{2 \varepsilon T(T-\delta)}-C A T\right] \\
& \cdot E\left[\exp \left\{\frac{-C A X^{2}}{\varepsilon}-\frac{C A|y||X|}{\varepsilon}\right\}\right] .
\end{aligned}
$$

Applying Jensen's inequality in (5.121) and then the Schwarz inequality, we conclude that

$$
\begin{aligned}
G(y, 0,0, T) \geq & G(y, 0,0, T-\delta)\left(\frac{T-\delta}{T}\right)^{1 / 2} \\
& \cdot \exp \left[\frac{\delta y^{2}}{2 \varepsilon T(T-\delta)}-C A T-\frac{C A \delta y^{2}}{\varepsilon T}-C A\left(\frac{\delta}{\varepsilon}\right)^{1 / 2}|y|\right]
\end{aligned}
$$

for some universal constant $C$. We similarly have from Lemmas 5.2 and 5.9 that

$$
\begin{aligned}
& \int_{-\infty}^{C_{3} \delta y / T} d \xi G(y, \xi, 0, T-\delta) G(\xi, 0, T-\delta, T) \\
& \leq G(y, 0,0, T-\delta)\left(\frac{T-\delta}{T}\right)^{1 / 2} \exp \left[\frac{\delta y^{2}}{2 \varepsilon T(T-\delta)}+C A T\right] \\
& \cdot E\left[\exp \left\{\frac{C A X^{2}}{\varepsilon}+\frac{C A|y||X|}{\varepsilon}\right\} ; X<\frac{C_{3} \delta y}{T}\right],
\end{aligned}
$$


for some universal constant $C$. Assuming now that $C_{3}>1$, we have then

$$
\begin{aligned}
E[\exp & \left.\left\{\frac{C A X^{2}}{\varepsilon}+\frac{C A|y||X|}{\varepsilon}\right\} ; X<\frac{C_{3} \delta y}{T}\right] \\
\leq & \sqrt{2} \exp \left[-\frac{\left(C_{3}-1\right)^{2} \delta y^{2}}{4 \varepsilon T(T-\delta)}\right] \\
\cdot & E\left[\exp \left\{\frac{C A X_{1}^{2}}{\varepsilon}+\frac{C A|y| X_{1}}{\varepsilon}\right\}+\exp \left\{\frac{C A X_{1}^{2}}{\varepsilon}-\frac{C A|y| X_{1}}{\varepsilon}\right\}\right],
\end{aligned}
$$

where $X_{1}$ is the Gaussian variable with mean $\delta y / T$ and variance $2 \varepsilon \delta(T-\delta) / T$. The expectation on the right-hand side of (5.124) can be computed explicitly. Hence we conclude that, for some universal constant $C$,

$$
\begin{aligned}
E\left[\operatorname { e x p } \left\{\frac{C A X^{2}}{\varepsilon}\right.\right. & \left.\left.+\frac{C A|y||X|}{\varepsilon}\right\} ; X<\frac{C_{3} \delta y}{T}\right] \\
& \leq 2 \sqrt{2} \exp \left[-\frac{\left(C_{3}-1\right)^{2} \delta y^{2}}{4 \varepsilon T(T-\delta)}+\frac{C A \delta y^{2}}{\varepsilon T}+C A \delta\right],
\end{aligned}
$$

The first inequality of (5.4) follows from (5.118)-(5.125) upon taking $C_{3}$ large enough and using the fact that $y<-T \sqrt{\varepsilon / \delta}$.

To prove the second inequality of $(5.4)$ we consider the identity

$$
\begin{aligned}
& P\left(Y_{\varepsilon}(T-\delta)>\frac{C_{4} \delta y}{T} \mid Y_{\varepsilon}(0)=y, Y_{\varepsilon}(T)=0\right) \\
& \quad=G(y, 0,0, T)^{-1} \int_{C_{4} \delta y / T}^{\infty} d \xi G(y, \xi, 0, T-\delta) G(\xi, 0, T-\delta, T) .
\end{aligned}
$$

We now choose $C_{4}$ to satisfy $0<C_{4}<1$ and proceed as before.

\section{Representation formula for the stochastic cost function}

Corollary 4.5 suggests that we can take the limit $\delta \rightarrow 0$ in (3.55) by setting $\lim _{\delta \rightarrow 0} E\left[q_{\varepsilon}\left(x, y_{\varepsilon}(T-\delta), T-\delta\right)\right]=0$, but it does not prove it. In fact Lemma 3.1 shows that $q_{\varepsilon}\left(x, y_{\varepsilon}(T-\delta), T-\delta\right)$ becomes arbitrarily large for $y$ close to $x$ with $y<x$ as $\delta \rightarrow 0$. To deal with this problem we need to obtain a lower bound on $-\partial q_{\varepsilon}(x, y, t) / \partial y$ sharper than in (4.2), in particular one that does not decay as $y \rightarrow-\infty$. In the linear approximation $b(y, s)=A(s) y$, one can express $-\partial q_{\varepsilon}(0, y, 0) / \partial y$ for $y<0$ by the formula,

$$
-\frac{\partial q_{\varepsilon}(0, y, 0)}{\partial y}=\frac{\varepsilon \Lambda(T)}{\sqrt{2 \pi \varepsilon \sigma^{2}(T)}} \exp \left[-\frac{\Lambda(T)^{2} y^{2}}{2 \varepsilon \sigma^{2}(T)}\right] / \Phi\left(\frac{\Lambda(T) y}{\sqrt{\varepsilon \sigma^{2}(T)}}\right),
$$

where $\Phi$ is the cumulative distribution function for the standard normal variable and $\Lambda(T)$ and $\sigma^{2}(T)$ are given by (5.1). Hence provided $A T<1$ we see from (4.12) that

$$
-\frac{\partial q_{\varepsilon}}{\partial y}(0, y, 0) \sim-\frac{\Lambda(T)^{2} y}{\sigma^{2}(T)}, \quad y / \sqrt{\varepsilon T}<<-1
$$


Comparing (6.2) and (4.2), we see that the exponential factor in (4.2) may be removable in the case of nonlinear $b(\cdot, \cdot)$. We prove this in the following lemma.

Lemma 6.1. Suppose $b(\cdot, \cdot)$ satisfies (1.1) and let $q_{\varepsilon}(x, y, t)$ for $x, y \in \mathbb{R}$ and $t<T$, be defined by (1.7). Then there are universal positive constants $C$ and $\eta$ such that

$$
-\frac{\partial q_{\varepsilon}(x, y, t)}{\partial y} \geq\left[\frac{F(x, t)-y}{T-t}\right] e^{-C A(T-t)},
$$

provided $0 \leq t<T, A(T-t)<\eta$, and $y<F(x, t)$, where $F(x, t)$ is the function defined in (1.11).

Proof. From (4.17) we see that

$$
-\frac{\partial q_{\varepsilon}(x, y, t)}{\partial y} \geq e^{-A(T-t)} \frac{\varepsilon G(y, x, t, T)}{\int_{x}^{\infty} G(y, z, t, T) d z} .
$$

Let us assume first that $b(x, s)=0$ for $0 \leq s \leq T$, whence $F(x, t)=x$ for $0 \leq t<T$. From Lemma 5.6 we see that provided $A(T-t)<\eta$ and $\eta$ is chosen sufficiently small,

$$
\begin{aligned}
\frac{\int_{x}^{\infty} G(y, z, t, T) d z}{\varepsilon G(y, x, t, T)} & \leq \frac{1}{\varepsilon} \int_{0}^{\infty} d \xi \exp \left[-\frac{\xi(x-y)}{\varepsilon(T-t)\{1+C A(T-t)\}}\right] \\
& =\frac{(T-t)\{1+C A(T-t)\}}{x-y}
\end{aligned}
$$

where $C$ is a universal constant. The inequality (6.3) follows now from (6.4) and (6.5). To deal with the more general case we make the change of variable as in (5.88) and (5.89), and proceed as above.

Theorem 6.2. Suppose $b(\cdot, \cdot)$ satisfies (1.1) and $q_{\varepsilon}(x, y, t)$ for $x, y \in \mathbb{R}$ and $t<T$, is defined by (1.7). If $\lambda_{\varepsilon}(\cdot, \cdot)$ is the optimal controller defined by (1.16) then for $0 \leq t<T$ and $x, y \in \mathbb{R}$, the functions $q_{\varepsilon}(x, y, t), \partial q_{\varepsilon}(x, y, t) / \partial x$, and $\partial q_{\varepsilon}(x, y, t) / \partial y$ have the representations

$$
\begin{aligned}
& q_{\varepsilon}(x, y, t)=E\left\{\frac{1}{2} \int_{t}^{T}\left[\lambda_{\varepsilon}\left(y_{\varepsilon}(s), s\right)-b\left(y_{\varepsilon}(s), s\right)\right]^{2} d s \mid y_{\varepsilon}(t)=y\right\} \\
& \frac{\partial q_{\varepsilon}(x, y, t)}{\partial y}=-\frac{1}{T-t} \\
& \cdot E\left\{\int_{t}^{T}\left[1+(T-s) \frac{\partial b}{\partial y}\left(y_{\varepsilon}(s), s\right)\right]\left[\lambda_{\varepsilon}\left(y_{\varepsilon}(s), s\right)-b\left(y_{\varepsilon}(s), s\right)\right] d s \mid y_{\varepsilon}(t)=y\right\}, \\
& \text { (6.8) } \frac{\partial q_{\varepsilon}(x, y, t)}{\partial x}=\frac{1}{T-t} \\
& \cdot E\left\{\int_{t}^{T}\left[1-(s-t) \frac{\partial b}{\partial y}\left(y_{\varepsilon}(s), s\right)\right]\left[\lambda_{\varepsilon}\left(y_{\varepsilon}(s), s\right)-b\left(y_{\varepsilon}(s), s\right)\right] d s \mid y_{\varepsilon}(t)=y\right\},
\end{aligned}
$$

where $y_{\varepsilon}(s)$, defined for $t \leq s<T$, is the solution to the SDE (1.14) with initial condition $y_{\varepsilon}(t)=y$. 
Proof. From (3.55) the representation (6.6) for $q_{\varepsilon}(x, y, t)$ holds provided we can show that

$$
\lim _{\delta \rightarrow 0} E\left[q_{\varepsilon}\left(x, y_{\varepsilon}(T-\delta), T-\delta\right) \mid y_{\varepsilon}(t)=y\right]=0 .
$$

In view of Lemma 3.1 and Corollary 4.5, (6.9) will follow if we can show that for $M \geq 1$,

(6.10) $\limsup _{\delta \rightarrow 0} E\left[q_{\varepsilon}\left(x, y_{\varepsilon}(T-\delta), T-\delta\right) ; y_{\varepsilon}(T-\delta) \leq x-M \sqrt{\varepsilon \delta} \mid y_{\varepsilon}(t)=y\right] \leq c(M)$,

where the constant $c(M)$ satisfies $\lim _{M \rightarrow \infty} c(M)=0$. To prove (6.10) we use Lemma 6.1. Thus let $t_{0}<T$ be such that $C A\left(T-t_{0}\right)<1 / 10$, where $C$ is the constant in (6.3). If, in addition, $A\left(T-t_{0}\right)<1 / 10$, then $y_{\varepsilon}(s)$ satisfies the differential inequality

$$
d y_{\varepsilon}(s) \geq\left[\frac{3}{4} \frac{\left\{x-y_{\varepsilon}(s)\right\}}{T-s}-2 \sup _{s \leq s^{\prime} \leq T}\left|b\left(x, s^{\prime}\right)\right|\right] d s+\sqrt{\varepsilon} d W(s),
$$

provided $t_{0} \leq s<T$, and $y_{\varepsilon}(s)<x$. If $t \geq t_{0}$ then we see from (6.11), by following the argument of Lemma 4.3, that, for $\delta /(T-t)<1 / K$,

$$
P\left(y_{\varepsilon}(T-\delta)<x-\rho \mid y_{\varepsilon}(t)=y\right) \leq \exp \left[-\rho^{2} / 20 \varepsilon \delta\right],
$$

provided $\rho \geq K \sqrt{\varepsilon \delta}$ and the constant $K$ depends only on $x$ and $y$. Evidently (6.10) follows from (6.12) on using Lemma 3.1. If $t<t_{0}$ then one can argue as in the proof of Theorem 4.4 that the probability of $y_{\varepsilon}\left(t_{0}\right)$ conditioned on $y_{\varepsilon}(t)=y$ being very negative is extremely small. Then one applies (6.11) for $t_{0} \leq s<T$ to show that (6.10) holds in this case also. We have obtained the representation (6.6).

To prove (6.7) we proceed in a similar way to how we obtained the analogous representation (2.29) in the classical case. Thus let $y_{\varepsilon}(s)$ for $t \leq s<T$, with $y_{\varepsilon}(t)=y$ be as before and for $\Delta y \in \mathbb{R}$ define $y_{\varepsilon, \Delta y}(s)$ by

$$
y_{\varepsilon, \Delta y}(s)=y_{\varepsilon}(s)+(T-s) \Delta y /(T-t), \quad t \leq s<T,
$$

so that $y_{\varepsilon, \Delta y}(t)=y+\Delta y$ and $y_{\varepsilon, \Delta y}(s)$ satisfies the SDE:

$$
d y_{\varepsilon, \Delta y}(s)=\left[\lambda_{\varepsilon}\left(y_{\varepsilon, \Delta y}(s)-(T-s) \Delta y /(T-t), s\right)-\Delta y /(T-t)\right] d s+\sqrt{\varepsilon} d W(s) .
$$

for $t \leq s<T$. Then, by Lemma 3.2, the inequality

$$
+E\left\{\frac{1}{2} \int_{t}^{T-\delta} d s\left[\lambda_{\varepsilon}\left(y_{\varepsilon}(s), s\right)-\frac{\Delta y}{T-t}-b\left(y_{\varepsilon}(s)+(T-s) \frac{\Delta y}{T-t}, s\right)\right]^{2} \mid y_{\varepsilon}(t)=y\right\}
$$

holds, where we have used the fact that (6.13) gives the solution to (6.14). Since, by the argument we used to establish (6.6), one has that

$$
\lim _{\delta \rightarrow 0} E\left\{q_{\varepsilon}\left(x, y_{\varepsilon}(T-\delta)+\delta \Delta y /(T-t), T-\delta\right) \mid y_{\varepsilon}(t)=y\right\}=0
$$


we conclude from (6.15) that

$$
\begin{aligned}
q_{\varepsilon}(x, y+\Delta y, t) \leq E\{ & \frac{1}{2} \int_{t}^{T} d s\left[\lambda_{\varepsilon}\left(y_{\varepsilon}(s), s\right)-\frac{\Delta y}{T-t}\right. \\
& \left.\left.-b\left(y_{\varepsilon}(s)+(T-s) \frac{\Delta y}{T-t}, s\right)\right]^{2} \mid y_{\varepsilon}(t)=y\right\} .
\end{aligned}
$$

To see that the right-hand side of $(6.16)$ is finite, it will be sufficient to show that

$$
E\left\{\int_{t}^{T} \lambda_{\varepsilon}\left(y_{\varepsilon}(s), s\right)^{2} d s \mid y_{\varepsilon}(t)=y\right\}<\infty .
$$

Observe that the inequality (6.17) does not follow in a straightforward way from the fact that $y_{\varepsilon}(s)$ is a solution to $(1.14)$, where $\lambda_{\varepsilon}(\cdot, \cdot)$ is given by (1.16) and $-\partial q_{\varepsilon}(x, y, t) / \partial y$ satisfies (6.3). In fact for $Z_{\varepsilon}(s)$ the solution to $(4.28)$, it is easy to see that

$$
E\left\{\int_{t}^{T} \frac{Z_{\varepsilon}(s)^{2}}{(T-s)^{2}} d s \mid Z_{\varepsilon}(t)=z\right\}=\infty,
$$

for all $\mu>0$. To prove (6.17) we use the fact that the left-hand side of (6.6) is finite. Hence (6.17) follows if we can show that

$$
E\left\{\int_{t}^{T} y_{\varepsilon}(s)^{2} d s \mid y_{\varepsilon}(t)=y\right\}<\infty .
$$

It is easy to see that $(6.18)$ is a consequence of the fact that $\lambda_{\varepsilon}(y, s) \geq b(y, s)$ for $y \in \mathbb{R}$ and $t \leq s<T$, and Lemma 3.4. Here we use the fact that Lemma 3.4 implies that for any $\eta>0, \lambda_{\varepsilon}(y, s)$ is uniformly Lipschitz in $y$ in any region $y \geq x+\eta, t \leq s<T$. Having established (6.17), we obtain from (6.6) and (6.16) the inequality

$$
\begin{aligned}
\limsup _{\Delta y \rightarrow 0} \frac{q_{\varepsilon}(x, y+\Delta y, t)-q_{\varepsilon}(x, y, t)}{\Delta y} \leq & -\frac{1}{T-t} E\left\{\int_{t}^{T}\left[1+(T-s) \frac{\partial b}{\partial y}\left(y_{\varepsilon}(s), s\right)\right]\right. \\
(6.19) & \left.\cdot\left[\lambda_{\varepsilon}\left(y_{\varepsilon}(s), s\right)-b\left(y_{\varepsilon}(s), s\right)\right] d s \mid y_{\varepsilon}(t)=y\right\} .
\end{aligned}
$$

Next, in analogy to $(6.16)$, we have that

$$
\begin{aligned}
q_{\varepsilon}(x, y, t) \leq E\{ & \frac{1}{2} \int_{t}^{T} d s\left[\lambda_{\varepsilon}\left(y_{\varepsilon}(s), s\right)+\frac{\Delta}{T-t}\right. \\
& \left.\left.-b\left(y_{\varepsilon}(s)-(T-s) \frac{\Delta y}{T-t}, s\right)\right]^{2} \mid y_{\varepsilon}(t)=y+\Delta y\right\} .
\end{aligned}
$$

Using now (6.6) with $y$ replaced by $y+\Delta y$ we conclude from (6.20) that

$$
\begin{aligned}
\liminf _{\Delta y \rightarrow 0} \frac{q_{\varepsilon}(x, y+\Delta y, t)-q_{\varepsilon}(x, y, t)}{\Delta y} \geq & -\frac{1}{T-t} E\left\{\int_{t}^{T}\left[1+(T-s) \frac{\partial b}{\partial y}\left(y_{\varepsilon}(s), s\right)\right]\right. \\
(6.21) & \left.\cdot\left[\lambda_{\varepsilon}\left(y_{\varepsilon}(s), s\right)-b\left(y_{\varepsilon}(s), s\right)\right] d s \mid y_{\varepsilon}(t)=y\right\},
\end{aligned}
$$


provided we show that

$$
\begin{aligned}
& \lim _{\eta \rightarrow 0} E\left[\int_{t}^{T}\left|y_{\varepsilon, \eta}(s)-y_{\varepsilon, 0}(s)\right| d s\right]=0, \\
& \lim _{\eta \rightarrow 0} E\left[\int_{t}^{T}\left|\lambda_{\varepsilon}\left(y_{\varepsilon, \eta}(s), s\right)-\lambda_{\varepsilon}\left(y_{\varepsilon, 0}(s), s\right)\right| d s\right]=0,
\end{aligned}
$$

where $y_{\varepsilon, \eta}(s)$ for $t \leq s<T$, is the solution to (1.14) with initial condition $y_{\varepsilon, \eta}(t)=$ $y+\eta$. To prove $(6.22)$ we use the uniform Lipschitz continuity of $\lambda_{\varepsilon}(z, s)$ in any region $z \geq z_{0}, t \leq s \leq T-\delta$, where $\delta>0$ and $z_{0} \in \mathbb{R}$ can be arbitrary. Thus by introducing a stopping time and using the fact that the probability of $y_{\varepsilon, \eta}(s)$ being large and negative is very small we see that

$$
\lim _{\eta \rightarrow 0} E\left[\int_{t}^{T-\delta}\left|y_{\varepsilon, \eta}(s)-y_{\varepsilon, 0}(s)\right| d s\right]=0 .
$$

Now (6.22) follows from (6.18) and (6.24) using the fact that one can obtain a bound in (6.18) which is uniform in $\eta$ for small $\eta$. To prove (6.23) first observe that (6.6) implies that

$$
\sup _{|\eta| \leq \eta_{0}} E\left\{\int_{t}^{T} \lambda_{\varepsilon}\left(y_{\varepsilon, \eta}(s), s\right)^{2} d s\right\}<\infty,
$$

for any $\eta_{0}>0$. Thus it is sufficient to show that

$$
\lim _{\eta \rightarrow 0} E\left[\int_{t}^{T-\delta}\left|\lambda_{\varepsilon}\left(y_{\varepsilon, \eta}(s), s\right)-\lambda_{\varepsilon}\left(y_{\varepsilon, 0}(s), s\right)\right| d s\right]=0
$$

for any $\delta>0$. For any $z_{0} \in \mathbb{R}$ we introduce a stopping time $\tau_{\eta}\left(z_{0}\right)=\inf \{s \geq t$ : $\left.y_{\varepsilon, \eta}(s)=z_{0}\right\}$. From the uniform Lipschitz continuity of $\lambda_{\varepsilon}(z, s)$ in the rectangle $z \geq z_{0}, t \leq s \leq T-\delta$, and (6.24) we have that

$$
\lim _{\eta \rightarrow 0} E\left[\int_{t}^{(T-\delta) \wedge \tau_{\eta}\left(z_{0}\right) \wedge \tau_{0}\left(z_{0}\right)}\left|\lambda_{\varepsilon}\left(y_{\varepsilon, \eta}(s), s\right)-\lambda_{\varepsilon}\left(y_{\varepsilon, 0}(s), s\right)\right| d s\right]=0 .
$$

The expectation in $(6.26)$ exceeds the expectation in $(6.27)$ by at most

$$
2 P\left(\tau_{\eta}\left(z_{0}\right) \wedge \tau_{0}\left(z_{0}\right)<T-\delta\right) \sup _{t \leq t^{\prime}<T-\delta} E\left[\int_{t^{\prime}}^{T-\delta}\left|\lambda_{\varepsilon}\left(y_{\varepsilon}(s), s\right)\right| d s \mid y_{\varepsilon}\left(t^{\prime}\right)=z_{0}\right] .
$$

Since $\lambda_{\varepsilon}(z, s) \geq b(z, s)$ for $z \in \mathbb{R}$ and $t \leq s<T$, the probability in (6.28) decays exponentially fast in $z_{0}$ as $z_{0} \rightarrow-\infty$. In contrast the expectation in (6.28) increases at most linearly in $\left|z_{0}\right|$ as $z_{0} \rightarrow-\infty$. This follows from the representation (6.6) for $q_{\varepsilon}$ and Lemma 3.1. Hence the expression in (6.28) converges to 0 as $z_{0} \rightarrow-\infty$, whence we conclude that (6.26) follows from (6.28). We have proved (6.23). Now (6.7) follows from (6.19) and (6.21). The proof of (6.8) is similar to the proof of (6.7).

Once we have the representations in Theorem 6.2 for $q_{\varepsilon}(x, y, t)$ and its first derivatives, the inequality (1.22) follows easily. 
Corollary 6.3. Suppose the function $b(\cdot, \cdot)$ satisfies the Lipschitz condition (1.1). Then for $x, y \in \mathbb{R}$ and $t<T$, the following inequalities hold:

$$
\begin{aligned}
& \left|\frac{\partial q_{\varepsilon}}{\partial x}(x, y, t)\right| \leq[1+(T-t) A]\left[2 q_{\varepsilon}(x, y, t) /(T-t)\right]^{1 / 2}, \\
& \left|\frac{\partial q_{\varepsilon}}{\partial y}(x, y, t)\right| \leq[1+(T-t) A]\left[2 q_{\varepsilon}(x, y, t) /(T-t)\right]^{1 / 2} .
\end{aligned}
$$

Proof. This follows from Theorem 6.2 on using the representations (6.6), (6.7), and (6.8) by applying the Schwarz inequality to (6.7) and (6.8)).

\section{Proof of Theorem 1.3}

In order to prove convergence as $\varepsilon \rightarrow 0$ of first derivatives in $x$ and $y$ of the function $q_{\varepsilon}(x, y, t)$ defined by $(1.7)$ to the corresponding derivatives of the function $q(x, y, t)$ defined by (1.10), it will generally be necessary to assume the concavity in $y$ of the function $b(y, t)$ in (1.2). Recall however that $q(x, y, t)=0$ if $y \geq F(x, t)$, where $F(\cdot, \cdot)$ is the function defined from (1.11). Thus for $y \geq F(x, t)$ the derivatives of $q(x, y, t)$ are 0 . In this case it follows easily from Corollary 6.3 that the derivatives in $x$ or $y$ of $q_{\varepsilon}(x, y, t)$ converge to 0 as $\varepsilon \rightarrow 0$, without making any further assumptions on the function $b(\cdot, \cdot)$ beyond the Lipschitz condition (1.1).

Corollary 7.1. Suppose $b(\cdot, \cdot)$ satisfies (1.1) and the function $F(\cdot, \cdot)$ is defined from (1.11). Then for $0<\varepsilon \leq 1$ there is a constant $C(x, y, t, T)$ such that

$$
\left|\frac{\partial q_{\varepsilon}}{\partial x}(x, y, t)\right|+\left|\frac{\partial q_{\varepsilon}}{\partial y}(x, y, t)\right| \leq C(x, y, t, T) \varepsilon^{1 / 4},
$$

provided $y \geq F(x, t)$.

Proof. The inequality (7.1) follows from Theorem 1.1 and Corollary 6.3 since $q(x, y, t)=0$ for $y \geq F(x, t)$.

In order to show convergence when $y<F(x, t)$ we shall need to assume $b(\cdot, \cdot)$ is concave and that (1.1) holds. We first prove a result about the classical problem.

Lemma 7.2. For $\alpha \geq 0$, let $y_{\alpha}(s)$, defined for $0 \leq s \leq T$, be the solution of

$$
\frac{d y_{\alpha}}{d s}=b\left(y_{\alpha}(s), s\right)-\alpha \frac{\partial q}{\partial y}\left(x, y_{\alpha}(s), s\right), \quad 0 \leq s<T, y_{\alpha}(0)=y,
$$

where $q(\cdot, \cdot, \cdot)$ is the classical cost function (1.10). Then there is a constant $C(A T)$ depending only on AT such that

$$
0 \leq y_{\alpha}(s)-y_{0}(s) \leq \max [1, \alpha] C(A T) \sqrt{T q(x, y, 0)}, \quad 0 \leq s \leq T .
$$

Proof. We first consider the case $\alpha=1$ since $y_{1}(\cdot)$ is the optimal trajectory for the variational problem (2.2). From (2.2) and (2.14)) we see that, for $0 \leq s<T$,

$$
C_{1}(A T)[q(x, y, 0) / T]^{1 / 2} \leq \frac{d y_{1}(s)}{d s}-b\left(y_{1}(s), s\right) \leq C_{2}(A T)[q(x, y, 0) / T]^{1 / 2}
$$


for some positive constants $C_{1}$ and $C_{2}$ depending only on $A T$. Setting $\varphi_{1}(s)=$ $y_{1}(s)-y_{0}(s)$ it follows from (7.2) and (7.4) that

$$
\left|\varphi_{1}^{\prime}(s)\right| \leq A \varphi_{1}(s)+C_{2}(A T)[q(x, y, 0) / T]^{1 / 2}, \quad 0 \leq s \leq T, \varphi_{1}(0)=0 .
$$

Applying Gronwall's inequality to (7.5) we conclude that (7.3) holds for $\alpha=1$ and a fortiori for $0 \leq \alpha \leq 1$.

Suppose now that $\alpha>1$ in which case $y_{\alpha}(s)>y_{1}(s)$ for $0 \leq s \leq T$. Using the fact that $q(x, y, t)$ is convex in $y$, we see from $(7.2)$ that

$$
\frac{d y_{\alpha}(s)}{d s} \leq b\left(y_{\alpha}(s), s\right)-\alpha \frac{\partial q}{\partial y}\left(x, y_{1}(s), s\right), \quad 0 \leq s<T .
$$

Thus if $\varphi_{\alpha}(s)=y_{\alpha}(s)-y_{0}(s)$ we have that

$$
\left|\varphi_{\alpha}^{\prime}(s)\right| \leq A \varphi_{\alpha}(s)+\alpha C_{2}(A T)[q(x, y, 0) / T]^{1 / 2},
$$

whence (7.3) follows for $\alpha>1$ as before.

We can use the method of Lemma 7.2 to find a region where the paths $y_{\varepsilon}(s)$, defined for $0 \leq s<T$, for the stochastic control problem (1.14) and (1.15) are most likely to be found.

Lemma 7.3. Let $y_{\varepsilon}(s)$, defined for $0 \leq s<T$, be the solution to the stochastic equation (1.14) with $y_{\varepsilon}(0)=y$, where $\lambda_{\varepsilon}(\cdot, \cdot)$ is given by (1.16). Then there is a universal constant $M$ and a constant $C(A T)$ depending only on AT such that

$$
P\left[\inf _{0 \leq s<T}\left[y_{\varepsilon}(s)-y_{0}(s)\right]<-\rho\right] \leq \exp \left[-\rho^{2} / \varepsilon T C(A T)\right]
$$

provided $\rho^{2} \geq M \varepsilon T C(A T)$. There is a further constant $C_{1}(x, y, A, T)$ depending only on $x, y, A$, and $T$ such that

$$
\begin{array}{r}
P\left[\sup _{0 \leq s<T}\left[y_{\varepsilon}(s)-y_{0}(s)\right]>\rho+C(A T) \sqrt{T q(x, y, 0)}+C_{1}(x, y, A, T) \varepsilon^{1 / 4}\right] \\
\leq \exp \left[-\rho^{2} / \varepsilon T C(A T)\right], \quad \text { provided } \rho^{2} \geq M \varepsilon T C(A T) .
\end{array}
$$

Proof. The inequality (7.6) is obtained by using the fact that $y_{\varepsilon}(s) \geq Y_{\varepsilon}(s)$ for $0 \leq s<T$, where $Y_{\varepsilon}(0)=y$ and $Y_{\varepsilon}(\cdot)$ satisfies (1.5). Then one compares solutions of (1.5) to solutions of the deterministic equation (7.2) with $\alpha=0$, using the Lipschitz property $(1.1)$ of $b(\cdot, \cdot)$ and applying Gronwall's inequality.

To obtain the inequality (7.7) we need to use the convexity of the function $q_{\varepsilon}(x, y, s)$ in $y$, which is established in the appendix (Theorem A.2). Let $y_{c}(s)$ for $0 \leq s<T$, be the optimal trajectory $y(\cdot)$ for the variational problem (1.10) with $y(0)=y$. Then, if $y>y_{c}(s)$, we have from Corollary 6.3 that

$$
0 \leq-\frac{\partial q_{\varepsilon}}{\partial y}(x, y, s) \leq-\frac{\partial q_{\varepsilon}}{\partial y}\left(x, y_{c}(s), s\right) \leq(1+A T)\left[\frac{2 q_{\varepsilon}\left(x, y_{c}(s), s\right)}{(T-s)}\right]^{1 / 2}
$$


From Lemma 3.3 we see that there is a constant $C_{2}(x, y, A, T)$ depending only on $x, y, A$, and $T$ such that

$$
q_{\varepsilon}\left(x, y_{c}(s), s\right) \leq q\left(x, y_{c}(s), s\right)+C_{2}(x, y, A, T) \sqrt{\varepsilon}, \quad 0 \leq s<T .
$$

Putting together (7.8) and (7.9)) and using the fact that (7.4) holds for $y_{c}(\cdot)$, we conclude that

$$
\begin{aligned}
0 \leq-\frac{\partial q_{\varepsilon}}{\partial y}(x, y, s) \leq & C_{1}(A T)[q(x, y, 0) / T]^{1 / 2} \\
& +C_{3}(x, y, A, T) \varepsilon^{1 / 4} / \sqrt{T-s}, \quad 0 \leq s<T, y>y_{c}(s)
\end{aligned}
$$

Consider now the diffusion process $Z_{\varepsilon}(\cdot)$ defined as a solution to the stochastic equation

$$
d Z_{\varepsilon}(s)=\mu_{\varepsilon}\left(Z_{\varepsilon}(s), s\right) d s+\sqrt{\varepsilon} d W(s), \quad 0 \leq s<T,
$$

where $\mu_{\varepsilon}(\cdot, \cdot)$ is given by the formula

$\mu_{\varepsilon}(z, s)=\left\{\begin{array}{l}b(z, s)-\frac{\partial q_{\varepsilon}}{\partial y}(x, z, s), \quad z<y_{c}(s), \\ b(z, s)+C_{1}(A T)\left[\frac{q(x, y, 0)}{T}\right]^{1 / 2}+C_{3}(x, y, A, T) \frac{\varepsilon^{1 / 4}}{\sqrt{T-s}}, \quad z>y_{c}(s) .\end{array}\right.$

Then, if $Z_{\varepsilon}(0) \geq y_{\varepsilon}(0)$, it follows from $(7.10)$ that $Z_{\varepsilon}(s) \geq y_{\varepsilon}(s)$ for $0 \leq s<T$, with probability 1 .

For any $t, 0 \leq t<T$, suppose that $z_{0}>y_{c}(t)$ and consider the solution $z(s)$ to the initial value problem

$$
d z(s)=\mu_{\varepsilon}(z(s), s) d s, \quad t \leq s<T, z(t)=z_{0} .
$$

By letting $\varepsilon \rightarrow 0$ in (7.10) we see that $z(s)>y_{c}(s)$ for $t<s \leq T$. Hence on setting $\phi(s)=z(s)-y_{c}(s)$ we have from (7.13) and the Lipschitz property of $b(\cdot, \cdot)$ that, for $t \leq s<T$,

$$
-A \phi(s) \leq \phi^{\prime}(s)
$$

$$
\leq A \phi(s)+C_{1}(A T)[q(x, y, 0) / T]^{1 / 2}+C_{3}(x, y, A, T) \varepsilon^{1 / 4} / \sqrt{T-s} .
$$

Integrating (7.14) we conclude that, fr $t \leq s<T$,

$$
\begin{aligned}
& {\left[z_{0}-y_{c}(t)\right] e^{-A T} \leq z(s)-y_{c}(s)} \\
& \leq e^{A T}\left\{\left[z_{0}-y_{c}(t)\right]+C_{1}(A T)[T q(x, y, 0)]^{1 / 2}+2 \sqrt{T} C_{3}(x, y, A, T) \varepsilon^{1 / 4}\right\} .
\end{aligned}
$$

We can compare the solution of (7.13) to the solution of the stochastic equation (7.11) with initial condition $Z_{\varepsilon}(t)=z_{0}>y_{c}(t)$. Arguing as in the proof of Lemma 3.1 we see that

$$
P\left(\sup _{t \leq s<T}\left|Z_{\varepsilon}(s)-z(s)\right|>\delta\right) \leq \exp \left[-\delta^{2} / \varepsilon T C_{2}(A T)\right],
$$


where the constant $C_{2}(A T)$ depends only on $A T$. Also $\delta$ must satisfy the inequalities

$$
\delta<\left[z_{0}-y_{c}(t)\right] e^{-A T}, \quad \delta^{2} \geq M \varepsilon T C_{2}(A T),
$$

where $M$ is a universal constant. The first inequality in (7.17) ensures by (7.15) that if $\left|Z_{\varepsilon}(s)-z(s)\right|<\delta$ then $Z_{\varepsilon}(s)>y_{c}(s)$. Hence to estimate the probability (7.16) we can assume the drift $\mu_{\varepsilon}(\cdot, \cdot)$ of $(7.11)$ is given by the second formula in $(7.12)$.

To prove (7.7) first observe that the probability in (7.7) is bounded above by the probability

$$
\sup _{0 \leq t<T} P\left[\sup _{t \leq s<T}\left[y_{\varepsilon}(s)-y_{0}(s)\right]>\frac{1+2 e^{A T}}{2\left(1+e^{A T}\right)} \eta \mid y_{\varepsilon}(t)-y_{0}(t)=\frac{\eta}{2\left(1+e^{A T}\right)}\right]
$$

where $\eta$ is given by the formula

$$
\eta=\rho+C(A T) \sqrt{T q(x, y, 0)}+C_{1}(x, y, A, T) \varepsilon^{1 / 4} .
$$

The probability in (7.18) is in turn bounded above by the same probability with $y_{\varepsilon}(s)$ replaced by $Z_{\varepsilon}(s)$. Observe next that

$$
Z_{\varepsilon}(s)-y_{0}(s)>\frac{1+2 e^{A T}}{2\left(1+e^{A T}\right)} \eta \Longrightarrow Z_{\varepsilon}(s)-z(s)>\eta / 4,
$$

where we have used the fact that $z_{0}-y_{0}(t)=\eta / 2\left(1+e^{A T}\right)$ and the inequalities (7.3) and (7.15). The constants $C(A T)$ and $C_{1}(x, y, A, T)$ in (7.19) must also be chosen sufficiently large. Hence the probability in (7.18) is bounded above by the probability

$$
P\left(\sup _{t \leq s<T}\left|Z_{\varepsilon}(s)-z(s)\right|>\frac{\eta e^{-A T}}{4\left(1+e^{A T}\right)} \mid Z_{\varepsilon}(t)-y_{0}(t)=\frac{\eta}{2\left(1+e^{A T}\right)}\right) .
$$

It is clear from (7.3) that if the constant $C(A T)$ in (7.19) is chosen sufficiently large then we may apply (7.16) to estimate (7.21), since for $C(A T)$ large enough the first inequality in (7.17) is satisfied. Now (7.7) follows from (7.16) since the condition on $\rho$ implies the second inequality in (7.17).

Lemma 7.4. Let $y_{\varepsilon}(s)$ for $0 \leq s<T$, be as in Lemma 7.3 and let $y_{c}(s)$ for $0 \leq s<T$, be the solution to the corresponding classical problem (1.10) which has optimal controller $\lambda_{c}(s)$ for $0 \leq s<T$. Then there is a constant $C(x, y, A, T)$ such that

$E\left\{\int_{0}^{T}\left[\lambda_{\varepsilon}\left(y_{\varepsilon}(s), s\right)-b\left(y_{\varepsilon}(s), s\right)-\lambda_{c}(s)+b\left(y_{c}(s), s\right)\right]^{2} d s\right\} \leq C(x, y, A, T) \varepsilon^{1 / 4}$.

Proof. Following the argument of Lemma 3.5 we define a classical path $y_{\varepsilon, c}(\cdot)$ which corresponds to the stochastic path $y_{\varepsilon}(\cdot)$ by

$$
\frac{d y_{\varepsilon, c}(s)}{d s}=\lambda_{\varepsilon}\left(y_{\varepsilon}(s), s\right)+k / T, \quad 0 \leq s<T
$$


where $y_{\varepsilon, c}(0)=y$ and $k$ is defined by

$$
k=\max \left[x-y-\int_{0}^{T} \lambda_{\varepsilon}\left(y_{\varepsilon}(s), s\right) d s, 0\right] .
$$

Observe from Lemma 4.1 and Theorem 4.4 that the integral on the right-hand side of (7.24) exists with probability 1. Letting $\alpha$ lie in the interval $0<\alpha<1$, and using the fact that $y_{\varepsilon, c}(T) \geq x$, we have that

$$
\begin{aligned}
q(x, y, 0) \leq & \mathcal{F}\left[\alpha y_{\varepsilon, c}(\cdot)+(1-\alpha) y_{c}(\cdot)\right] \\
= & \frac{1}{2} \int_{0}^{T}\left[\alpha\left\{\lambda_{\varepsilon}\left(y_{\varepsilon}(s), s\right)-b\left(y_{\varepsilon}(s), s\right)\right\}\right. \\
& \left.+(1-\alpha)\left\{\lambda_{c}(s)-b\left(y_{c}(s), s\right)\right\}+g_{\varepsilon}(s)-h\left(y_{\varepsilon}(s), s\right)\right]^{2} d s
\end{aligned}
$$

where the deterministic function $h(z, s)$ is given by the formula

$$
h(z, s)=b\left(\alpha z+(1-\alpha) y_{c}(s), s\right)-\alpha b(z, s)-(1-\alpha) b\left(y_{c}(s), s\right),
$$

and the random function $g_{\varepsilon}(s)$ by the formula,

$$
g_{\varepsilon}(s)=\alpha k / T+b\left(\alpha y_{\varepsilon}(s)+(1-\alpha) y_{c}(s), s\right)-b\left(\alpha y_{\varepsilon, c}(s)+(1-\alpha) y_{c}(s), s\right) \text {. }
$$

We expand out $g_{\varepsilon}(\cdot)$ in the quadratic expression in (7.25) to obtain the inequality

$$
\begin{aligned}
& q(x, y, 0) \leq \frac{1}{2} \int_{0}^{T} g_{\varepsilon}(s)^{2} d s+\int_{0}^{T}\left|g_{\varepsilon}(s)\right|\left|h\left(y_{\varepsilon}(s), s\right)\right| d s \\
& +\int_{0}^{T}\left|g_{\varepsilon}(s)\right|\left|\lambda_{c}(s)-b\left(y_{c}(s), s\right)\right| d s+\int_{0}^{T}\left|g_{\varepsilon}(s)\right|\left|\lambda_{\varepsilon}\left(y_{\varepsilon}(s), s\right)-b\left(y_{\varepsilon}(s), s\right)\right| d s \\
& +\frac{1}{2} \int_{0}^{T}\left[\alpha\left\{\lambda_{\varepsilon}\left(y_{\varepsilon}(s), s\right)-b\left(y_{\varepsilon}(s), s\right)\right\}+(1-\alpha)\left\{\lambda_{c}(s)-b\left(y_{c}(s), s\right)\right\}-h\left(y_{\varepsilon}(s), s\right)\right]^{2} d s .
\end{aligned}
$$

Since $b(\cdot, s)$ is concave for $0 \leq s<T$, it follows that the function $h$ is nonnegative. Thus since $\left[\lambda_{\varepsilon}\left(y_{\varepsilon}(s), s\right)-b\left(y_{\varepsilon}(s), s\right)\right]$ and $\left[\lambda_{c}(s)-b\left(y_{c}(s), s\right)\right]$ are both nonnegative, one has the inequality

$$
\begin{gathered}
\frac{1}{2} \int_{0}^{T}\left[\alpha\left\{\lambda_{\varepsilon}\left(y_{\varepsilon}(s), s\right)-b\left(y_{\varepsilon}(s), s\right)\right\}+(1-\alpha)\left\{\lambda_{c}(s)-b\left(y_{c}(s), s\right)\right\}-h\left(y_{\varepsilon}(s), s\right)\right]^{2} d s \\
\leq \frac{\alpha}{2} \int_{0}^{T}\left[\lambda_{\varepsilon}\left(y_{\varepsilon}(s), s\right)-b\left(y_{\varepsilon}(s), s\right)\right]^{2} d s+\frac{1-\alpha}{2} \int_{0}^{T}\left[\lambda_{c}(s)-b\left(y_{c}(s), s\right)\right]^{2} d s \\
\quad-\frac{\alpha(1-\alpha)}{2} \int_{0}^{T}\left[\lambda_{\varepsilon}\left(y_{\varepsilon}(s), s\right)-b\left(y_{\varepsilon}(s), s\right)-\lambda_{c}(s)+b\left(y_{c}(s), s\right)\right]^{2} d s,
\end{gathered}
$$

provided that $h\left(y_{\varepsilon}(s), s\right) \leq 2(1-\alpha)\left[\lambda_{c}(s)-b\left(y_{c}(s), s\right)\right]$ for $0 \leq s<T$. Since we also have that $h(z, s) \leq 2 A \alpha(1-\alpha)\left|z-y_{c}(s)\right|$, we conclude that (7.29) holds provided $y_{\varepsilon}(\cdot)$ satisfies the inequality

$$
A \alpha\left|y_{\varepsilon}(s)-y_{c}(s)\right| \leq\left[\lambda_{c}(s)-b\left(y_{c}(s), s\right)\right], \quad 0 \leq s<T .
$$


If we now use (7.3) and the lower bound in (7.4) we see that (7.30) is implied by the inequality

$$
\left|y_{\varepsilon}(s)-y_{0}(s)\right| \leq\left[\alpha^{-1} C_{1}(A T)-C_{2}(A T)\right] \sqrt{T q(x, y, 0)}, \quad 0 \leq s<T,
$$

for some positive universal constants $C_{1}(A T)$ and $C_{2}(A T)$ depending only on $A T$.

Observe now that from Theorem 1.1 the inequality $(7.22)$ holds if $T q(x, y, 0) \leq$ $\varepsilon^{1 / 4}$, whence we may assume $T q(x, y, 0)>\varepsilon^{1 / 4}$. It follows then from Lemma 7.3 that, for $\alpha$ sufficiently small depending only on $A T$ and $\varepsilon$ sufficiently small depending only on $x, y, A$, and $T$ the inequality (7.31) holds with probability close to 1 .

We now estimate the expectation of the terms in $g_{\varepsilon}(\cdot)$ on the right-hand side of (7.28). From Theorem 4.4 it follows that the quantity $k$ in (7.24) satisfies the inequality

$$
0 \leq k \leq \sqrt{\varepsilon} \max [W(T), 0],
$$

where $W(\cdot)$ is Brownian motion. We also have from (1.14) and (7.23) that

$$
\sup _{0 \leq s<T}\left|y_{\varepsilon}(s)-y_{\varepsilon, c}(s)\right| \leq \sqrt{\varepsilon} \sup _{0 \leq s<T}|W(s)|+k .
$$

We may bound the random function $g_{\varepsilon}(\cdot)$ of $(7.27)$ using (7.32) and (7.33) to obtain

$$
\sup _{0 \leq s<T}\left|g_{\varepsilon}(s)\right| \leq \frac{2 \alpha \sqrt{\varepsilon}}{T}[1+A T] \sup _{0 \leq s<T}|W(s)| .
$$

Evidently (7.34) implies that

$$
E\left[\int_{0}^{T} g_{\varepsilon}(s)^{2} d s\right] \leq \alpha^{2} \varepsilon C_{3}(A T)
$$

for a constant $C_{3}(A T)$ depending only on $A T$. The inequality (7.35) in turn implies by the Schwarz inequality that

$$
E\left[\int_{0}^{T}\left|g_{\varepsilon}(s)\right|\left|\lambda_{c}(s)-b\left(y_{c}(s), s\right)\right| d s\right] \leq \varepsilon^{1 / 2} \alpha C_{4}(A T)[1+q(x, y, 0)]
$$

for a constant $C_{4}(A T)$ depending only on $A T$. Similarly one has by Theorem 1.1 that

$$
\begin{aligned}
& E\left[\int_{0}^{T}\left|g_{\varepsilon}(s)\right|\right.\left.\left|\lambda_{\varepsilon}\left(y_{\varepsilon}(s), s\right)-b\left(y_{\varepsilon}(s), s\right)\right| d s\right] \\
& \leq \sqrt{\varepsilon} \alpha C_{5}(A T)\left[1+q(x, y, 0)+C_{6}(x, y, A, T) \sqrt{\varepsilon}\right]
\end{aligned}
$$

for constants $C_{5}(A T)$ depending only on $A T$ and $C_{6}(x, y, A, T)$ depending on $x, y, A$, and $T$. The final term involving $g_{\varepsilon}(\cdot)$ can be estimated by using Lemma 7.3. Thus

$$
\begin{aligned}
& E\left[\int_{0}^{T}\left|g_{\varepsilon}(s)\right|\left|h\left(y_{\varepsilon}(s), s\right)\right| d s\right] \\
& \quad \leq \alpha \sqrt{\varepsilon} C_{3}(A T)^{1 / 2} 2 A \alpha(1-\alpha) E\left[\int_{0}^{T}\left|y_{\varepsilon}(s)-y_{c}(s)\right|^{2} d s\right]^{1 / 2},
\end{aligned}
$$


and the expectation on the right-hand side of (7.38) is bounded as

$$
E\left[\int_{0}^{T}\left|y_{\varepsilon}(s)-y_{c}(s)\right|^{2} d s\right] \leq C_{7}(A T)\left\{T^{2} q(x, y, 0)+C_{8}(x, y, A, T) \varepsilon^{1 / 2}\right\} .
$$

We now define $p_{\varepsilon}$ as the probability that the inequality (7.31) is violated, and take the expectation of (7.28) over the event (7.31). Thus from (7.28), (7.29), and (7.35)-(7.39) we conclude that

$$
\begin{gathered}
\frac{\alpha(1-\alpha)}{2} E\left[\int_{0}^{T}\left[\lambda_{\varepsilon}\left(y_{\varepsilon}(s), s\right)-b\left(y_{\varepsilon}(s), s\right)-\lambda_{c}(s)+b\left(y_{c}(s), s\right)\right]^{2} d s ;(7.31) \text { holds }\right] \\
\leq\left[p_{\varepsilon}+\varepsilon^{1 / 2} \alpha C_{9}(A T)\right] q(x, y, 0)+\alpha C_{10}(x, y, A, T) \varepsilon^{1 / 4} .
\end{gathered}
$$

Since we can estimate $p_{\varepsilon}$ from Lemma 7.3, we can conclude (7.22) from (7.40) provided we can estimate the expectation

$$
E\left[\int_{0}^{T}\left[\lambda_{\varepsilon}\left(y_{\varepsilon}(s), s\right)-b\left(y_{\varepsilon}(s), s\right)\right]^{2} d s ;(7.31) \text { does not hold }\right]
$$

appropriately. We have now from Corollary 6.3 that

$$
\begin{aligned}
E\left[\int_{0}^{T-\delta}\left[\lambda_{\varepsilon}\left(y_{\varepsilon}(s), s\right)-b\left(y_{\varepsilon}(s), s\right)\right]^{2} d s ;(7.31) \text { does not hold }\right] \\
\leq 2 p_{\varepsilon}^{1 / 2}(1+A T)^{2} \int_{0}^{T-\delta} \frac{d s}{T-s} E\left[q_{\varepsilon}\left(x, y_{\varepsilon}(s), s\right)^{2}\right]^{1 / 2}
\end{aligned}
$$

Let $Y_{\varepsilon}(s)$ for $s \geq 0$, be the solution to (1.5) with $Y_{\varepsilon}(0)=y$. Recall that since $\partial q_{\varepsilon}(x, z, s) / \partial z \leq 0$ we have that $y_{\varepsilon}(s) \geq Y_{\varepsilon}(s)$ for $s \geq 0$. Using Lemma 3.1 then, we conclude that

$$
E\left[q_{\varepsilon}\left(x, y_{\varepsilon}(s), s\right)^{2}\right] \leq \frac{1}{(T-s)^{2}}\left[C_{3}(A T) E\left[\left\{x-Y_{\varepsilon}(s)\right\}^{4}\right]+C_{4}(x, y, A, T)\right]
$$

We are left now to estimate

$$
E\left[\int_{T-\delta}^{T}\left[\lambda_{\varepsilon}\left(y_{\varepsilon}(s), s\right)-b\left(y_{\varepsilon}(s), s\right)\right]^{2} d s ;(7.31) \text { does not hold }\right]
$$

for some $\delta>0$. Instead of attempting to show that the expectation (7.44) is small, we consider, as for (7.29), under what circumstances the inequality

$$
\begin{aligned}
{\left[\alpha \left\{\lambda_{\varepsilon}\left(y_{\varepsilon}(s), s\right)\right.\right.} & \left.\left.-b\left(y_{\varepsilon}(s), s\right)\right\}+(1-\alpha)\left\{\lambda_{c}(s)-b\left(y_{c}(s), s\right)\right\}-h\left(y_{\varepsilon}(s), s\right)\right]^{2} \\
\leq & \frac{\alpha}{2}\left[\lambda_{\varepsilon}\left(y_{\varepsilon}(s), s\right)-b\left(y_{\varepsilon}(s), s\right)\right]^{2}+\frac{(1-\alpha)}{2}\left[\lambda_{c}(s)-b\left(y_{c}(s), s\right)\right]^{2} \\
& -\frac{\alpha(1-\alpha)}{2}\left[\lambda_{\varepsilon}\left(y_{\varepsilon}(s), s\right)-b\left(y_{\varepsilon}(s), s\right)-\lambda_{c}(s)+b\left(y_{c}(s), s\right)\right]^{2}
\end{aligned}
$$


holds if $s$ lies in the interval $T-\delta<s<T$. From Lemma 6.1 we see that if $\delta>0$ is sufficiently small and depends only on $A$, then (7.45) holds if $y_{\varepsilon}(s)$ satisfies the one-sided inequality

$$
y_{\varepsilon}(s)-y_{0}(s) \leq\left[\alpha^{-1} C_{1}(A T)-C_{2}(A T)\right] \sqrt{T q(x, y, 0)}
$$

similar to (7.31). Thus, instead of estimating (7.44), it will be sufficient to estimate

$$
E\left[\int_{T-\delta}^{T}\left[\lambda_{\varepsilon}\left(y_{\varepsilon}(s), s\right)-b\left(y_{\varepsilon}(s), s\right)\right]^{2} \chi\left(y_{\varepsilon}(s), s\right) d s\right],
$$

where

$$
\begin{aligned}
& \chi(z, s)=1 \quad \text { if } z>y_{0}(s)+\left[\alpha^{-1} C_{1}(A T)-C_{2}(A T)\right] \sqrt{T q(x, y, 0)}, \\
& \chi(z, s)=0, \quad \text { otherwise. }
\end{aligned}
$$

Using (7.8) and (7.9) we see that if $\chi\left(y_{\varepsilon}(s), s\right)=1$ then $\left[\lambda_{\varepsilon}\left(y_{\varepsilon}(s), s\right)-b\left(y_{\varepsilon}(s), s\right)\right]^{2}$ is bounded, whence we conclude that the expectation $(7.47)$ is bounded by a constant $C(x, y, A, T)$ depending only on $x, y, A$, and $T$. The result follows from Lemma 7.3.

Proof of Theorem 1.3. We use the representation for $\partial q(x, y, t) / \partial y$ given by $(2.29)$ and for $\partial q_{\varepsilon}(x, y, t) / \partial y$ by (6.7). Thus we have that

$$
\begin{aligned}
& \frac{\partial q}{\partial y}(x, y, 0)-\frac{\partial q_{\varepsilon}}{\partial y}(x, y, 0)=\frac{1}{T} E\left\{\int_{0}^{T}\left[1+(T-s) \frac{\partial b}{\partial y}\left(y_{\varepsilon}(s), s\right)\right]\right. \\
& \left.\cdot\left[\lambda_{\varepsilon}\left(y_{\varepsilon}(s), s\right)-b\left(y_{\varepsilon}(s), s\right)-\lambda_{c}(s)+b\left(y_{c}(s), s\right)\right] d s\right\} \\
& +\frac{1}{T} E\left\{\int_{0}^{T}(T-s)\left[\frac{\partial b}{\partial y}\left(y_{\varepsilon}(s), s\right)-\frac{\partial b}{\partial y}\left(y_{c}(s), s\right)\right]\left[\lambda_{c}(s)-b\left(y_{c}(s), s\right)\right] d s\right\}
\end{aligned}
$$

In view of Lemma 7.4 the second identity of (1.25) follows if we can show that

$$
\lim _{\varepsilon \rightarrow 0} E\left\{\int_{0}^{T}\left|\frac{\partial b}{\partial y}\left(y_{\varepsilon}(s), s\right)-\frac{\partial b}{\partial y}\left(y_{c}(s), s\right)\right| d s\right\}=0 .
$$

We put $\phi_{\varepsilon}(s)=y_{\varepsilon}(s)-y_{c}(s)$ for $0 \leq s<T$, and observe that $\phi_{\varepsilon}(s)$ satisfies the equation

$$
d \phi_{\varepsilon}(s)=\left[\lambda_{\varepsilon}\left(y_{\varepsilon}(s), s\right)-\lambda_{c}(s)\right] d s+\sqrt{\varepsilon} d W(s), \quad \phi_{\varepsilon}(0)=0 .
$$

It follows from (7.51) that, for $0 \leq t<T$,

$$
\begin{aligned}
\left|\phi_{\varepsilon}(t)\right| \leq & \int_{0}^{t}\left|\lambda_{\varepsilon}\left(y_{\varepsilon}(s), s\right)-b\left(y_{\varepsilon}(s), s\right)-\lambda_{c}(s)+b\left(y_{c}(s), s\right)\right| d s \\
& +A \int_{0}^{t}\left|\phi_{\varepsilon}(s)\right| d s+\sqrt{\varepsilon}|W(t)| .
\end{aligned}
$$

Using Gronwall's inequality in (7.52) and Lemma 7.4 we see that (7.50) holds. This proves the second identity of (1.25). The first identity follows in a similar way. 


\section{A. Log concavity of solutions to linear diffusion equations}

Our goal in this appendix is to establish convexity properties of the function $q_{\varepsilon}(x, y, t)$ defined by (1.7). We shall first show convexity in $y$ for fixed $x \in \mathbb{R}$ and $t<T$, since showing joint convexity in $(x, y)$ is considerably more difficult. We consider the terminal boundary value problem

$$
\begin{cases}\frac{\partial w}{\partial t}+b(y, t) \frac{\partial w}{\partial y}+\frac{\varepsilon}{2} \frac{\partial^{2} w}{\partial y^{2}}=0, & y>0, t<T, \\ w(y, T)=w_{0}(y), & y>0 ; w(0, t)=0, t<T .\end{cases}
$$

Proposition A.1. Assume $b(\cdot, \cdot)$ satisfies (1.1) and the terminal function $w_{0}(y)$ is $C^{2}$ for $y>0$ and $C^{1}$ for $y \geq 0$ with $w_{0}(0)=0$. Assume further that

$$
\sup _{y>0}\left\{\left|w_{0}(y)\right|+\left|d w_{0}(y) / d y\right|+\left|d^{2} w_{0}(y) / d y^{2}\right|\right\}<\infty \text {. }
$$

Then there is a unique solution $w(y, t)$ for $y>0$ and $t<T$, to the terminal boundary value problem (A.1) which has the property that $w(y, t)$ is $C^{2}$ in $y, C^{1}$ in $t$, and satisfies the inequality

$$
\sup _{y>0, T_{0}<t<T}\left\{|w(y, t)|+|\partial w(y, t) / \partial y|+\left|\partial^{2} w(y, t) / \partial y^{2}\right|\right\}<\infty
$$

for any $T_{0}<T$. In addition, the functions $w(y, t)$ and $\partial w(y, t) / \partial y$ are continuous for $y \geq 0$ and $t \leq T$.

Proof. We first observe that the result holds when $b \equiv 0$. In this case the solution is given by the method of images as

$$
w(y, t)=\int_{0}^{\infty}\left[G\left(y-y^{\prime}, \varepsilon(T-t)\right)-G\left(y+y^{\prime}, \varepsilon(T-t)\right)\right] w_{0}\left(y^{\prime}\right) d y^{\prime},
$$

where $G(\cdot, s)$ is the probability density function of the Gaussian variable with mean 0 and variance $s$. Thus on using integration by parts we have

$$
\frac{\partial w}{\partial y}(y, t)=\int_{0}^{\infty}\left[G\left(y-y^{\prime}, \varepsilon(T-t)\right)+G\left(y+y^{\prime}, \varepsilon(T-t)\right)\right] \frac{d w_{0}\left(y^{\prime}\right)}{d y^{\prime}} d y^{\prime},
$$

where we have used the fact that $w_{0}(0)=0$ in deriving (A.5).

Upon a further integration by parts we have that

$$
\frac{\partial^{2} w}{\partial y^{2}}(y, t)=\int_{0}^{\infty}\left[G\left(y-y^{\prime}, \varepsilon(T-t)\right)-G\left(y+y^{\prime}, \varepsilon(T-t)\right)\right] \frac{d^{2} w_{0}\left(y^{\prime}\right)}{d y^{\prime 2}} d y^{\prime}
$$

It follows easily from (A.4)-(A.6) that (A.3) holds. In addition $w(y, t)$ and $\partial w(y, t) / \partial y$ are continuous for $y \geq 0$ and $t \leq T$. We also have that $\partial^{2} w(y, t) / \partial y^{2}$ is continuous for $y>0$ and $t \leq T$, provided $d^{2} w_{0}(y) / d y^{2}$ is continuous in $y>0$.

To prove the result for general $b(\cdot, \cdot)$ satisfying $(1.1)$ it will be sufficient to establish it for $t$ restricted to a small interval $[T-\Delta, T]$. We proceed as in Lemma 3.4. 
Taking $y_{1}=\eta$ in (3.18) we see from (3.19) that $w(y, t)$ is given by the formula

$$
w(y, t)=\int_{0}^{2 \eta} G\left(y, y^{\prime}, t, T\right) w_{0}\left(y^{\prime}\right) d y^{\prime}-\varepsilon \int_{t}^{T} d s w_{+}(s) \frac{\partial G}{\partial y^{\prime}}(y, 2 \eta, t, s),
$$

provided $0<y<2 \eta$. The Green's function $G\left(y, y^{\prime}, t, T\right)$ is defined by the perturbation expansion (3.23). Since $w_{+}(\cdot)$ is bounded by virtue of (A.2), we see that if $\Delta$ satisfies (3.20) then $\sup \{|w(y, t)|: 0<y \leq \eta, T-\Delta \leq t<T\}<\infty$ and $w(y, t)$ is continuous for $0 \leq y \leq \eta$ and $T-\Delta \leq t \leq T$, with $w(0, t)=0$.

We consider next the first derivative $\partial w(y, t) / \partial y$, which from (A.7) is given by the formula

(A.8) $\frac{\partial w}{\partial y}(y, t)=\int_{0}^{2 \eta} \frac{\partial G}{\partial y}\left(y, y^{\prime}, t, T\right) w_{0}\left(y^{\prime}\right) d y^{\prime}-\varepsilon \int_{t}^{T} d s w_{+}(s) \frac{\partial^{2} G}{\partial y \partial y^{\prime}}(y, 2 \eta, t, s)$.

It is evident from (3.41) that the second integral on the right-hand side of (A.8) is uniformly bounded in the set $\{(y, t): 0<y \leq \eta, T-\Delta \leq t<T\}$ and that the integral converges to 0 as $t \rightarrow T$, uniformly for $0<y \leq \eta$. To estimate the first integral on the right-hand side of (A.8) we integrate by parts the first term in the perturbation expansion (3.23) for $G\left(y, y^{\prime}, t, T\right)$. Just as in (A.5) we see that this term is uniformly bounded in the set $\{(y, t): 0<y \leq \eta, T-\Delta \leq t<T\}$, and converges uniformly to $d w_{0}(y) / d y$ as $t \rightarrow T$. We can estimate the higher order terms

$$
\int_{0}^{2 \eta} \frac{\partial v_{n}}{\partial y}\left(y, y^{\prime}, t, T\right) w_{0}\left(y^{\prime}\right) d y^{\prime}
$$

for $n \geq 0$ simply by using (3.24). Thus we see that the sum of the higher order terms is uniformly bounded in the set $\{(y, t): 0<y \leq \eta, T-\Delta \leq t<T\}$. To prove continuity of $\partial w(y, t) / \partial y$ as $t \rightarrow T$ we need to show that the integral in (A.9) converges uniformly to 0 as $t \rightarrow T$ in the interval $0<y \leq \eta$. This follows from (3.24) when $n \geq 1$. To prove it for $n=0$ we again need to make use of integration by parts. Thus we see that

$$
\begin{aligned}
& \left|\int_{0}^{2 \eta} g_{0}\left(z, y^{\prime}, s, T\right) w_{0}\left(y^{\prime}\right) d y^{\prime}\right| \\
& 0) \leq C|b(z, s)|\left[\sup _{0<y \leq 2 \eta}\left|d w_{0}(y) / d y\right|+G(z-2 \eta, 2 \varepsilon(T-s))\left|w_{0}(2 \eta)\right|\right],
\end{aligned}
$$

for some universal constant $C$.

It follows from (A.10) and the representation (3.23) for $v_{0}$ that the integral (A.9) also converges to 0 as $t \rightarrow T$ when $n=0$. We have shown that $\sup \{|\partial w(y, t) / \partial y|$ : $0<y \leq \eta, T-\Delta \leq t<T\}<\infty$ and $\partial w(y, t) / \partial y$ is continuous for $0 \leq y \leq \eta$ and $T-\Delta \leq t \leq T$.

To estimate the second derivative $\partial^{2} w(y, t) / \partial y^{2}$ we proceed in a similar manner:

$$
\text { (A.11) } \frac{\partial^{2} w}{\partial y^{2}}(y, t)=\int_{0}^{2 \eta} \frac{\partial^{2} G}{\partial y^{2}}\left(y, y^{\prime}, t, T\right) w_{0}\left(y^{\prime}\right) d y^{\prime}-\varepsilon \int_{t}^{T} d s w_{+}(s) \frac{\partial^{3} G}{\partial y^{2} \partial y^{\prime}}(y, 2 \eta, t, s) \text {. }
$$


We wish to show that $\sup \left\{\left|\partial^{2} w(y, t) / \partial y^{2}\right|: 0<y \leq \eta, T-\Delta \leq t<T\right\}<\infty$. In view of (3.46)) it is sufficient to consider only the first integral on the right-hand side of (A.11). We estimate the first term in the perturbation expansion (3.23) for $G\left(y, y^{\prime}, t, T\right)$ using integration by parts as in (A.6). The higher order terms, corresponding to $v_{n}\left(y, y^{\prime}, t, T\right)$ with $n \geq 1$, can be estimated using (3.39), so we are only left to deal with the term corresponding to $v_{0}\left(y, y^{\prime}, t, T\right)$. We can estimate this by using (A.10)) and the corresponding inequality for the derivative of $g_{0}$ :

$$
\begin{aligned}
& \text { (A.12) }\left|\int_{0}^{2 \eta} \frac{\partial g_{0}}{\partial z}\left(z, y^{\prime}, s, T\right) w_{0}\left(y^{\prime}\right) d y^{\prime}\right| \\
& \leq C\left[A+\left\{\frac{\nu}{\Delta(T-t)}\right\}^{1 / 2}\right]\left[\sup _{0<y \leq 2 \eta}\left|d w_{0}(y) / d y\right|+G(z-2 \eta, 2 \varepsilon(T-s))\left|w_{0}(2 \eta)\right|\right]
\end{aligned}
$$

for some universal constant $C$. We have shown that $\sup \left\{\left|\partial^{2} w(y, t) / \partial y^{2}\right|: 0<y \leq\right.$ $\eta, T-\Delta \leq t<T\}<\infty$.

We can easily extend the estimates we have made on $w(y, t)$ and its $y$ derivatives in the set $\{(y, t): 0<y \leq \eta, T-\Delta \leq t<T\}$ to all of $y>0$ by observing that the function $v(z, t)$ defined by $v(z, t)=w(z+y(t), t)$, where $y(s)$ for $s \leq T$, is a solution to (1.11) with $y(T)=y_{1}$, satisfies the PDE

$$
\frac{\partial v}{\partial t}+[b(z+y(t), t)-b(y(t), t)] \frac{\partial v}{\partial z}+\frac{\varepsilon}{2} \frac{\partial^{2} v}{\partial z^{2}}=0 .
$$

Then we represent $v(z, t)$ by a formula similar to (A.7) and use perturbation theory as before, observing that the perturbation series for the Green's function converges in a region $\{|z|<\eta, T-\Delta \leq t<T\}$, where $\eta$ and $\Delta$ can be taken to be independent of $y_{1}$.

Theorem A.2. Suppose $b(\cdot, \cdot)$ satisfies (1.1), and in addition the function $b(y, t)$ is concave in $y$ for $y \in \mathbb{R}$ and $t \leq T$. Then for any fixed $x \in \mathbb{R}$ and $0 \leq t<T$, the function $q_{\varepsilon}(x, y, t)$ of $(1.7)$ is a convex function of $y \in \mathbb{R}$.

Proof. We shall take without loss of generality $x=0$. For $\delta$ satisfying $0<\delta<1$ we define a function $g_{\delta}(z)$ with domain $\{z \in \mathbb{R}: z>-1\}$ by

$$
\begin{array}{lll}
g_{\delta}^{\prime \prime}(z)=1 /(1+z)^{2}, & \text { if }-1<z<-(1-\delta), \\
g_{\delta}^{\prime \prime}(z)=-z / \delta^{2}(1-\delta) & \text { if } \quad-(1-\delta)<z<0, \\
g_{\delta}(0)=g_{\delta}^{\prime}(0)=0, & & \\
g_{\delta}^{\prime \prime}(z)=0, & \text { if } z>0 .
\end{array}
$$

Evidently $g_{\delta}(z)$ is a $C^{2}$ convex decreasing function which has the property that $g_{\delta}(z)=0$ for $z>0$ and $g_{\delta}(z) \sim K_{\delta}-\log (1+z)$ as $z \rightarrow-1$, where $K_{\delta}$ is a constant depending on $\delta$. For $\Lambda>0$ and $y>-\Lambda$ let $\tau_{\Lambda, y, t}$ be the first hitting time at $-\Lambda$ for the diffusion $Y_{\varepsilon}(s)$ with $s \geq t$, of (1.5) with $Y_{\varepsilon}(t)=y$. We define a function $u_{\varepsilon, \Lambda, \delta}(y, t)$ by

$$
u_{\varepsilon, \Lambda, \delta}(y, t)=E\left\{\exp \left[-g_{\delta}\left(Y_{\varepsilon}(T) / \Lambda\right)\right] ; \tau_{\Lambda, y, t}>T\right\}
$$


Letting $\delta \rightarrow 0$ in (A.15) we conclude from (A.14) that

$$
P\left(Y_{\varepsilon}(T)>0 ; \tau_{\Lambda, y, t}>T \mid Y_{\varepsilon}(t)=y\right)=\lim _{\delta \rightarrow 0} u_{\varepsilon, \Lambda, \delta}(y, t) .
$$

It is also clear from (1.6) that

$$
u_{\varepsilon}(0, y, t)=\lim _{\Lambda \rightarrow \infty} P\left(Y_{\varepsilon}(T)>0 ; \tau_{\Lambda, y, t}>T \mid Y_{\varepsilon}(t)=y\right) .
$$

We conclude from (A.16) and (A.17) that the convexity of $q_{\varepsilon}(0, y, t)$ in $y$ follows from the logarithmic concavity of the function $u_{\varepsilon, \Lambda, \delta}(y, t)$ in $y$.

To prove logarithmic concavity we first observe that $u_{\varepsilon, \Lambda, \delta}(y, t)$ satisfies the $\operatorname{PDE}(1.2)$ for $y>-\Lambda$ and $t<T$, with Dirichlet boundary condition $u_{\varepsilon, \Lambda, \delta}(y, t)=0$ at $y=-\Lambda$, and terminal data

$$
u_{\varepsilon, \Lambda, \delta}(y, T)=\exp \left[-g_{\delta}(y / \Lambda)\right], \quad y>-\Lambda .
$$

Since the function (A.18) is increasing in $y$, it follows from the maximum principle that for $t<T$ the function $u_{\varepsilon, \Lambda, \delta}(y, t)$ is also an increasing function of $y$. From (A.14) we see that $u_{\varepsilon, \Lambda, \delta}(y, T)$ is $C^{2}$ for $y \geq-\Lambda$, and $u_{\varepsilon, \Lambda, \delta}(-\Lambda, T)=0$ and $\partial u_{\varepsilon, \Lambda, \delta}(-\Lambda, T) / \partial y>0$. Therefore we can apply the regularity result of Proposition A.1. It follows from this and the Hopf maximum principle [19] that

$$
\partial u_{\varepsilon, \Lambda, \delta}(-\Lambda, t) / \partial y>0, \quad t \leq T .
$$

Next as in (1.7) we put $u_{\varepsilon, \Lambda, \delta}(y, t)=\exp \left[-q_{\varepsilon, \Lambda, \delta}(y, t) / \varepsilon\right]$, and observe that $q_{\varepsilon, \Lambda, \delta}(y, t)$ satisfies the PDE (1.8). Since $u_{\varepsilon, \Lambda, \delta}(y, t)$ is an increasing function of $y$, it follows that $q_{\varepsilon, \Lambda, \delta}(y, t)$ is a decreasing function of $y$. Hence $q_{\varepsilon, \Lambda, \delta}(y, t)$ is a solution to the PDE

$$
\frac{\partial q_{\varepsilon, \Lambda, \delta}}{\partial t}+\frac{\varepsilon}{2} \frac{\partial^{2} q_{\varepsilon, \Lambda, \delta}}{\partial y^{2}}-B\left(y, t, \frac{\partial q_{\varepsilon, \Lambda, \delta}}{\partial y}\right)=0,
$$

where the function $B(y, t, p)$ is defined by

$$
B(y, t, p)=b(y, t)|p|+p^{2} / 2 .
$$

Observe that the function $B(y, t, p)$ is concave in $y$ for all $p \in \mathbb{R}$ and $t \leq T$. Applying Theorem 4.1 of [9] to (A.20) we see that $q_{\varepsilon, \Lambda, \delta}(y, t)$ is convex in $y$ for $y>-\Lambda$ and $t<T$, provided we can show that the expression

$$
q_{\varepsilon, \Lambda, \delta}\left(\left(y+y^{\prime}\right) / 2, t\right)-\left[q_{\varepsilon, \Lambda, \delta}(y, t)+q_{\varepsilon, \Lambda, \delta}\left(y^{\prime}, t\right)\right] / 2
$$

is less than or equal to 0 as $\left(y, y^{\prime}, t\right)$ approaches $\left(y_{\infty}, y_{\infty}^{\prime}, t_{\infty}\right)$ with $t_{\infty} \leq T$ finite, and $\left(y_{\infty}, y_{\infty}^{\prime}\right)$ on the boundary of $(-\Lambda, \infty)^{2} \subset \mathbb{R}^{2}$ if $t_{\infty}<T$, and an arbitrary point in the closure of $(-\Lambda, \infty)^{2}$ if $t_{\infty}=T$.

Suppose now that $y_{\infty}=-\Lambda$ and $-\Lambda<y_{\infty}^{\prime} \leq \infty$. From (A.15) we see that $u_{\varepsilon, \Lambda, \delta}(y, t)>0$ for $y>-\Lambda$ and $t \leq T$, whence the limits of the first and third terms in (A.22) are finite as $\left(y, y^{\prime}, t\right) \rightarrow\left(y_{\infty}, y_{\infty}^{\prime}, t_{\infty}\right)$, whereas the second term 
converges to $-\infty$. Thus we may assume $y_{\infty}^{\prime}=y_{\infty}=-\Lambda$. In that case we observe that the exponential of $\varepsilon^{-1}$ times the expression (A.22) is the same as

$$
\left[u_{\varepsilon, \Lambda, \delta}(y, t) u_{\varepsilon, \Lambda, \delta}\left(y^{\prime}, t\right)\right]^{1 / 2} / u_{\varepsilon, \Lambda, \delta}\left(\left\{y+y^{\prime}\right\} / 2, t\right) .
$$

From (A.19) we may write (A.23) as

$$
p\left(z, z^{\prime}, t\right)\left(z z^{\prime}\right)^{1 / 2} /\left[\left(z+z^{\prime}\right) / 2\right],
$$

where $z=y+\Lambda, z^{\prime}=y^{\prime}+\Lambda$, and $\lim \left\{p\left(z, z^{\prime}, t\right): z, z^{\prime} \rightarrow 0, t \rightarrow t_{\infty}\right\}=1$. Thus since the arithmetic mean exceeds the geometric mean, it follows from (A.24) that the limit of $(\mathrm{A} .22)$ as $\left(y, y^{\prime}, t\right) \rightarrow\left(-\Lambda,-\Lambda, t_{\infty}\right)$ is less than or equal to 0 .

For $t_{\infty}=T$, the nonpositivity of $(\mathrm{A} .22)$ for any $\left(y_{\infty}, y_{\infty}^{\prime}\right)$ in the closure of $(-\Lambda, \infty)^{2}$ follows from Proposition A.1 and the convexity of $g_{\delta}(\cdot)$.

Theorem A.3. Suppose $b(\cdot, \cdot)$ satisfies (1.1). Then $\partial^{2} q_{\varepsilon}(x, y, t) / \partial x \partial y \leq 0$ for $x, y \in \mathbb{R}$ and $0 \leq t<T$.

Proof. It will be sufficient to show that for any $h>0$ the function $q_{\varepsilon}(x+h, y, t)-$ $q_{\varepsilon}(x, y, t)$ is a decreasing function of $y$. Letting $g(z)$ be the function

$$
g(z)=z^{2}, \quad z<0 ; \quad g(z)=0, \quad z \geq 0,
$$

we define $u_{\varepsilon, \delta}(x, y, t)$ similarly to (A.15) by

$$
u_{\varepsilon, \delta}(x, y, t)=E\left\{\exp \left[-g\left(\frac{Y_{\varepsilon}(T)-x}{\delta}\right)\right] \mid Y_{\varepsilon}(t)=y\right\} .
$$

Evidently $\lim _{\delta \rightarrow 0} u_{\varepsilon, \delta}(x, y, t)=u_{\varepsilon}(x, y, t)$ and hence the function $q_{\varepsilon, \delta}(x, y, t)=$ $-\varepsilon \log u_{\varepsilon, \delta}(x, y, t)$ satisfies $\lim _{\delta \rightarrow 0} q_{\varepsilon, \delta}(x, y, t)=q_{\varepsilon}(x, y, t)$. Arguing as in the proof of Lemma 3.1, we also see that $q_{\varepsilon, \delta}(x, y, t)$ satisfies the inequality

$$
0 \leq q_{\varepsilon, \delta}(x, y, t) \leq C\left[(x-y)^{2} H(x-y)+1\right], \quad y \in \mathbb{R}, 0 \leq t<T,
$$

where $H(\cdot)$ is the Heaviside function and $C$ is a constant.

In order to prove that $q_{\varepsilon}(x+h, y, t)-q_{\varepsilon}(x, y, t)$ is decreasing in $y$ it will be sufficient to show that the function $v_{\varepsilon, \delta}(y, t)=q_{\varepsilon, \delta}(x+h, y, t)-q_{\varepsilon, \delta}(x, y, t)$ is decreasing in $y$ for any $\delta>0$. To see this we note that $v_{\varepsilon, \delta}$ satisfies a PDE

$$
\frac{\partial v_{\varepsilon, \delta}}{\partial t}+b_{\varepsilon, \delta}(y, t) \frac{\partial v_{\varepsilon, \delta}}{\partial y}+\frac{\varepsilon}{2} \frac{\partial^{2} v_{\varepsilon, \delta}}{\partial y^{2}}=0, \quad y \in \mathbb{R}, t<T,
$$

where the $\operatorname{drift} b_{\varepsilon, \delta}(\cdot, \cdot)$ is given by the formula

$$
b_{\varepsilon, \delta}(y, t)=b(y, t)-\frac{1}{2} \frac{\partial q_{\varepsilon, \delta}(x+h, y, t)}{\partial y}-\frac{1}{2} \frac{\partial q_{\varepsilon, \delta}(x, y, t)}{\partial y} .
$$

The terminal data for $v_{\varepsilon, \delta}$ is given by

$$
v_{\varepsilon, \delta}(y, T)= \begin{cases}h[2(x-y)+h] / \delta^{2}, & \text { if } y<x, \\ {[x+h-y]^{2} / \delta^{2},} & \text { if } x<y<x+h, \\ 0, & \text { if } y>x+h .\end{cases}
$$


Consider now the diffusion process $Y_{\varepsilon, \delta}(s)$ defined by

$$
d Y_{\varepsilon, \delta}(s)=b_{\varepsilon, \delta}\left(Y_{\varepsilon, \delta}(s), s\right) d s+\sqrt{\varepsilon} d W(s) .
$$

From Lemma 3.4 we see that the drift $b_{\varepsilon, \delta}(y, s)$ is uniformly Lipschitz in $y$ in any region $y \geq y_{0}, 0 \leq t \leq T-\eta$, where $y_{0} \in \mathbb{R}$ and $\eta>0$ can be arbitrary. Let $\tau_{y, t}$ be the first hitting time at $y_{0}$ for $Y_{\varepsilon, \delta}(\cdot)$ with $Y_{\varepsilon, \delta}(t)=y>y_{0}$. Then we have the representation

$$
\begin{aligned}
v_{\varepsilon, \delta}(y, t)= & E\left[v_{\varepsilon, \delta}\left(Y_{\varepsilon, \delta}(T-\eta), T-\eta\right) ; \tau_{y, t}>T-\eta\right] \\
& +E\left[v_{\varepsilon, \delta}\left(Y_{\varepsilon, \delta}\left(\tau_{y, t}\right), \tau_{y, t}\right) ; \tau_{y, t}<T-\eta\right] .
\end{aligned}
$$

Observe that from (A.29) we have that $b_{\varepsilon, \delta}(y, s) \geq b(y, s)$ for $t \leq s<T$. Hence using (A.27) we may take the limit $y_{0} \rightarrow-\infty$ in (A.32) to conclude that

$$
v_{\varepsilon, \delta}(y, t)=E\left[v_{\varepsilon, \delta}\left(Y_{\varepsilon, \delta}(T-\eta), T-\eta\right) \mid Y_{\varepsilon, \delta}(t)=y\right] .
$$

If $v_{\varepsilon, \delta}(z, T-\eta)$ were known to be a decreasing function of $z$ then it would follow from (A.33) that $v_{\varepsilon, \delta}(y, t)$ is a decreasing function of $y$. Since $u_{\varepsilon, \delta}(x, y, T-\eta)$ converges uniformly on any finite interval $a \leq y \leq b$ as $\eta \rightarrow 0$ to the function $\exp [-g(y-x) / \delta]$, we see that $v_{\varepsilon, \delta}(z, T-\eta)$ converges uniformly on any finite interval as $\eta \rightarrow 0$ to the decreasing function (A.30). Thus we can still conclude from (A.33) that $v_{\varepsilon, \delta}(y, t)$ is a decreasing function of $y$. The result follows.

It appears that one cannot prove the convexity of $q_{\varepsilon}(x, y, t)$ as a function of $x$ for fixed $y$ directly, in analogy to Theorem A.2, so we shall proceed to showing that $q_{\varepsilon}(x, y, t)$ is convex jointly in $(x, y)$. To do this we consider solutions $v(x, y, t)$ to the semi-linear equation

$$
\frac{\partial v}{\partial t}+b(y, t)\left|\frac{\partial v}{\partial y}\right|+\frac{\varepsilon}{2} \frac{\partial^{2} v}{\partial y^{2}}+\frac{\varepsilon^{\prime}}{2} \frac{\partial^{2} v}{\partial x^{2}}=0, \quad t<T,
$$

in the disk $D_{R}=\left\{(x, y): x^{2}+y^{2}<R^{2}\right\}$, with Dirichlet boundary condition and given terminal data. Thus we wish to solve (A.34) subject to the conditions

$$
v(x, y, T)=v_{0}(x, y),(x, y) \in D_{R} ; \quad v(x, y, t)=0,(x, y) \in \partial D_{R}, t<T .
$$

Using classical techniques [7], [15] for proving regularity of solutions to semilinear parabolic equations, we can establish the following result:

Proposition A.4. Assume $b(\cdot, \cdot)$ satisfies (1.1) and the terminal function $v_{0}(x, y)$ is $C^{2}$ for $(x, y)$ in the closure $\bar{D}_{R}$ of $D_{R}$, with $v_{0}(x, y)=0$ for $(x, y) \in \partial D_{R}$. Then there is a unique solution $v(x, y, t)$ for $(x, y) \in D_{R}$ and $t<T$, to the terminal value problem (A.34) and (A.35), which has the property that $v(x, y, t)$ is $C^{2}$ in $(x, y), C^{1}$ in $t$, and satisfies the inequality

$$
\sup _{T_{0}<t<T}\left\{|v(x, y, t)|+|D v(x, y, t)|+\left|D^{2} v(x, y, t)\right|:(x, y) \in D_{R}\right\}<\infty
$$


for any $T_{0}<T$. In (A.36) $D v(x, y, t)$ denotes the gradient of $v(x, y, t)$ with respect to $(x, y)$, and $D^{2} v(x, y, t)$ the Hessian with respect to $(x, y)$. Additionally, the functions $v(x, y, t)$ and $D v(x, y, t)$ are continuous for $(x, y) \in \bar{D}_{R}$ and $t \leq T$. The tangential second derivative $(y \partial / \partial x-x \partial / \partial y) D v(x, y, t)$ is also continuous.

Next we need to establish a Hopf maximum principle (A.19) for solutions to (A.34) and (A.35).

Lemma A.5. Suppose $v_{0}(x, y)$, defined for $(x, y) \in \bar{D}_{R}$, satisfies the conditions of Proposition A.4, and in addition $0 \leq v_{0}(x, y) \leq 1$ and $(x, y) \in \bar{D}_{R}$. Then if $v_{0} \not \equiv 0$, the solution $v(x, y, t)$ of (A.34) and (A.35) satisfies the inequalities

$$
\begin{aligned}
& 0<v(x, y, t)<1, \quad(x, y) \in D_{R}, \quad t<T, \\
& x \frac{\partial v}{\partial x}(x, y, t)+y \frac{\partial v}{\partial y}(x, y, t)<0, \quad(x, y) \in \partial D_{R}, \quad t<T .
\end{aligned}
$$

Proof. The fact that $0 \leq v(x, y, t) \leq 1$ for $(x, y) \in D_{R}$ and $t<T$, follows by applying the argument for the weak maximum principle, Theorem 1 of Chapter 3 of [19], to the quasilinear equation (A.34). Similarly one sees that the argument for the strong maximum principle, Theorem 2 of Chapter 3 in [19], applies to (A.34). We conclude that (A.37) holds. Finally (A.38) follows by applying the argument of Theorem 3 of Chapter 3 in [19] to (A.34).

The final result we need in order to apply the method of Korevaar [14] to prove convexity in $(x, y)$ of $q_{\varepsilon}(x, y, t)$ is in effect a comparison principle for solutions of the quasilinear equation (A.34) to solutions of the linear equation

$$
\frac{\partial v}{\partial t}+b(y, t) \frac{\partial v}{\partial y}+\frac{\varepsilon}{2} \frac{\partial^{2} v}{\partial y^{2}}+\frac{\varepsilon^{\prime}}{2} \frac{\partial^{2} v}{\partial x^{2}}=0 .
$$

Lemma A.6. Assume $b(\cdot, \cdot)$ satisfies (1.1) and let $v(x, y, t)$ for $(x, y) \in D_{R}$ and $t<T$, be a solution of (A.34) which is $C^{2}$ in $(x, y)$ and $C^{1}$ in $t$. Assume further that $v(x, y, t)$ extends to a continuous function on $\bar{D}_{R} \times\{t \leq T\}$. Let $w(x, y, t)$ be a second solution to (A.34) with similar properties to those of $v(x, y, t)$. Then if for some constant $M$ the inequality

$$
\int_{t}^{T}|b(0, s)| d s+A(T-t)+\sqrt{\varepsilon(T-t)} \leq M
$$

holds, there is a constant $C$ depending only on $M$ such that

$$
\begin{aligned}
& \text { (A.41) }|v(0,0, t)-w(0,0, t)| \\
& \quad \leq \exp \left[-\frac{R^{2}}{C \varepsilon(T-t)}\right] \sup \left\{|v(x, y, s)-w(x, y, s)|: t \leq s<T, \quad(x, y) \in \partial D_{R}\right\} \\
& \quad+\sum_{k \geq 0} \exp \left[-\frac{k^{2}}{C \varepsilon(T-t)}\right] \sup \left\{|v(x, y, T)-w(x, y, T)|:(x, y) \in D_{k+M} \cap D_{R}\right\},
\end{aligned}
$$

provided $0<\varepsilon^{\prime} \leq \varepsilon$. 
Proof. We set $u(x, y, t)=v(x, y, t)-w(x, y, t)$, and observe from (A.34) that $u(x, y, t)$ satisfies the differential inequality

$$
\frac{\partial u}{\partial t}-|b(y, t)|\left|\frac{\partial u}{\partial y}\right|+\frac{\varepsilon}{2} \frac{\partial^{2} u}{\partial y^{2}}+\frac{\varepsilon^{\prime}}{2} \frac{\partial^{2} u}{\partial x^{2}} \leq 0 .
$$

Suppose now that $C(x, y, t)$ satisfies

$$
\frac{\partial C}{\partial t}-|b(y, t)|\left|\frac{\partial C}{\partial y}\right|+\frac{\varepsilon}{2} \frac{\partial^{2} C}{\partial y^{2}}+\frac{\varepsilon^{\prime}}{2} \frac{\partial^{2} C}{\partial x^{2}}=0, \quad(x, y) \in D_{R}, t<T
$$

with boundary and terminal data given by

$$
\begin{aligned}
& C(x, y, T)=u(x, y, T), \quad(x, y) \in D_{R} ; \\
& C(x, y, t)=u(x, y, t), \quad(x, y) \in \partial D_{R}, t<T .
\end{aligned}
$$

Then by the maximum principle we have that $u(x, y, t) \geq C(x, y, t)$ for $(x, y) \in$ $D_{R}$ and $t<T$. Observe next that $C(x, y, t)$ is the cost function for an optimal control problem. Thus

$$
\begin{aligned}
C(x, y, t)=\inf _{\lambda(\cdot, \cdot)}\{E[ & \left.u(X(T), Y(T), T) ; \tau_{x, y, t}>T\right] \\
& \left.+E\left[u\left(X\left(\tau_{x, y, t}\right), Y\left(\tau_{x, y, t}\right), t\right) ; \tau_{x, y, t}<T\right]\right\},
\end{aligned}
$$

where the stochastic process $[X(s), Y(s)]$ satisfies the SDE

$$
d Y(s)=\lambda(Y(s), s) d s+\sqrt{\varepsilon} d W(s), \quad d X(s)=\sqrt{\varepsilon^{\prime}} d W^{\prime}(s),
$$

and $W(\cdot), W^{\prime}(\cdot)$ are independent copies of Brownian motion. The controller $\lambda(y, s)$ satisfies the constraints $|\lambda(y, s)| \leq|b(y, s)|$ for $y \in \mathbb{R}$ and $s \leq T$. The stopping time $\tau_{x, y, t}$ is the first hitting time on $\partial D_{R}$ for the process (A.46) with $X(t)=x$ and $Y(t)=y$.

If we argue now as we did in Lemma 3.1 we can see that $C(0,0, t)$ is bounded below by the negative of the right-hand side of (A.41). Thus we obtain a lower bound on $v(0,0, t)-w(0,0, t)$. Since we can repeat the previous argument with $v$ and $w$ interchanged, we also get an upper bound on $v(0,0, t)-w(0,0, t)$, whence (A.41) follows.

Proposition A.7. Assume $b(\cdot, \cdot)$ satisfies (1.1), and the terminal function $v_{0}(x, y)$ in Proposition A.4 is logarithmically concave and satisfies the boundary condition $\left|D v_{0}(x, y)\right| \neq 0$ for $(x, y) \in \partial D_{R}$. If in addition the function $b(y, t)$ is concave in $y$ for $y \in \mathbb{R}, t \leq T$, then the solution $v(x, y, t)$ of (A.34) and (A.35) is also logarithmically concave.

Proof. We again follow the method of Korevaar [14] as given in [9] (see also [10]). Thus on setting $w(x, y, t)=-\log v(x, y, t)$ we see from (A.34) that $w(x, y, t)$ satisfies the PDE

$$
\frac{\partial w}{\partial t}+\frac{\varepsilon}{2} \frac{\partial^{2} w}{\partial y^{2}}+\frac{\varepsilon^{\prime}}{2} \frac{\partial^{2} w}{\partial x^{2}}-B(y, t, D w)=0
$$


where the function $B(y, t, p)$ is given by the formula

$$
B(y, t, p)=b(y, t)\left|p_{y}\right|+\varepsilon p_{y}^{2} / 2+\varepsilon^{\prime} p_{x}^{2} / 2
$$

Since $B(y, t, p)$ satisfies the conditions of Theorem 4.1 of [9], the result follows provided we can show that $w(x, y, t)$ is convex for $(x, y, t)$ close to the boundary of $D_{R} \times\{t<T\}$. To see this we argue as in Lemma 2.4 of [14]. Observe that it is sufficient to assume $D^{2} v(x, y, t)$ is bounded as in (A.36), and not necessarily continuous as $(x, y, t)$ approaches a boundary point, provided the tangential derivative of $D v(x, y, t)$ remains continuous. To see why this is the case consider a nonnegative $C^{2}$ function $f$ on the half plane $H=\left\{(x, z) \in \mathbb{R}^{2}: z>0\right\}$. We assume that $f$ extends to a $C^{1}$ function on the closure $\bar{H}$ of $H$ and that $f \equiv 0$ on $\partial H$. In addition we assume the boundary behavior at $(0,0)$ of the second derivatives of $f$ is given by

$\limsup _{(x, z) \rightarrow(0,0)}\left|\frac{\partial^{2} f}{\partial x \partial z}(x, z)\right|<\infty, \lim _{(x, z) \rightarrow(0,0)} \frac{\partial^{2} f}{\partial x^{2}}(x, z)=0, \limsup _{(x, z) \rightarrow(0,0)}\left|\frac{\partial^{2} f}{\partial z^{2}}(x, z)\right|<\infty$.

Now define a function $w(x, y)$ on the domain $U=\left\{(x, y) \in \mathbb{R}^{2}: y>x^{2} / 2\right\}$ by $\exp [-w(x, y)]=f\left(x, y-x^{2} / 2\right)$. Then we can see that if $\partial f(0,0) / \partial z>0$, there exists $\delta>0$ such that the Hessian of $w$ is strictly positive definite for $(x, y) \in$ $U \cap D_{\delta}$. The convexity of $w(x, y, t)$ close to the boundary of $D_{R} \times\{t<T\}$ follows from the regularity result Proposition A.4 and Lemma A.5 by an analogous argument.

Theorem A.8. Assume $b(\cdot, \cdot)$ satisfies (1.1) and in addition the function $b(y, t)$ is concave in $y$ for $y \in \mathbb{R}$ and $t \leq T$. Then for $t<T$ the function $q_{\varepsilon}(x, y, t)$ is convex in $(x, y)$ for $(x, y) \in \mathbb{R}^{2}$.

Proof. Similarly to the proof of Theorem A.2, we approximate $q_{\varepsilon}(x, y, t)$ by functions defined on finite domains $D_{R}$ which are convex by virtue of Proposition A.7. To specify the terminal function $v_{0}(x, y)$, we define a function $f(z)$ for $z<1$ by

$$
\begin{aligned}
f(z) & =0 \quad \text { for } z<1 / 2, \quad f(1 / 2)=f^{\prime}(1 / 2)=0 \\
f^{\prime \prime}(z) & =\frac{\exp \left[-(1-z)^{2} /(2 z-1)\right]}{(1-z)^{2}}, \quad \text { for } 1 / 2<z<1 .
\end{aligned}
$$

Evidently $f(\cdot)$ is a nonnegative increasing $C^{\infty}$ convex function which has the property that $f(z)+\log (1-z)$ has a Taylor expansion about $z=1$ with positive radius of convergence. Next let $g: \mathbb{R} \rightarrow \mathbb{R}$ be defined by

$$
g(z)=z^{4}, \quad z<0 ; \quad g(z)=0, \quad z \geq 0
$$

whence $g$ is a nonnegative decreasing $C^{3}$ convex function. It follows from (A.50) and (A.51) that the function $v_{0}$ with domain $D_{R}$ defined by

$$
v_{0}(x, y)=\exp \left[-f\left(\sqrt{x^{2}+y^{2}} / R\right)-g([y-x] / \delta)\right]
$$


is $C^{2}$ for $(x, y) \in \bar{D}_{R}$ with $v_{0}(x, y)=0$ if $(x, y) \in \partial D_{R}$. In addition $v_{0}(x, y)$ is logarithmically concave for $(x, y) \in D_{R}$ and satisfies the nondegenerate boundary condition $\left|D v_{0}(x, y)\right| \neq 0$ if $(x, y) \in \partial D_{R}$. Hence by Proposition A.7 the corresponding solution $v_{\delta, R}(x, y, t)$ of (A.34) and (A.35) is logarithmically concave in $(x, y)$.

Next we compare the function $v_{\delta, R}(x, y, t)$ to a solution of the linear equation (A.39). Thus let $v_{\delta}(x, y, t)$ be the unique bounded solution to (A.39) in the domain $\left\{(x, y, t):(x, y) \in \mathbb{R}^{2}, t<T\right\}$ with terminal condition

$$
v_{\delta}(x, y, t)=\exp [-g([y-x] / \delta)], \quad(x, y) \in \mathbb{R}^{2} .
$$

From (A.51) one sees that $v_{\delta}(x, y, T)$ is an increasing function of $y$ for every $x \in \mathbb{R}$. The maximum principle implies then that $v_{\delta}(x, y, t)$ is also an increasing function of $y$ for every $x \in \mathbb{R}$ and $t<T$. Thus $v_{\delta}(x, y, t)$ is also a solution to (A.34). We may therefore use Lemma A. 6 to compare the functions $v_{\delta}$ and $v_{\delta, R}$. In view of the fact that $0 \leq v_{\delta} \leq 1$ and the properties of the function $f$ of (A.50), we conclude from (A.52) and (A.53) that

(A.54) $\limsup _{R \rightarrow \infty}\left\{\left|v_{\delta}(x, y, t)-v_{\delta, R}(x, y, t)\right|:(x, y) \in D_{R_{0}}, T_{0} \leq t<T\right\}=0$,

for any $R_{0}>0$ and $T_{0}<T$.

We conclude from (A.54) and the logarithmic concavity of $v_{\delta, R}$ that the function $v_{\delta}(x, y, t)$ is also logarithmically concave in $(x, y)$ for $(x, y) \in \mathbb{R}^{2}$ and $t<T$. Observe here that we are using the strong maximum principle to conclude that $v_{\delta}(x, y, t)>0$ for $(x, y) \in \mathbb{R}^{2}$ and $t<T$. Next we see that the function $v(x, y, t)=$ $\lim _{\delta \rightarrow 0} v_{\delta}(x, y, t)$ is the unique bounded solution of (A.39) which has terminal data $v(x, y, T)=0$ if $y<x, v(x, y, T)=1$ if $y>x$. Thus $v(x, y, t)=v_{\varepsilon, \varepsilon^{\prime}}(x, y, t)$ is logarithmically concave for $(x, y) \in \mathbb{R}^{2}$ and $t<T$. Finally we conclude the convexity of $q_{\varepsilon}(x, y, t)$ in $(x, y)$ by noting that the function $u_{\varepsilon}(x, y, t)$ of $(1.2)$ and $(1.3)$ satisfies $u_{\varepsilon}(x, y, t)=\lim _{\varepsilon^{\prime} \rightarrow 0} v_{\varepsilon, \varepsilon^{\prime}}(x, y, t)$.

\section{References}

[1] Alvarez, O., Lasry, J. M. and Lions, P. L. : Convex viscosity solutions and state constraints. J. Math. Pures Appl. (9) 76 (1997), no. 3, 265-288.

[2] Crandall, M. And Lions, P. L.: Two approximations of solutions of HamiltonJacobi equations. Math. Comp. 43 (1984), no. 167, 1-19.

[3] Evans, L. C.: Adjoint and compensated compactness methods for Hamilton-Jacobi PDE. Arch. Ration. Mech. Anal. 197 (2010), no. 3, 1053-1088.

[4] Fleming, W.: The convergence problem for differential games. II. In Advances in Game Theory, 195-210. Princeton University Press, Princeton, NJ, 1964.

[5] Fleming, W. And Rishel, R.: Deterministic and stochastic optimal control. Applications of Mathematics 1, Springer-Verlag, Berlin-New York, 1975.

[6] Fleming, W. And Sheu, S.: Stochastic variational formula for fundamental solutions of parabolic PDE. Appl. Math. Optim. 13 (1985), no. 3, 193-204. 
[7] Friedman, A.: Partial differential equations of parabolic type. Prentice-Hall, Englewood Cliffs, NJ, 1964.

[8] Freidlin, M. And Wentzell, A. D.: Random perturbations of dynamical systems. Grundlehren der Mathematischen Wissenschaften 260, Springer, Heidelberg 2012.

[9] Greco, A. And Kawohl, B.: Log-concavity in some parabolic problems. Electron. J. Differential Equations 19 (1999), 12 pp.

[10] Greco, A. And Porru, G.: Convexity of solutions to some elliptic partial differential equations. SIAM J. Math. Anal. 24 (1993), no. 4, 833-839.

[11] KannaI, Y.: Off diagonal short time asymptotics for fundamental solutions of diffusion equations. Commun. Partial Differ. Equations 2 (1977), no. 8, 781-830.

[12] Karatzas, I. And Shreve, S.: Brownian motion and stochastic calculus. Graduate Texts in Mathematics 113, Springer-Verlag, New York, 1991.

[13] KIfER, Y.: On the asymptotic behavior of transition density of processes with small diffusion. Theory of Probability and its Applications 21 (1976), no. 3, 527-536.

[14] KorevaAr, N.: Convex solutions to nonlinear elliptic and parabolic boundary value problems. Indiana Univ. Math. J. 32 (1983), no. 4, 603-614.

[15] Lieberman, G.: Second order parabolic differential equations. World Scientific, River Edge, NJ, 1996.

[16] Lions, P. L. And Musiela, M.: Convexity of solutions of parabolic equations. C. $R$. Math. Acad. Sci. Paris 342 (2006), no. 12, 915-921.

[17] Minakshisundaram, S.: Eigenfunctions on Riemannian manifolds. J. Indian Math. Soc. (N.S.) 17 (1953), 159-165.

[18] Molchanov, S.: Diffusion processes and Riemannian geometry. Uspehi Mat. Nauk. 30 (1975), no. 1, 3-59.

[19] Protter, M. And Weinberger, H.: Maximum principles in differential equations. Springer-Verlag, New York, 1984.

[20] Reed, M. And Simon, B.: Methods of modern mathematical physics. I. Functional analysis. Academic Press, New York-London, 1972.

[21] Simon, B.: Functional integration and quantum physics. AMS Chelsea Publishing, Providence, RI, 2005.

[22] Varadhan, S. R. S.: On the behavior of the fundamental solution of the heat equation with variable coefficients. Comm. Pure Appl. Math. 20 (1967), 431-455.

Received July 11, 2012.

Joseph G. Conlon: University of Michigan, Department of Mathematics, Ann Arbor, MI 48109-1109, USA.

E-mail: conlon@umich.edu

Mohar Guha: University of Michigan, Department of Mathematics, Ann Arbor, MI 48109-1109, USA.

E-mail: mguha@umich.edu

This research was partially supported by the U.S. National Science Foundation under grants DMS-0500608 and DMS-0553487. 